Florida International University FIU Digital Commons

8-4-2005

\title{
The Study of Sonar for Imaging of the Solid-Liquid Interface Inside Large Tanks
}

Nitin Sood

Florida International University, nitin0sood@hotmail.com

DOI: $10.25148 /$ etd.FI08081547

Follow this and additional works at: https://digitalcommons.fiu.edu/etd

\section{Recommended Citation}

Sood, Nitin, "The Study of Sonar for Imaging of the Solid-Liquid Interface Inside Large Tanks" (2005). FIU Electronic Theses and Dissertations. 30.

https://digitalcommons.fiu.edu/etd/30 


\section{FLORIDA INTERNATIONAL UNIVERSITY \\ Miami, Florida}

\section{STUDY OF SONAR FOR IMAGING \\ OF THE SOLID-LIQUID INTERFACE INSIDE LARGE TANK}

A thesis submitted in partial fulfillment of the

requirements for the degree of

MASTER OF SCIENCE

in

COMPUTER ENGINEERING

by

Nitin Sood

2005 
To: Dean Vish Prasad

College of Engineering and Computing

This thesis, written by Nitin Sood, and entitled, Study of Sonar for Imaging of the SolidLiquid Interface Inside Large Tank, having been approved in respect to style and intellectual content, is referred to you for judgment.

We have read this thesis and recommend that it be approved.

David Roelant

Rajiv Srivastava

Kang Yen, Major Professor

Date of Defense: August 4, 2005

The thesis of Nitin Sood is approved.

Dean Vish Prasad College of Engineering and Computing

Dean Douglas Wartzok

University Graduate School

Florida International University, 2005 


\section{DEDICATION}

I dedicate this thesis to my parents Vinod Kumar Sood and Neelam Sood, and to my brother, Nikhil, who encouraged and supported me. Without their patience, understanding, support and most of all love, the completion of this work would not have been possible. 


\section{ACKNOWLEDGMENTS}

I wish to express my sincerest thanks to Dr. David Roelant who provided the research opportunity, intellectual and financial support; served as my daily advisor; and constantly encouraged me when experimental and computational difficulties arose. I would like to express my deepest appreciation to Dr. Kang Yen for his insight, constant support, and guidance throughout my graduate study. I thank Dr. Rajiv Srivastava for his patience advice and assistance in writing this thesis. I also thank the Hemispheric Center for Environmental Technology (HCET) organization for providing staff expertise when needed and top quality equipment and facilities for my research.

Finally, I would like to thank my friends, peers, and fellow students who were always there to answer my questions, help bring clarification to difficult problems, and provide relief. 


\begin{abstract}
OF THE THESIS
STUDY OF SONAR FOR IMAGING OF THE SOLID-LIQUID INTERFACE INSIDE

LARGE TANK

by

Nitin Sood
\end{abstract}

Florida International University, 2005

Miami, Florida

Professor Kang Yen, Major Professor

Retrieval, treatment, and disposal of high-level radioactive waste (HLW) are expected to cost between 100 and 300 billion dollars. The risk to workers, public health, and the environment are also a major area of concern for HLW. Visualization of the interface between settled solids and the optically opaque liquid is needed for retrieval of the waste from underground storage tanks. The profiling sonar selected for this research generates 2-D image of the interface. Multiple experiments were performed to demonstrate the effectiveness of sonar in real-time monitoring of the interface inside HLW tanks. Initial experiments demonstrated that various objects shapes could be identified even when $30 \%$ of solids were entrained in liquid. Simulations of the sonar system validated these results. The second set of experiments confirmed the sonar's ability to detect a solid interface with density similar to the liquid. The third set of experiments determined the effects of nearby objects on image resolution. The final set of experiments demonstrated functionality and chemical capability of the sonar in highly caustic solutions. 


\section{TABLE OF CONTENTS}

CHAPTER

PAGE

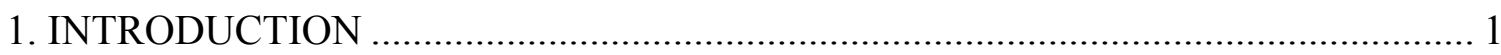

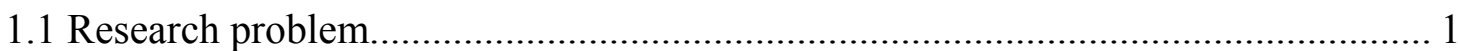

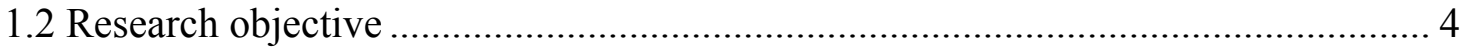

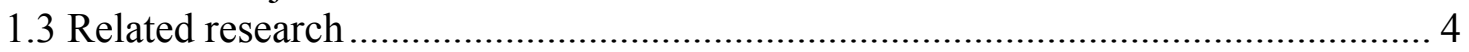

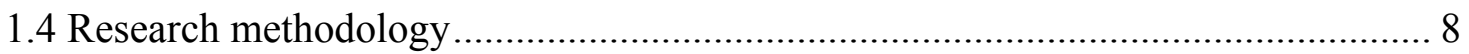

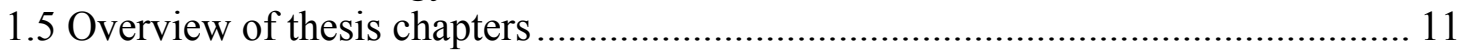

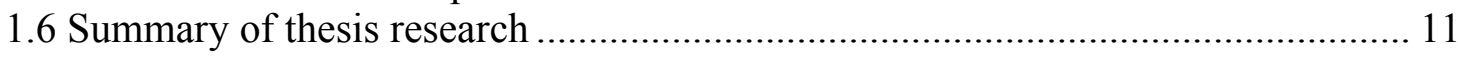

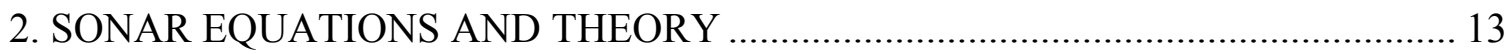

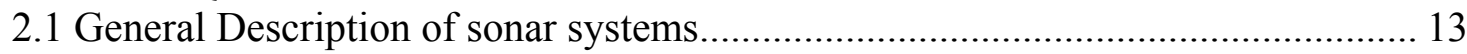

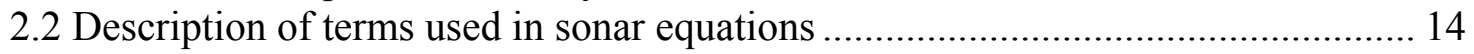

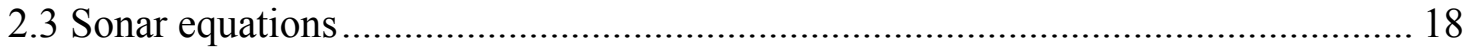

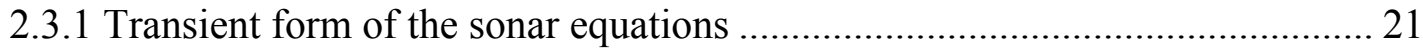

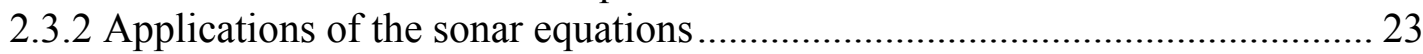

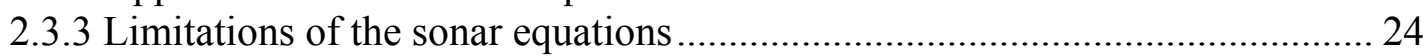

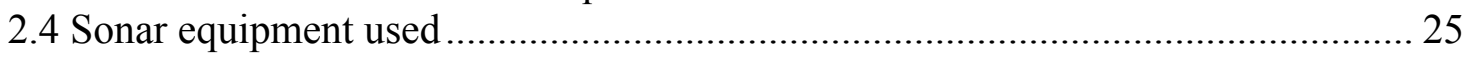

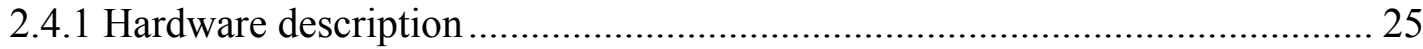

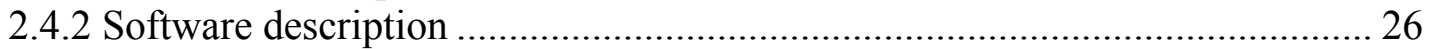

2.5 Calculations of transmission loss for Imagenex 881A profiling sonar.................... 27

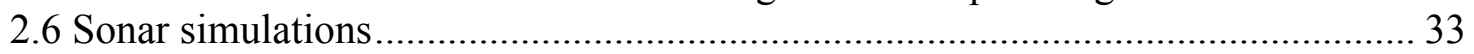

3. EXPERIMENTAL RESEARCH AND RESULTS ....................................................42

3.1. Summary of the seven sets of experiments conducted ....................................... 42

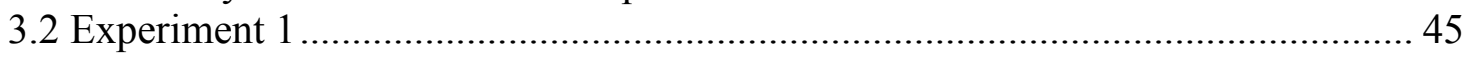

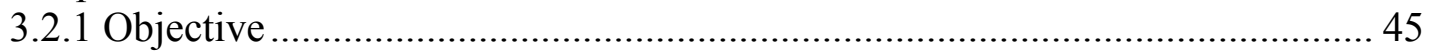

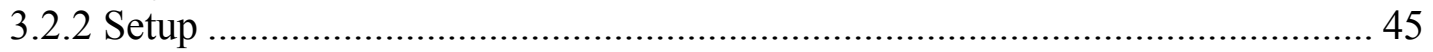

3.2.3 Detection of metal drum ........................................................................... 45

3.2.4 Detection of metal objects ............................................................................. 47

3.2.5 Detection of the solids layer gradient inside a tank ...................................... 48

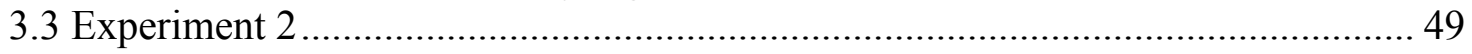

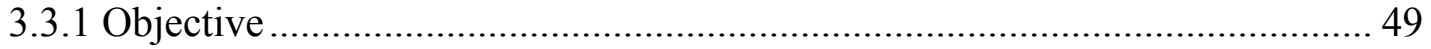

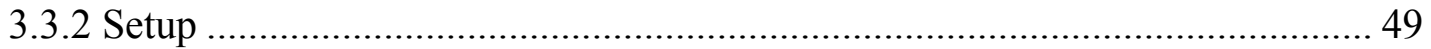

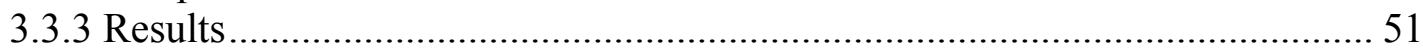

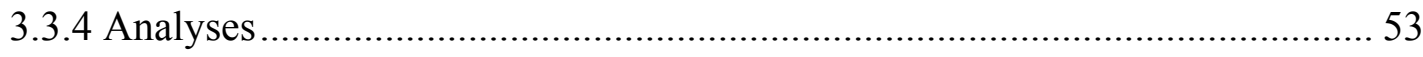

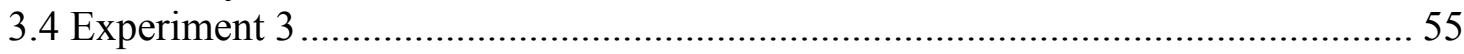

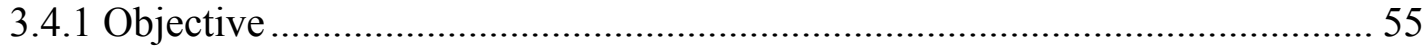

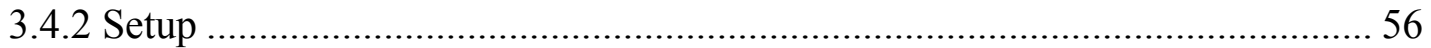

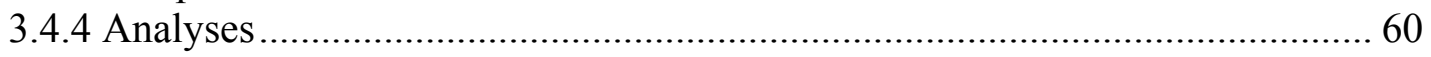

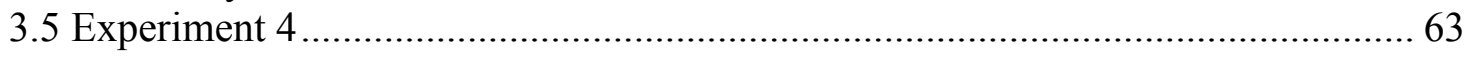

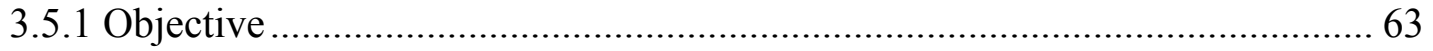

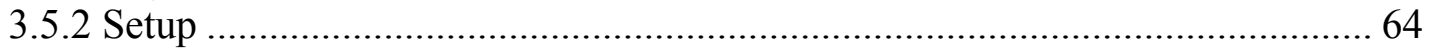




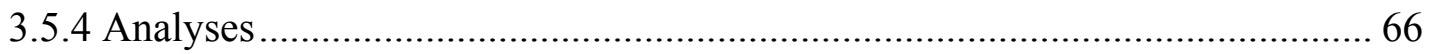

3.6 Experiment 5

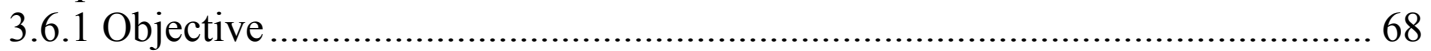

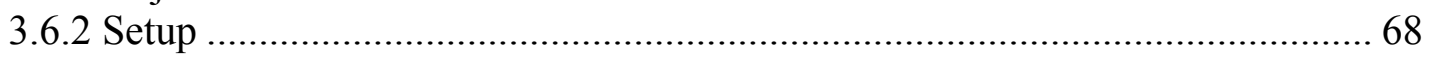

3.6.3 Effect of a vertical metal plate placed near the sonar head............................... 69

3.6.4 Effect of sonar placed near a horizontal solids layer ...................................... 71

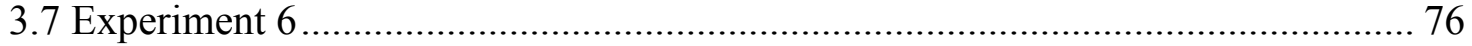

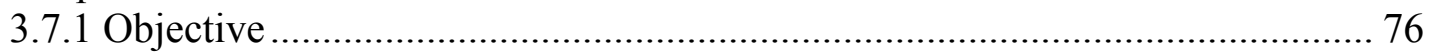

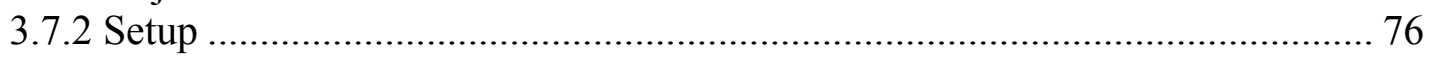

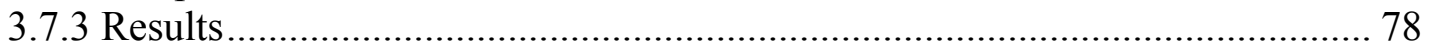

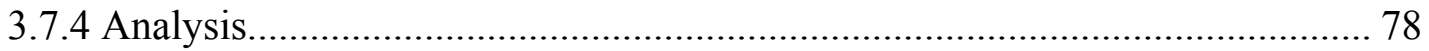

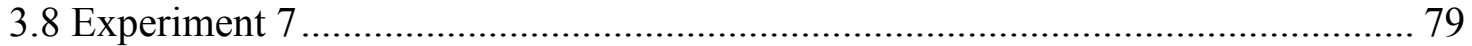

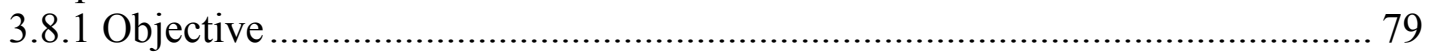

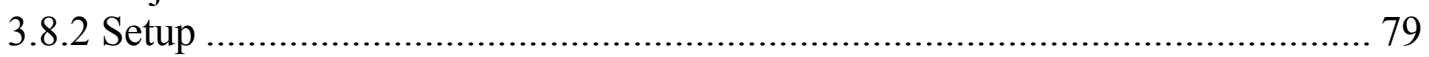

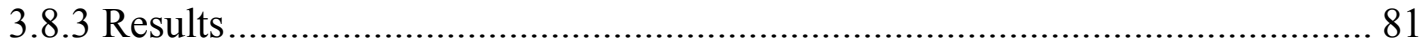

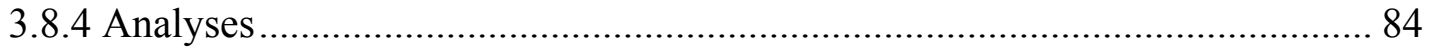

4. CONCLUSIONS AND POTENTIAL FUTURE WORK …........................................... 90

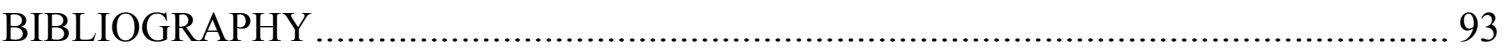

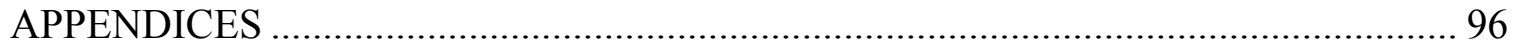




\section{LIST OF TABLES}

TABLE

PAGE

1. Sonar Parameters, their Definitions, and Reference Locations ..................................... 17

2. Terminology of Various Combinations of the Sonar Parameters .................................. 20

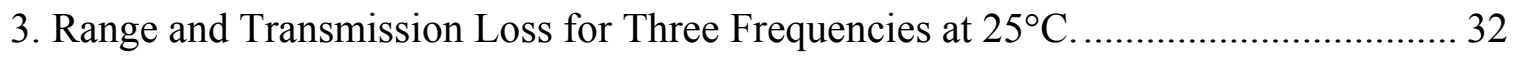

4. Actual and Observed Measurements of the Interface and Sonar Heights. ................... 53

5. Actual and Observed Values of the Interface Height Before and During Agitation. ... 61

6. Actual and Observed Measurements of the Metal Object with Relative Error............ 62

7. Actual and Observed Locations and Dimensions of Different Metal Forms Inside a Tank.

8. Actual and Observed Measurements (with Relative Error) of Metal Plate. 70

9. Actual and Observed Measurements (with Relative Error) of Distances between the Bricks.

10. Actual and Observed Measurements (with Relative Error) of Tank.

11. Actual and Observed Measurements (with Relative Error) of Distance between the Bricks.

12. Actual and Observed Measurements (with Relative Error) of Brick Heights. 75

13. Actual and Observed Dimensions of the Objects with its Relative Error. 79

14. Actual, Observed, and Relative Error in Heights at Three Temperatures at Default Sound Speed.

15. Actual, Observed and Relative Error in Heights at Corrected Sound Speed 86

16. Actual, Observed, and Relative Error in Heights for Solutions of Different Densities at $25^{\circ} \mathrm{C}$ with Default and Corrected Sound Speed.

17. Actual, Observed, and Relative Error in Heights for Solutions of Different Densities at $30^{\circ} \mathrm{C}$ with Default and Corrected Sound Speed. 


\section{LIST OF FIGURES}

FIGURE

PAGE

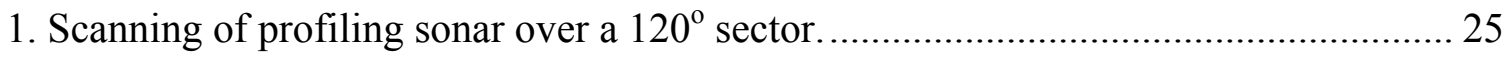

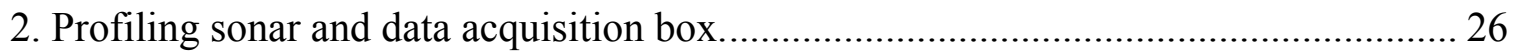

3. Image displaying the output of sonar with two-cursor measurement and pixel

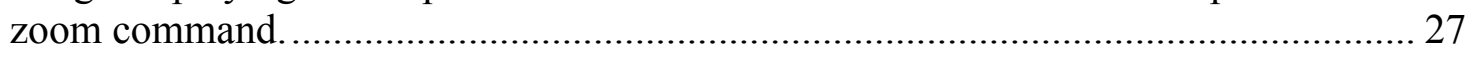

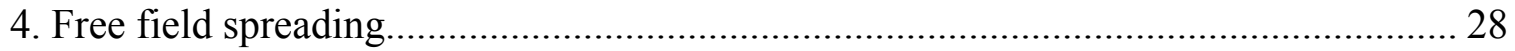

5. Transmission loss over a specified range at $25^{\circ} \mathrm{C}$ for three frequencies. ..................... 33

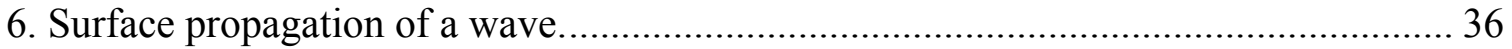

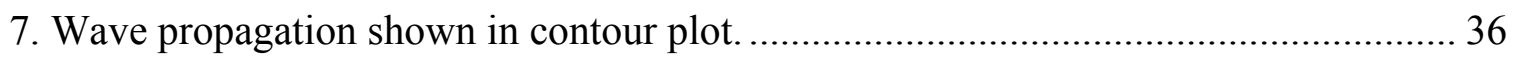

8. Enlarged image of wave contour plotalso showing the conical shape of the wave...... 37

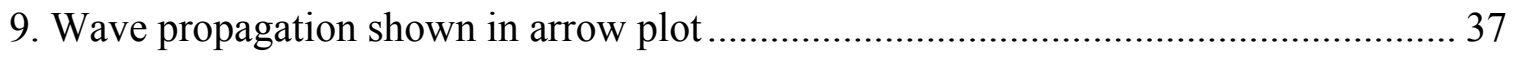

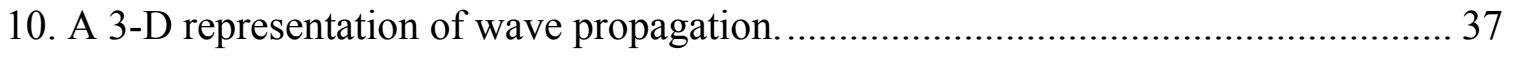

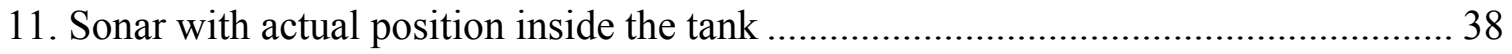

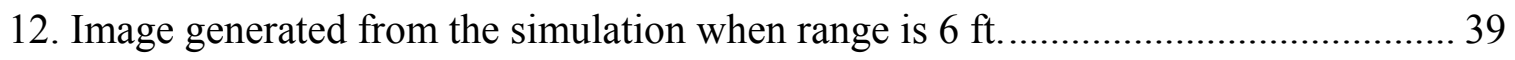

13. Propagation of a single wave incident at $67^{\circ}$ from the transducer and the range is

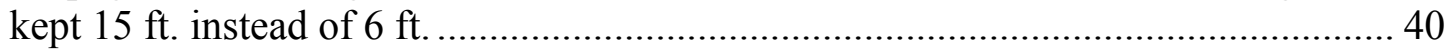

14. Image generated from simulation when range was kept $15 \mathrm{ft}$............................... 40

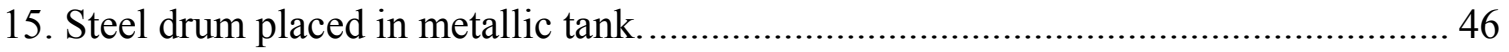

16. Image of the drum generated by the sonar when it is placed vertically in the tank... 46

17. Image of the drum when it is placed horizontally in the tank .............................. 46

18. A square metal plate with metallic object placed on top of it............................... 47

19. Image of the metal plate with a metallic object on top of it. ................................... 47

20. The gradient formed by kaolin clay inside the tank......................................... 48 
21. Gradient seen by sonar.

22. Top view of the interface created by kaolin clay and tank bottom 49

23. Side view of the tank showing the interface. 49

24. Cross section of the tank. 50

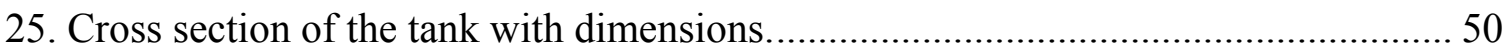

26. Different positions of the sonar where interface was imaged. 51

27. Images of interface generated by sonar software when sonar is suspended at different positions in the tank.

28. Bar plot of actual and observed values of the height of sonar from tank bottom at 9 different positions.

29. Bar plot of actual and observed values of the interface height when sonar is placed at 9 different positions

30. Plot of relative error in the sonar and interface height when sonar is placed at 9 different positions. 55

31. Top view of the tank showing the interface, the metal object in the tank. 56

32. Interface generated when sonar is placed at position 1, (a) no agitation, and (b) during agitation.

33. Interface generated when sonar is placed at position 2, (a) no agitation, and (b) during agitation.

34. Image of the interface when sonar is placed at position 3 (a) no agitation, and (b) during agitation.

35. Interface generated when sonar is placed at position 4, (a) no agitation, and (b) during agitation. 58

36. Interface generated when sonar is placed at position 5, (a) no agitation, and (b) during agitation. 58

37. Interface generated when sonar is placed at position 6 , (a) no agitation, and (b) during agitation. 
38. Interface generated when sonar is placed at position 7, (a) no agitation, and (b) during agitation

39. Interface generated when sonar is placed at position 8 , (a) no agitation, and (b) during agitation.

40. Interface generated when sonar is placed at position 9, (a) no agitation, and (b) during agitation.

41. Bar plot of the actual and observed values of the distance between the tank wall and the interface.

42. Bar plot of actual and the observed distance between the different pipes of the metal object when the sonar is at position 9 .

43. Top view of the tank with diagonal I-beam. Plastic beads in and around 3 metal forms (left) and in 2 metal forms (right). Sonar suspended from the U-channel

44. Top view of the tank showing the placement of the geometric forms ("a", "b", "c", "d", "e" and "f) and image plane of the sonar.

45. Side view of the sonar scanning plane

46. Image generated when plastic beads are placed inside the tank. "b", "c","d" and "e" are the objects as shown in Figures 43 and 44. 66

47. Top view of the tank with bricks placed inside it. 68

48. Cross section of the tank with the placement of the bricks and the sonar. 69

49. Cross section of the tank with metal plate placed in it at $1.5 \mathrm{ft}$. from sonar head...... 69

50. Image generated when metal plate is placed $10 \mathrm{in}$. from sonar................................. 69

51. Image generated when metal plate is placed 13 in. from sonar................................. 69

52. Image generated when metal plate is placed 14 in. from sonar................................ 69

53. Image generated when metal plate is placed 16 in. from sonar................................. 69

54. Plot of relative errors in measurements of distance between bricks when metal

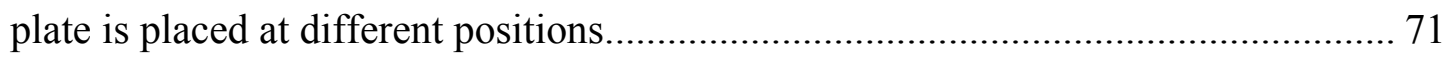

55. Image generated when sonar head is suspended $2 \mathrm{ft}$. from tank bottom. 71 
56. Image generated when sonar head is suspended $1.5 \mathrm{ft}$. from tank bottom 71

57. Image generated when sonar head is suspended $1 \mathrm{ft}$. from tank bottom. 72

58. Image generated when sonar head is suspended $0.8 \mathrm{ft}$. from tank bottom. 72

59. Image generated when sonar head is suspended $0.6 \mathrm{ft}$. from tank bottom. 72

60. Image generated when sonar head is suspended $0.5 \mathrm{ft}$. from tank bottom 72

61. Relative error in the measurements of the distance between the bricks when sonar is placed at different heights from tank bottom 74

62. Top view of the drum with sonar placed in caustic solution. 77

63. The drum with tape heater wound around it (left) and the placement of sonar inside the drum (right).

64. Cross-section of the steel drum, with bricks and sonar. 77

65. Aluminum band destroyed by caustic solution 78

66. Image obtained by sonar for the arrangement shown in Figure 64 78

67. Top view of the drum with two stainless steel objects placed on U-channel stand.... 80

68. Cross-section of the steel drum, with metal pieces and sonar. 80

69. Placement of mixer in the drum filled with sodium nitrate solution 81

70. Images generated when water is temperature is heated to $25^{\circ} \mathrm{C}(\mathrm{a}), 30^{\circ} \mathrm{C}(\mathrm{b})$, and $35^{\circ} \mathrm{C}(\mathrm{c})$

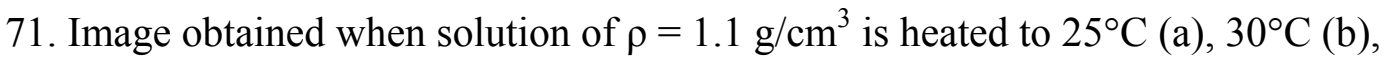
and $35^{\circ} \mathrm{C}(\mathrm{c})$

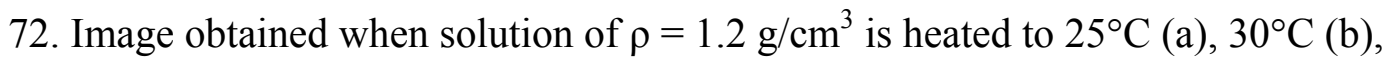
and $35^{\circ} \mathrm{C}(\mathrm{c})$

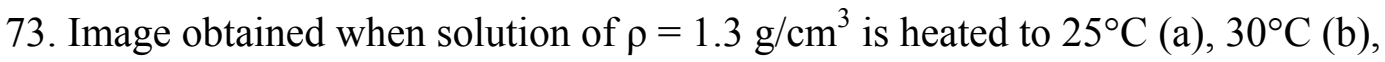
and $35^{\circ} \mathrm{C}(\mathrm{c})$

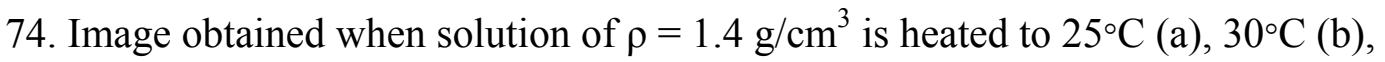
and $35^{\circ} \mathrm{C}(\mathrm{c})$. 
75. Screen shot of the software to generate the correct sound speed for different

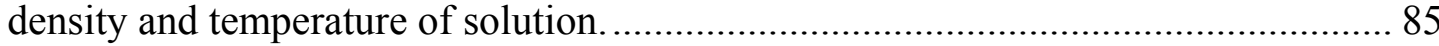

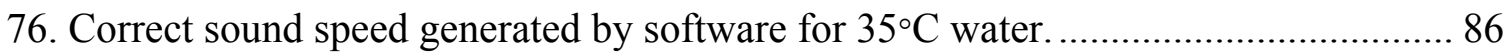

77. Plot of change in sound speed at different temperature when density of the water is increased from $0.99 \mathrm{~g} / \mathrm{cm}^{3}$ to $1.4 \mathrm{~g} / \mathrm{cm}^{3}$. 


\section{Glossary}

\begin{tabular}{|c|c|}
\hline$\alpha$ & Attenuation coefficient \\
\hline$\rho$ & Density \\
\hline DI & Directive Index \\
\hline $\mathrm{DI}_{\mathrm{S}}$ & Directivity of Target-Source \\
\hline $\mathrm{DI}_{\mathrm{T}}$ & Directivity of Target \\
\hline DT & Detection Threshold \\
\hline $\mathrm{E}$ & Energy \\
\hline $\mathrm{f}$ & Frequency \\
\hline FIU & Florida International University \\
\hline HCET & Hemispheric Center for Environmental Technology \\
\hline HLW & High-Level Radioactive Waste \\
\hline $\mathrm{I}$ & Intensity \\
\hline NL & Noise Level \\
\hline $\mathrm{P}$ & Pressure \\
\hline $\mathrm{R}$ & Range \\
\hline RL & Reverberation Level \\
\hline $\mathrm{S}$ & Salinity \\
\hline SL & Source Level \\
\hline $\mathrm{T}$ & Time \\
\hline $\mathrm{TL}$ & Transmission Loss \\
\hline TS & Target Strength \\
\hline
\end{tabular}




\section{Chapter 1}

\section{INTRODUCTION}

\subsection{Research problem}

Disposal of radioactive hazardous waste is an area of major concern. At present most of the waste generated during the production of nuclear weapons, a legacy of the cold war era, is stored in large underground storage tanks at three major United States Department of Energy (DOE) facilities. The high-level radioactive waste (HLW) inside these tanks are generally kept at a $\mathrm{pH}>14$ in order to minimize the corrosion of the tanks. The radioactivity inside these HLW tanks elevates the temperature to about $30-40^{\circ} \mathrm{C}$.

The DOE Hanford site has the largest number of HLW storage tanks and the largest volume of HLW in the United States. At present there are 269 HLW tanks at the Hanford site alone with some tanks possessing a capacity greater than one million gallons. The retrieval and treatment for safe disposal of approximately 55 million gallons of HLW stored in Hanford's underground tanks poses a considerable challenge as 32 tanks have had confirmed leakage. This has resulted a risk to workers, public health and environment as the Columbia River is 5-10 miles from these tanks. Removal from the tank, treatment, and disposal of high-level waste, constitutes a lasting solution to this challenging problem. The total unused volume in these tanks is small making it difficult to empty a tank without moving waste to multiple tanks or sending HLW to an evaporator in order to reduce the volume.

The traditional method for locating the level of solids in underground storage tanks is to lower a weighted ring into the tank and measure the level at a single point. 
This method is inaccurate as it measures only at single point in 70 -feet diameter tank, secondly, the ring may sink into low density solids, thus forming a depression in the solids surface with repeated measurements. Upon retrieval, the waste is often pumped through 2-inch pipes for distances ranging from hundreds of feet to miles. Lower temperatures within these transfer pipelines, and the placement of the retrieval pump to close to the solids layer in the past have led to plugged lines, with costs exceeding $\$ 3 \mathrm{M}$ to unplug or install a single pipeline. Due to the aggressive schedule for treatment of HLW over the next decade, the DOE site engineers have identified a critical need for a solidliquid interface monitor inside HLW tanks to maximize the amount of solids transferred into a given tank without risking the plugging of the transfer pipelines.

The Hanford site has set the following minimum criteria for a monitor for mapping the solid-liquid interface: 1) Deployable through a 4-inch access pipes, 2) operable in extremely caustic solution and in high nuclear radiation exposures, and 3) able to map the settled solids layer over an area of at least 5 square feet. Researchers form Florida International University worked together with Hanford site engineers and imaging experts from around the country to assess optimal monitoring technology solutions.

Imaging using an active, interrogating source from across the entire electromagnetic spectrum from gamma rays to the radio waves were eliminated based upon physical principles and lack of contrast between liquid and settled solids using these sources. Electrical and acoustic imaging methods were identified as the only ones to be able to image the settled solids layer. 
Of the many electrical imaging methods (one not based on photon or waves) only one has the ability to image a solids layer inside a conducting liquid by placing sensors (electrodes) at the periphery of the selected volume. This method ERT or EIT was the focus of a similar research effort as this.

There were many acoustic imaging methods based upon acoustic frequency and beam forming processes the profiling sonar was identified as the best method for obtaining the best resolution of the settled solids layer. The specific model of profiling sonar was one manufactured by Imagenex Inc. due to its higher resolution imaging over short distances for sonars (i.e., 1-50 ft.).

Reasons for selecting sonar

Factors that lead to the selection of sonar are following:

1. Sonar is water based system. It has been proven to operate efficiently in underwater.

2. It is not effected by the presence or absence of the light as it works on sound waves propagation. Sound waves are also not effected by presence of infrared or higher energy radiations.

3. Sonar can operate at lower frequencies. Lower frequency generates high wavelength acoustic waves which can penetrate through liquid waste present in HLW.

4. Sonar transducer can generate different beam patter which determines the spatial angle and the area covered. This pattern is determined by factors such as the 
frequency of operation and the size, shape and acoustic phase characteristics of the vibrating surface.

5. Temperature, salinity, and pressure are the main factors affecting the performance of sonar. Since pressure will remains constant inside HLW tanks, only salinity and temperature are to be taken into account. These factors cause the sound speed to change. Calibrating for the actual sound speed and inputting it into the sonar software can correct this.

\subsection{Research objective}

The objective of the research is to design and conduct a series of experiments to demonstrate the efficacy of a monitoring system designed by FIU for mapping the settled solid-layer inside HLW tanks. The FIU monitor design consists of a profiling sonar coupled to a mechanical platform to allow its insertion and removal from HLW tanks.

\subsection{Related research}

The term SONAR is an acronym for Sound Navigation and Ranging. One of the earliest references to this concept can be found in a 1490 notebook by an archetypal engineer, Leonardo da Vinci, that stated "If you cause your ship to stop, and place the head of a long tube in the water and place the outer extremity to your ear, you will hear ships at a greater distance from you.’[1].

The importance of being able to visualize submarine and other objects, such as icebergs, was illustrated by the sinking of the Titanic on April 15, 1912 and later by the German U-boat menace to the French shipping fleet in World War I. Sailing at high speed, about 1600 miles northeast of New York City, the Titanic, the world's largest ship, 
on its maiden voyage struck an unseen iceberg and sank with 1517 passengers and crew losing their lives [2]. This shipwreck resulted in a number of patents being issued for iceberg detection using sonar.

In 1912, Sir Hiram S. Maxim, an American- born engineer and inventor, proposed that ships could be protected from collision with icebergs and other ships by generating sound pulses under water and detecting their echoes. Shortly afterwards, two inventors L. F. Richardson in 1912 submitted a British patent and Canada's Reginald A. Fessenden filed a U.S. patent in 1913 for detection of underwater sound. A field trial in April 1914 by R. A. Fessenden resulted in iceberg detection at a range of two miles [3].

Another motivating factor for the development of more sophisticated underwater detection equipment originated from World War I, because of the enormous destructive power of German submarines. An engineer, M. C. Chilowski, developed an ultrasonic device for the French Navy, but its acoustic frequency was too weak to be practical. Paul Langevin (French physicist), heading a joint U.S., British, and French venture, worked on increasing the acoustic power in water and obtained a high ultrasonic intensity by means of piezoelectric transducers [4].

The first active sonar was used by the British Navy in 1918. During this period the typical range of active sonar was 450 meters, while that of passive sonars was 19 kilometers. However, the active sonar operated at higher frequencies $(15-25 \mathrm{kHz})$ and therefore provided a potential angular resolution of approximately one order of magnitude better than the passive sonar [5].

By the start of World War II, every naval vessel engaged in anti-submarine work was equipped with sonar. During this period, a clear understanding of absorption of 
sound in water and accurate values of absorption coefficients were determined and described by Klien [6].

The years since World War II have seen remarkable advances in the exploitation of underwater acoustic for both military and non-military purposes. On the military side, active sonars have grown larger and more powerful and operate at frequencies several orders of magnitude lower than in World War II. As a result, active sonar ranges are greater today than they were during the years of World Wars II and I. Similarly, passive sonars operate at lower frequencies in order to take advantage of the tonal or line components in the low-frequency noise spectrum. By the 1960's, the typical passive sonar range increased to 160 kilometers and the active sonar range about 8 kilometers. However, ships become significantly quieter (less noisy), and the passive sonar detection range available now is probably only a fraction of the value available in the early 1970's for specially designed quiet ships.

A new development of the post war period, which is still taking place today, is the expansion of the application of underwater sound to non-military purposes. Sonars originally employed for depth measurements are now being used for a variety of purposes, such as, for bathymetric mapping of rivers, inspection of bridge and pier supports, dam inspection, pipeline survey, underwater construction monitoring, and in sea for monitoring harbors and shipping channels, to discover the aquatic life in the seabed and in oil reserve exploration.

Recently, the use of multi-beam profiling sonars for ocean-, river- or lake-bottom profiling has been studied. The high resolution mapping of lakes- or ocean-bottom 
reveals features related to hydrothermal, tectonic, volcanic, and sedimentary processes [7].

U.S. Geological Survey has successfully imaged inside Yellowstone lake in Yellowstone national parks by use of multi-beam sonar. Imaged and identified features include over 150 hydrothermal vent sites, several large ( $>500$ meter diameter) and many small hydrothermal explosion craters ( $\sim 1$ to $\sim 200$ meters in diameter), elongated fissures cutting post glacial $(<12 \mathrm{Ka})$ sediments, and submersed former shorelines, all with in the southeast margin of the 0.640-Ma Yellowstone caldera [8]. Also, the importance of multi-beam high resolution profiling sonar to study the fisheries habitat is currently being reviewed by Thales Geosolutions Inc, San Diego, CA, USA. Multi-beam profiling sonars are usually deployed in lakes or rivers varying between 6 to 6000 meters with frequency varying from 12-200 KHz. Images obtained are analyzed to optimize frequency for a particular depth. In addition, the analysis of controlled high-resolution side scan sonar and multi-beam backscattered comparison of 10 square feet section of riverbed is done [9]. One of the main problems caused in the shallow water is due to false detection of objects. Studies have been done to find an optimal solution for this problem, such as, removing clutter (non-target) as possible while maintaining an acceptable detection performance [10]. Research is also carried to increase the effectiveness of profiling sonar in detecting underwater moving objects. Noise present in the water causes signal deviation and reverberation which creates problems in detecting the moving object in water. This phenomenon also causes change in sound velocity and scattering in the water [11]. Multi-beam profiling sonar is now also being used to locate the buried ships or objects in sea water. Researchers are able to locate the shipwreck of an $8^{\text {th }}$ century B.C. at 
the coast of Israel. Profiling sonars operating at a frequency of $150 \mathrm{KHz}$ were capable of accurate measurements of ( $>5$ meter) and reconstruct the structure of the ship [12, 13].

Various models of multi-beam profiling sonars have been developed to improve their performance to get a better system response. Target dimensions, scatter separation, net depth, directivity, pulse length, pulse repetition rate, and wavelength are some of the parameters being studied to improve its performance [14].

Profiling sonars are primarily used for 1) navigational purposes in underwater vehicles (as high resolution and precision are required), 2) in remote operated vehicles (ROV), and 3) in leakage detection in pipe lines [15, 16, 17, and 18]. Most of the sonar research has been related to sea or rivers with unlimited boundaries with varying pressure, temperature, and depth. This research described here deals with use of singlebeam profiling sonar in HLW tanks with close boundaries at constant temperature and pressure.

\subsection{Research methodology}

The main objective of this thesis is to demonstrate the viability and capability of the sonar under various conditions present inside HLW tanks. To achieve this objective, conditions and factors (present inside Hanford HLW tanks) affecting the sonar imaging capability were determined. The most important of these factors were:

1. Radioactivity (mainly gamma radiations);

2. Highly saturated caustic solution having $\mathrm{pH}>14$;

3. Micron-size particles of varying density;

4. Multiple layers of floating solids due to similar density as of caustic solution; 
5. Re-suspension of the solids due to pumping or transfer of fluid;

6. Variation in temperature;

7. Placement and location of sonar inside the HLW tanks.

Experiments were designed to simulate the conditions present inside HLW tanks so as to study the behavior of sonar in these conditions Before proceeding to show the sonar is not effect by these factors, tests were conducted to show sonar's ability to accurately determine the objects placed inside metallic and plastic tanks of smaller diameter $(<7.4$ ft. diameter) as that of Hanford 70-feet HLW tanks.

1. To obtain sonar image of the various objects placed inside the tank. Images of stainless steel drum, a metal object, and a gradient (formed by kaolin clay having $1 \mu$ diameter) at the bottom of the drum were taken. These experiments was conducted in a 7.4-feet and 3.5-feet diameter tanks.

2. To measure interface height and distances between reference points inside tanks using sonar imaging system. Measurements were taken between the sonar head, tank wall, and an interface created by settled kaolin clay. This experiment was conducted to determine the accuracy of the sonar in detecting solid-liquid interface in small tanks.

After proving that sonar works fine in a metallic tank, more experiments were designed to simulate the conditions present inside the Hanford HLW.

1. To obtain accurate sonar measurements under various levels of suspension of solid particles during agitation. Measurements were taken between the sonar head, tank wall, metal pipes, and the settled kaolin clay interface. Solid particles were 
suspended with the help of submersible pump which agitated the water at two different speeds.

2. To image solids having the same density as of water. The purpose of this experiment was to determine the sonar's ability to accurately detect these light solids (having density $1.04 \mathrm{~g} / \mathrm{cm}^{3}$ ). Measurements were taken between the sonar head, tank wall, and settled plastic beads interface. Plastic beads are easily suspended as the density is $4 \%$ more than that of the water.

3. Analysis of sonar-measured, solid layer heights, and distances between reference points when the sonar is located close to a wall or close to the settled layer to be imaged. Multiple measurements were taken when the sonar head was located $<2$ feet from a wall or floor of tank. The results of this experiment were analyzed to find an optimal location for sonar deployment in Hanford HLW tank.

4. To determine the material degradation of the sonar head and cables after exposure to a highly caustic solution $(\mathrm{pH}>14)$. Sonar was placed in a caustic solution (similar to the solution present in Hanford HLW tanks) for specific amount of time at elevated temperature.

5. Analysis of sonar performance in caustic solution. Measurements were taken of two stainless steel objects placed in caustic solution with varying density (1.1 $\mathrm{g} / \mathrm{cm}^{3}, 1.2 \mathrm{~g} / \mathrm{cm}^{3}, 1.3 \mathrm{~g} / \mathrm{cm}^{3}$, and $\left.1.4 \mathrm{~g} / \mathrm{cm}^{3}\right)$ and at different temperatures $\left(25^{\circ} \mathrm{C}\right.$, $30^{\circ} \mathrm{C}$, and $35^{\circ} \mathrm{C}$ ). Results were analyzed to calibrate the sonar measurements by calculating correct sound speed specific density and temperature.

Also modeling and simulation of working of profiling sonar is done with the help of general sonar equations. Results obtained from this simulations were analyzed to design 
these experiments. Also software was generated based on the results of Experiment 5 to correct the sound speed in a medium.

\subsection{Overview of thesis chapters}

Chapter 1 covers the research problem addressed, the objective of this thesis, and background on relevant research by others in the area of sonar. Chapter 2 provides an overview of sonar equipment and theory that includes hardware design, and sonar equations. Also simulation and modeling of working of profiling sonar is done. Chapter 3 presents the experimental set-up, experimental procedure, and the experimental design for experiments and their results. A summary briefly reviewing the analyses of the results are also described in this chapter. The images obtained experimentally are also corrected by using the correct sound speed. Finally chapter 4 contains conclusions from the experimental research with emphasis on relevance of the results overall and potential future research suggested by this research.

\subsection{Summary of thesis research}

The objective of this research was met as my experiments showed that we could map settled solids with accurate measurements. Measurements were accurate even with $30 \%$ solids (by weight) entrained in water. Modeling done with the help of FEMLAB and Matlab showed how a acoustic wave propagates in a medium and how a sonar image is generated. Also program written in Visual Basic 6 solved the problem of getting wrong measurements for different density liquids. The software written was combined with the original sonar software to correct the measurements.

Unique research accomplished here includes: 
1. Design of a small-scale experiment using profiling sonar to map the settled solids layer in a vessel;

2. Calculated the transmission loss of sound wave pulse for Imagenex $881 \mathrm{~A}$ profiling sonar for different ranges;

3. Optimize the performance of a profiling sonar in caustic solutions without taking density and concentration of solution into account;

4. Mapping of interface using a profiling sonar by rotating the sonar around its head at small angles;

5. The program written in V.B.6 is a unique research as it corrects the commercial available sonar software to generate exact measurements in the images when there is change in density or temperature of the medium.

Finally the simulation done using FEMLAB and Matlab was not unique research but did explain the phenomenon of generation of multiple images of the interface when range is large. The results obtained from the simulation helped in designing the experiments. 


\section{Chapter 2}

\section{SONAR EQUATIONS AND THEORY}

\subsection{General Description of sonar systems}

Sonar refers to the application of sound for the detection and the location of underwater objects. Since electromagnetic radiations, such as visible light or radar, do not penetrate through water significantly, sonar is the most successful method for underwater detection. The simplest sonar devices send out a sound pulse from a transducer and then precisely measure the time it takes for the sound pulses to be reflected back to the transducer. The distance to an object can be calculated using this time difference and the speed of sound in the medium. There are two types of the sonar: passive and active sonar. 1. Passive sonar:

Passive sonar is a listening device; sound waves produced by another source are received by the sonar's receiver and changed into electrical signals for display on a monitor.

2. Active sonar:

Active sonar is able to both send and receive signals. Active sonar uses a transducer, which converts electrical signal to sound waves. These sound waves are reflected back from the target and detected by the sonar's receiver as an echo. The receiver passes sound waves to the transducer which converts the sound back to electrical signals. Since the speed of the sound in water is known, range and the bearing of the target can be determined. This method is also called echo-ranging. 


\subsection{Description of terms used in sonar equations}

The equations are founded on a basic equality between the desired and undesired portions of the received signal at the instant when some function of the sonar set is just performed. These functions may be detection of an underwater target or other acoustic activities. These functions involve the reception of the acoustic energy occurring in a natural acoustic background. Of the total acoustic field at the receiver, a portion is desired and is called the signal. The remainder of the acoustic field is undesired and is called the background. In sonar the background is either noise, i.e., the essentially steady-state portion not due to one's own echo ranging, or reverberation, but the slowly delayed portion of the background representing the return of one's own acoustic output by scatters in the medium. For better performance of the sonar the overall response of the system to the signal is increased and its response to the background is decreased. A signal can be detected when its level equals the level of the background.

$$
\text { Signal level }=\text { background masking level }
$$

The equality mentioned exists only at one instant in time, as the target approaches or recedes from the sonar receiver. At short ranges, its signal will exceed the background masking level but at long ranges, the reverse will occur.

The basic equations can be expanded in term of various parameters. These parameters are determined by the equipment, the medium, and the target. These parameters are level in units of decibel, as follows:

1. The Equipment

Projector Source Level: SL

Self-Noise Level: NL 


\section{Receiving Directivity Index: DI}

Detection Threshold: DT

2. The Medium

Transmission Loss: TL

Reverberation Level: RL

Ambient-Noise Level: NL

\section{The Target}

\section{Target Strength: TS}

Target Source Level: SL

The two pairs of the parameters e.g., Projector Source Level (SL) and Target Source Level (SL) are given the same symbol because they are essentially identical. It should be noted in passing such set of parameters is not unique. For example, sound velocity could be adopted as a parameter, and TS could be replaced by the parameter: "backscattering cross section" expressed in decibels. The chosen parameters are arbitrary and those employed here are the ones conventionally used in underwater sound. There are no conventional symbols for these parameters.

1. Source Level $(S L)$ : Source level is defined differently for active and passive sonar equations. For the active sonar equations, it is the sound pressure level of the actively transmitting sonar, measured (or referenced to) one yard from the transducer. For the passive sonar equation, the source level is the measure of the noise generated by the object at specific frequencies and is also referenced to one yard from the sound source.

2. Directivity Index (DI): Directivity index indicates the amount by which a sonar, uses its directional beam forming capability by discriminating omni directional noise from a 
directional signal. The directivity index is a function of the sonar's design and the received frequency only. Directivity index is the measure of the amount by which a given sonar system can filter out background noise by using its directional beam forming capability. The value for the directivity index for a specific system will always be a positive value. Normally the value of DI is taken as Zero (0) when working with the equations.

3. Detection Threshold (DT): Detection threshold is signal-to-noise ratio required for a $50 \%$ probability of detection (POD) of the object. The value for the Detection threshold for a specific operator will always be a negative number. DT is the means to account the ability of sonar to detect object noise which in most cases is more than the surrounding noise.

4. Transmission Loss (TL): Transmission loss is defined by the decrease in acoustic intensity of an acoustic pressure wave propagating outwards from a source. As the acoustic wave propagates outwards from the source, the intensity of the signal is reduced with increasing range due to spreading and attenuation.

5. Self Noise level (NL), Reverberation Level (RL), and Ambient-Noise Level (NL): The noises which are present in a medium and which a sonar has to overcome to detect an object are represented by the noise level (NL) term. NL is actually a combination of several terms. In the passive sonar equation, NL is the summation of two noise sources: self noise ( $\mathrm{SN}$ ) and ambient noise (AN). In the active sonar equations, NL is either the summation of $\mathrm{SN}+\mathrm{AN}$, identical to the passive sonar equations, or it is the amount of measured reverberation (RL). It is this difference in NL terms that gives rise tothe two active sonar equations; one with self noise/ambient noise and one with reverberation as 
the noise level term. Normally these noise levels and measurements are omni directional (all directions) in nature.

6. Target Strength (TS): Target strength applies to the active sonar equation only. This term is added to the source level term and accounts for the sound energy that reflects off an object. Specifically, TS is the ratio of incident sound energy to reflected sound energy. It is dependent upon the cross-sectional area from which the sound wave-front reflects (target aspect) and the object material or geometry.

Table 1 summaries the parameters discussed above.

Table 1: Sonar Parameters, their Definitions, and Reference Locations

\begin{tabular}{|l|l|l|l|}
\hline Parameters & Symbols & $\begin{array}{l}\text { Reference } \\
\text { Locations }\end{array}$ & Definitions \\
\hline Source level & SL & $\begin{array}{l}\text { 1 yd from the } \\
\text { source on its } \\
\text { acoustic axis }\end{array}$ & $10 * \log \frac{\text { intensity of source at lyd }}{\text { reference intensity }}$ \\
\hline $\begin{array}{l}\text { Transmission } \\
\text { loss }\end{array}$ & TL & $\begin{array}{l}\text { 1 yd from } \\
\text { source and at } \\
\text { target or } \\
\text { receiver }\end{array}$ & $10 * \log \frac{\text { signal intensity at lyd }}{\text { siganl intensity at target or reciever }}$ \\
\hline Target loss & TS & $\begin{array}{l}\text { 1 yd from } \\
\text { acoustic } \\
\text { center of } \\
\text { target }\end{array}$ & $10 * \log \frac{\text { echo intensity at lyd from terminal }}{\text { incident intensity }}$ \\
\hline Noise level & NL & $\begin{array}{l}\text { At } \\
\text { hydrophone } \\
\text { location }\end{array}$ & $10 * \log \frac{\text { noise intensity }}{\text { reference intensity }}$ \\
\hline $\begin{array}{l}\text { Receiving } \\
\text { directivity } \\
\text { index }\end{array}$ & DI & $\begin{array}{l}\text { At } \\
\text { hydrophone } \\
\text { terminals }\end{array}$ & $\begin{array}{l}10 * \log \\
\text { noise power generated by an equivalent } \\
\text { nondirectional hydrophone }\end{array}$ \\
\hline $\begin{array}{l}\text { Reverberatio } \\
\text { n level }\end{array}$ & RL & $\begin{array}{l}\text { At } \\
\text { hydrophone } \\
\text { terminals }\end{array}$ & $\begin{array}{l}10 * \log \\
\text { reverberaton powerat hydrophoneteminal }\end{array}$ \\
\hline $\begin{array}{l}\text { Detection } \\
\text { threshold }\end{array}$ & DT & $\begin{array}{l}\text { At } \\
\text { hydrophone } \\
\text { terminals }\end{array}$ & $\begin{array}{l}10 * \log \\
\text { signal power to just perform a certain function }\end{array}$ \\
\hline
\end{tabular}




\subsection{Sonar equations}

Consider a sound source acting also as a receiver (a transducer) that produces a source level of SL decibels at a unit distance (1 yd) on its axis. When the radiated sound reaches the target (if the axis of the sound points towards the target), its transmission will be reduced by the transmission loss, and becomes SL-TL. On scattering or reflection by the target of target strength $\mathrm{TS}$, the reflected or the backscattered level will be SL-TL+TS at a distance of $1 \mathrm{yd}$ from the acoustic center of the target in the direction back towards the source. In traveling back toward the source, this level is again attenuated by the transmission loss and becomes SL-2TL+TS. This is the echo level at the transducer. Assuming that the background noise is isotropic noise rather than reverberation, the background level becomes NL. This level is reduced by the directivity index of the transducer acting as receiver or hydrophone so that at the terminal of the transducer the relative noise power is NL-DI. Since the axis of the transducer is pointing in the direction from which the echo is coming, the relative echo power is unaffected by the transducer directivity. At the transducer terminals, the echo-to-noise ratio is:

$$
\text { SL-2TL+TS-(NL-DI) }
$$

When the input signal-to-noise ratio is above a certain detection threshold fulfilling certain probability criteria, a decision is made that target is present. When the input signal-to-noise ratio is less than the detection threshold, then target is absent. When the

target is just detected, the signal-to-noise ratio equals the detection threshold, and equation becomes:

$$
\mathrm{SL}-2 \mathrm{TL}+\mathrm{TS}-(\mathrm{NL}-\mathrm{DI})=\mathrm{DT}
$$


Equation 2.3 is an active sonar equation in terms of the detection threshold, also called recognition differential. In terms of the basic equality described it could be consider that only that part of noise power lying above the detection threshold level mask the echo, and the equation becomes:

$$
\mathrm{SL}-2 \mathrm{TL}+\mathrm{TS}=\mathrm{NL}-\mathrm{DI}+\mathrm{DT}
$$

This is a more convenient arrangement of the parameters, since the echo level occurs on the left-hand side, and the noise-masking background level occurs on the right. This is the active sonar equation for the mono-static case in which the source and the receiving hydrophones are coincident and in which the acoustic return of the target is back towards the source. In some sonar, a separate source and receiver are employed and the arrangement is said to be bi-static; in this case the two transmission losses to and from the target are not the same. Also in some sonars it not possible to distinguish between DI and DT, and it becomes equal to DI-DT as the increase in signal-to-background ratio produced by the entire receiving system of transducer, electronics, display, and observer.

When the background noise is due to reverberation, the parameter DI, defined in terms of an isotropic background, is inappropriate. For a reverberation background the term NL-DI is replaced by an equivalent plane wave reverberation level RL observed at the hydrophone terminals. The active sonar equation then becomes:

$$
\mathrm{SL}-2 \mathrm{TL}+\mathrm{TS}=\mathrm{RL}+\mathrm{DT}
$$

There are separate names for different combinations of the terms in the sonar equations. Table 2 contains the list of names for the different combination of terms. 
Table 2: Terminology of Various Combinations of the Sonar Parameters

\begin{tabular}{|c|c|c|}
\hline Terms & Definitions & Remarks \\
\hline Echo level & $\mathrm{SL}-2 \mathrm{TL}+\mathrm{TS}$ & $\begin{array}{l}\text { The intensity of the echo as measured in the } \\
\text { water at the hydrophones. }\end{array}$ \\
\hline $\begin{array}{l}\text { Noise masking } \\
\text { level }\end{array}$ & $\mathrm{NL}-\mathrm{DI}+\mathrm{DT}$ & \multirow{2}{*}{$\begin{array}{l}\text { Another name for these two combinations is } \\
\text { minimum detectable echo level. }\end{array}$} \\
\hline $\begin{array}{l}\text { Reverberation } \\
\text { masking level }\end{array}$ & $\mathrm{RL}+\mathrm{DT}$ & \\
\hline Echo excess & $\begin{array}{l}\mathrm{SL}-2 \mathrm{TL}+\mathrm{TS}- \\
(\mathrm{NS}-\mathrm{DI}+\mathrm{DT})\end{array}$ & $\begin{array}{l}\text { Detection occurs when echo excess is zero } \\
\text { under the probability conditions implied in } \\
\text { the term DT. }\end{array}$ \\
\hline Performance figure & $\mathrm{SL}-(\mathrm{NL}-\mathrm{DI})$ & $\begin{array}{l}\text { Difference between the source level and the } \\
\text { noise level measured at the hydrophone } \\
\text { terminals. }\end{array}$ \\
\hline Figure of merit & $\mathrm{SL}-(\mathrm{NL}-\mathrm{DI}+\mathrm{DT})$ & $\begin{array}{l}\text { The maximum allowable two-way loss for } \\
\text { TS = } 0 \text { decibels in active sonars. }\end{array}$ \\
\hline
\end{tabular}

Of these, the Figure of merit (FOM) is the most useful, because it combines together the various equipment and the target parameters so as to yield a quantity significant for the performance of the sonar. Since it equals the transmission loss at the instant when the sonar equation is satisfied, the FOM gives an intermediate indication of the range at which a sonar can detect its target, or more generally, perform its function. However, when the background is reverberation instead of the noise, the figure of merit is not constant, but varies with range and so fails to be a useful indicator of the sonar performance. While the Figure of Merit is the calculated sum of the sonar equation terms, and is defined by the maximum loss a signal can suffer and still be detected (recognized) $50 \%$ of the time. The FOM definition forms the basis for sonar range prediction; where the FOM value equals the propagation loss, there is a $50 \%$ probability of detection.

In the case of active sonar range prediction, Active Figure of Merit (AFOM) is used. The TL term is doubled when calculating AFOM due to two-way sound travel. In many cases, it is needed to determine counter detection ranges; that is, the predicted 
ranges within which own sonar will be detected by another sonar or object. In these cases, the Figure of Demerit (FDM) is computed.

\subsubsection{Transient form of the sonar equations}

The equations discussed so far have been written in terms of intensity, or the average acoustic power per unit area of the sound emitted by the source or received from the target. The word average implies a time interval over which the average is to be taken. The time interval causes uncertain results for short transient sources or generally, whenever severe distortion is introduced by propagation in the medium or by scattering from the target.

A more general approach is to write the equations in terms of energy flux density defined as the acoustic wave using a time-varying pressure $\mathrm{p}(\mathrm{t})$; then the energy flux of the wave is:

$$
E=\frac{1}{\rho c} \int_{0}^{\infty} p^{2}(t) d t
$$

The units of pressure are dynes per square centimeter and the acoustic impedance of the medium is ergs (for water, $\rho c \approx 1.5 \times 10^{5}$ ), then $\mathrm{E}$ is expressed in ergs per square centimeter. The intensity is the mean square pressure of the wave divided by $\rho c$ and averaged over an intensity of time $\mathrm{T}$, or

$$
I=\frac{1}{T} \int_{0}^{T} \frac{p^{2}(t)}{\rho c} d t
$$

So that over the time interval $\mathrm{T}$,

$$
I=\frac{E}{T}
$$


The quantity $\mathrm{T}$ is the time interval over which the flux density of an acoustic wave is to be averaged to form the intensity. For long pulse active sonars, this time interval is the duration of the emitted pulse and is very nearly equal to the duration of the echo. For short transient sonars, however, the interval $\mathrm{T}$ is often ambiguous, and the duration of the echo is vastly different from the duration of the transient emitted from the source. Under these conditions, it can be shown [19] that the intensity from the sonar equations can be used, provided that the source level is defined as:

$$
\mathrm{SL}=10 \log (\mathrm{E})-10 \log \left(\mathrm{r}_{\mathrm{e}}\right)
$$

Where $\mathrm{E}$ is the energy flux density of the source at $1 \mathrm{yd}$ and is measured in units of the energy flux density of a $1 \mu \mathrm{Pa}$ plane wave taken over an interval of a 1 second and $r_{e}$ is the duration of the echo in seconds for an active sonar depth. For pulsed sonars emitting a flat topped pulse of constant source level SL over a time interval $r_{o}$ then,

$$
10 \log (\mathrm{E})=\mathrm{SL}^{\prime}+10 \log \left(\mathrm{r}_{\mathrm{o}}\right)
$$

Since the energy density of a pulse is the product of the average intensity times its duration, by combining the best two equations, the effective source level SL for use in the sonar equations is therefore:

$$
\mathrm{SL}=\mathrm{SL}^{\prime}+\log \frac{\mathrm{r}_{\mathrm{o}}}{\mathrm{r}_{\mathrm{e}}}
$$

Here $r_{o}$ is the duration of the emitted pulse of the source level SL', and $r_{e}$ is the echo duration. For long-pulsed sonar, $\mathrm{r}_{\mathrm{o}}=\mathrm{r}_{\mathrm{e}}$ and $\mathrm{SL}=\mathrm{SL}$ '. For short- pulsed sonars, $\mathrm{r}_{\mathrm{e}}>\mathrm{r}_{\mathrm{o}}$ and the effective source level SL is less than $\mathrm{SL}^{\prime}$ by the amount $10 \log \left(\mathrm{r}_{\mathrm{o}} / \mathrm{r}_{\mathrm{e}}\right)$. A short pulse of duration $\mathrm{r}_{\mathrm{o}}$ and source level $\mathrm{SL}^{\prime}$ is replaced in a sonar calculation by an effective or 
equivalent pulse of longer duration $r_{e}$ and lower source level SL. The two source levels are related so as to keep the energy flux-density source level the same, namely:

$$
\mathrm{SL}+10 \log r_{\mathrm{e}}=\mathrm{SL}^{\prime}+10 \log \mathrm{r}_{\mathrm{o}}
$$

or

$$
\mathrm{SL}=\mathrm{SL}^{\prime}+\log \frac{\mathrm{r}_{\mathrm{o}}}{\mathrm{r}_{\mathrm{e}}}
$$

In effect, the pulse emitted by the sonar is stretched out in time and thereby reduced in level by the multi path propagation and by target reflection.

The echo duration can be conceived as consisting of three components: $r_{o}$, the duration of the emitted pulse measured near the source; $r_{m}$, the additional duration imposed by the two way propagation in the medium; and $\mathrm{r}_{\mathrm{t}}$, the additional duration imposed by the extension in range of target. So the echo duration is the sum of the three components:

$$
r_{e}=r_{o}+r_{t}+r_{m}
$$

\subsubsection{Applications of the sonar equations}

Sonar equations serve two important practical functions:

\section{Prediction of the performance of sonar equipment of known design:}

In this application the design characteristics of the sonar are known or assumed, and what is desired is an estimate of the performance in terms of detection probability or search rate. This is done by a prediction of range through the parameter transmission loss. The equations are solved for transmission loss, which is then converted to range through some assumption concerning the propagation characteristics of the medium. 


\section{Sonar design:}

This application is used where a pre-established range is required for the operation of the equipment being designed. In this case the equations are solved for the particular parameter of interest.

\subsubsection{Limitations of the sonar equations}

Sonar equations have the following limitations.

1. The sonar equations written in terms of intensities are not always complete for some types of sonars. Short-pulse sonar requires the addition of another term, the echo duration, to account for the time stretching producer by multi path propagation. Another such addition is a correlation loss in correlation sonars to account for the décor-relation of the signal that may occur due to bottom reflection or scattering in bottom-bounce sonars. 2. A limitation of another kind is produced by the nature of the medium in which sonar operates. If the medium is moving and contains in-homogeneities such as irregular boundaries, then many sonar parameters fluctuate irregularly with time, while others change because of the unknown changes in the equipment and the platform to which it is mounted. Because of these fluctuations, a solution to the sonar equations is no more than a best guess time average of what is to be expected in a basically stochastic problem.

3. Precise calculations, to tenths of a decibel, are futile: a predicted sonar range is an average quantity about which the observed values of range are likely to congregate. Underwater sound and its fluctuations, improve the accuracy of the predictions of the sonar equations can be expected to increase. 


\subsection{Sonar equipment used}

The profiling sonar used for this research was the Imagenex Model 881A. A profiling sonar works by sending a series of narrow angle (pencil beam) acoustic pulses out in the direction perpendicular to the axis of the sonar head cylinder (Figure 1). The azimuthal angle of each successive pulse continues to change as the system scans across the desired sector angle. The shape and spacing of the sonar pulses in a single scan varies based upon the selection of the transducer frequency, scan speed, and total sector angle. At the highest frequency $(1 \mathrm{MHz})$ and the slowest scan speed $(0.3$ degree between successive pulses) the system has its highest resolution.

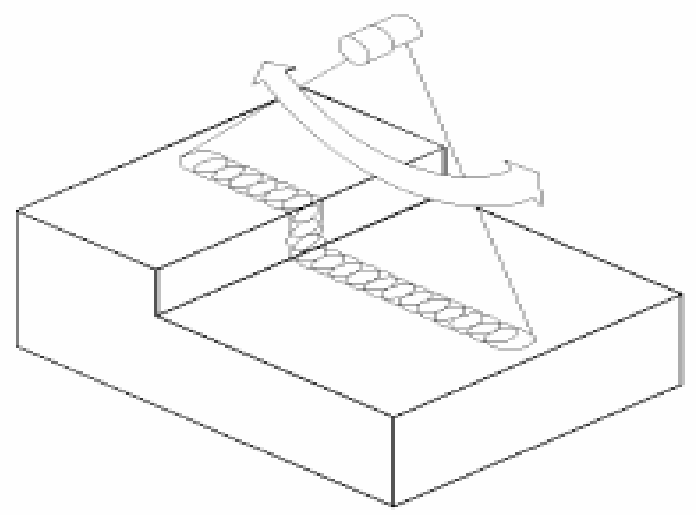

Figure 1: Scanning of profiling sonar over a $120^{\circ}$ sector.

\subsubsection{Hardware description}

Figure 2 shows the device with a quarter placed on the sonar for size comparison. The frequency of the system is tunable to any of the three frequencies: $600 \mathrm{kHz}, 675 \mathrm{kHz}$, and $1 \mathrm{MHz}$ by using software. The beam width is $2.4^{\circ}$ at $600 \mathrm{kHz}, 2.1^{\circ}$ at $675 \mathrm{kHz}$, and $1.4^{\circ}$ at $1 \mathrm{MHz}$. The transducer is housed in a protective fluid filled housing. Three different modes Polar Mode, Sector Mode, or Single Side Scan mode can be selected 
using software configuration. The power supply to the device is 20-36 Volts at less than 5 W. The hardware interface consists of RS-485 at $115.2 \mathrm{~K}$ baud. The cable length is $1000 \mathrm{~m}$ with twisted shielded pair. The material of construction is Titanium chosen to give the required corrosion resistance. Overall dimension of the cylindrical transducer is $3 \frac{1}{4}$ in. diameter $\times 9 \frac{3}{4}$ in. length. The weight of the unit is $2.2 \mathrm{lbs}$ in air. Detailed specifications for the unit can be found in Appendix A.

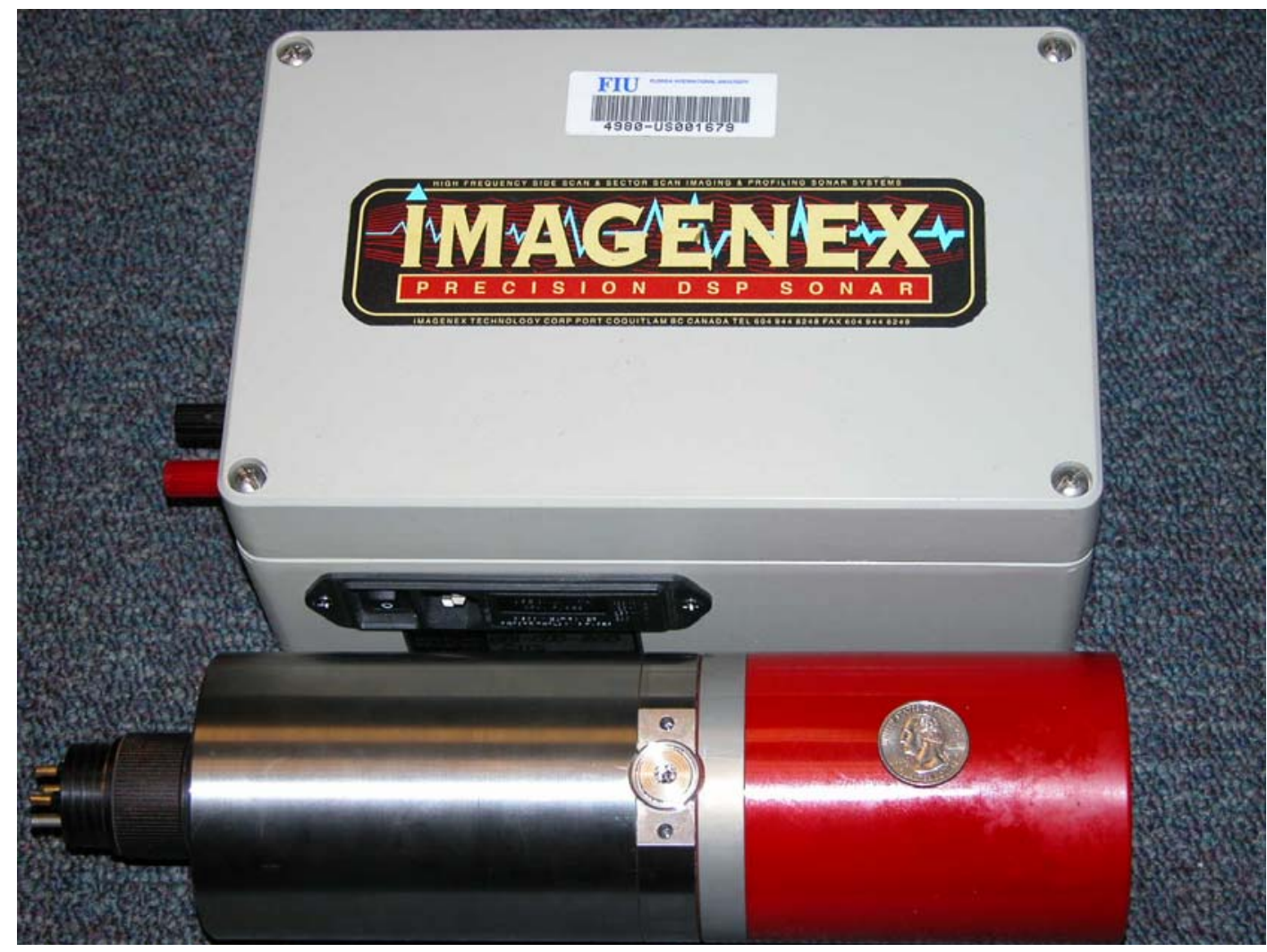

Figure 2: Profiling sonar and data acquisition box.

\subsubsection{Software description}

The software used for data acquisition and display is custom software developed by Imagenex Inc. WIN881A is a Windows 95/98/Me/NT/2000/XP program that controls, displays and records data from the multi-frequency Model 881A Profiling Sonar Head. 
The program uses a 2-Wire RS-485 COM port $(115200, \mathrm{~N}, 8,1)$ to communicate with the head and an RS-232 COM port $(4800, \mathrm{~N}, 8,1)$ for receiving GPS Lat/Lng coordinates. The head can be operated at different ranges, gains, speeds, frequencies, etc. The Windows display mode must be at least $800 \times 600$ pixels with small fonts selected. The following is a screen shot from the software. Detailed expiation of software setting is shown in Appendix B.

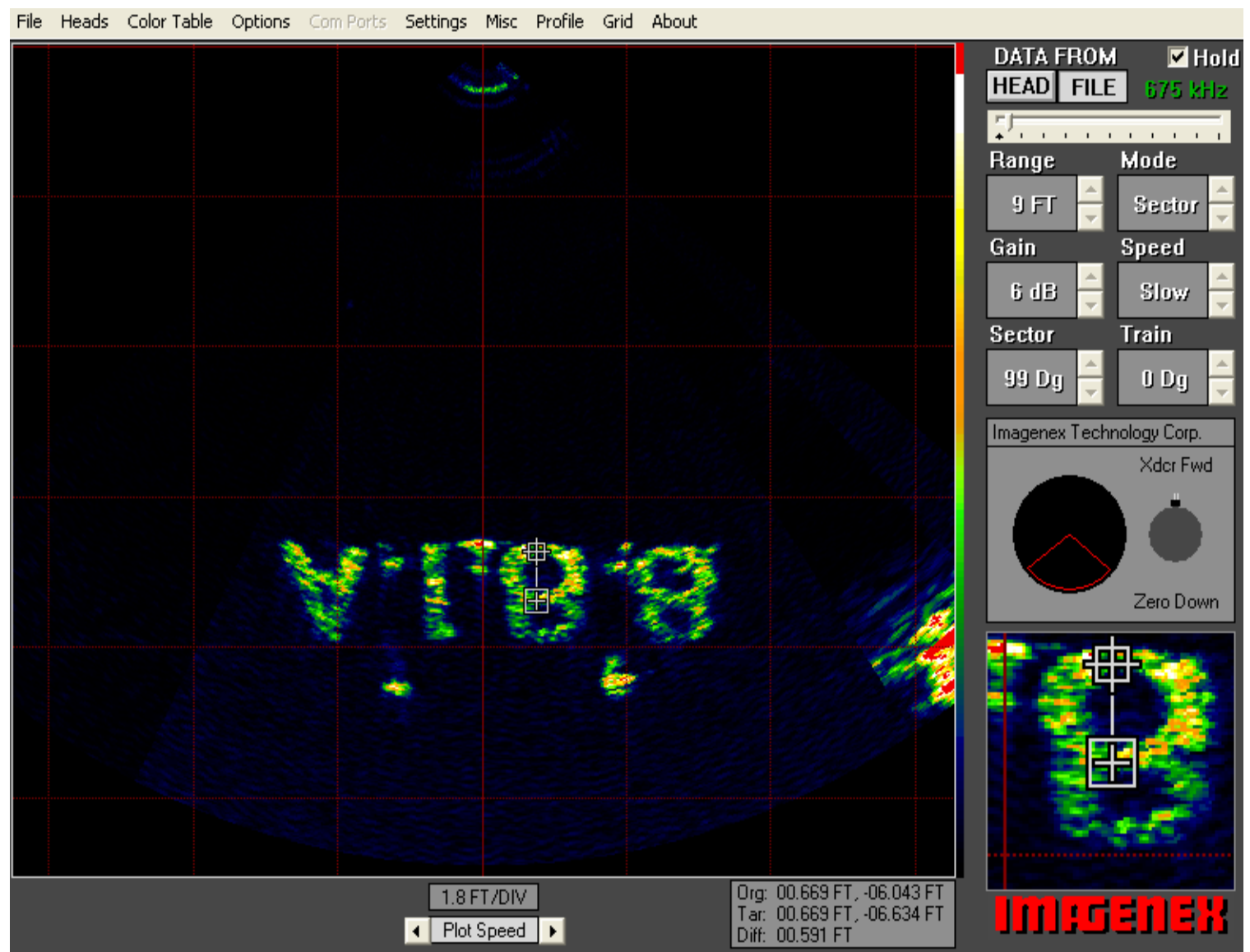

Figure 3: Image displaying the output of sonar with two-cursor measurement and pixel zoom command.

\subsection{Calculations of transmission loss for Imagenex 881A profiling sonar}

The sonar parameter transmission loss describes the weakening of sound between a point $1 \mathrm{yd}$ from the source and a point at a distance in the medium. More specifically, if 
$I_{o}$ is the intensity at the reference point located $1 \mathrm{yd}$ from the acoustic center of the source (10 $\log \mathrm{I}_{\mathrm{o}}$ is the source level of the source) and $\mathrm{I}_{1}$ is the intensity at a distance point, then transmission loss between the source and the distance point is:

$$
\mathrm{TL}=10 \log \frac{\mathrm{I}_{\mathrm{o}}}{\mathrm{I}_{1}} \mathrm{~dB}
$$

Transmission loss depends on mainly two factors: spreading and attenuation

\section{Spreading}

Spreading is further categorized into spherical and cylindrical spreading.

a) Spherical (Free-field spreading): Spherical spreading occurs when the sound spreads uniformly over a sphere or hemisphere (Figure 4) that expands with distance.

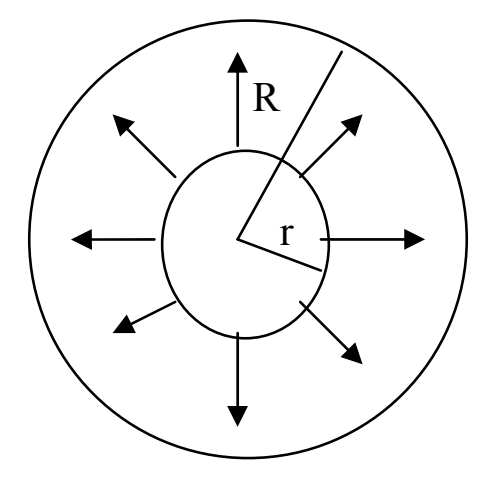

Figure 4: Free field spreading.

The intensity at range $\mathrm{R}$ is given by the power P per unit area. It can be inferred that the circular area over which the power is distributed at a range $\mathrm{R}$ is given by $\pi r^{2}$. Also, the radius of the circular area increases in proportion with the range R. Thus, the intensity is given by:

$$
\mathrm{I}=\frac{\mathrm{p}}{\pi \mathrm{r}^{2}}
$$


and since $\mathrm{r}$ is proportional to $\mathrm{R}$,

$$
\mathrm{I}=\frac{1}{\mathrm{R}^{2}}
$$

Equation 2.17 is the inverse square law which tells that the acoustic intensity is reduced in proportion to the square of the range due to spreading alone.

The formal definition of spreading on the decibel scale is given by Equation 2.20

$$
\begin{aligned}
& \mathrm{TL}=10 \log \left(\frac{\mathrm{P} / 2 \pi \mathrm{1}^{2}}{\mathrm{P} / 2 \pi \mathrm{R}^{2}}\right) \\
& \mathrm{TL}=10 \log \left(\mathrm{R}^{2}\right) \\
& \mathrm{TL}=20 \log (\mathrm{R})
\end{aligned}
$$

b) Cylindrical spreading: The spherical spreading law will apply when sound energy spreads outwards with no refraction or reflection from boundaries. However, in shallow water there are reflections from the surface, and spreading is considerably reduced by refraction and reflection. Under these conditions a cylindrical spreading law of the following form is appropriate:

$$
\mathrm{TL}=10 \log (\mathrm{R})^{2}
$$

However, since sound energy is not perfectly contained by reflection (reflection coefficients less than 1) and refraction, the correct spreading is often somewhere between the predictions given by Equations 2.20 and 2.21. A practical spreading equation which represents an intermediate spreading condition between spherical and cylindrical spreading is given by Urick in his book "Principle of Under Water Sound":

$$
\mathrm{TL}=15 \log (\mathrm{R})^{2}
$$




\section{Attenuation (or absorption)}

Transmission loss due to attenuation is represented in the sonar equations in terms of an attenuation coefficient ' $\alpha$ ' with the units of $\mathrm{dB} / \mathrm{m}$. There are two primary causes of attenuation:
a) Viscous friction
b) Ionic relaxation phenomena

Attenuation due to viscous friction refers to the conversion of sound energy to heat due to internal friction at a molecular scale within the fluid. Viscous friction is the dominant mode of attenuation at frequencies above $1 \mathrm{MHz}$. The attenuation coefficient is strongly frequency dependent with attenuation increasing rapidly with frequency. An approximate expression given by Waite Ashley D.[19] for the attenuation coefficient $\left(\alpha_{1}\right)$ for water due to viscous friction is:

$$
\alpha_{1}=\left(2.1 \times 10^{-10}(\mathrm{~T}-38)^{2}+1.3 \times 10^{-7}\right) \mathrm{f}^{2} \mathrm{~dB} / \mathrm{m}
$$

Here $\mathrm{T}$ is the temperature in centigrade and $\mathrm{f}$ is the frequency in kilo-hertz. This equation is valid for frequencies above $500 \mathrm{kHz}$. At frequencies below about $500 \mathrm{kHz}$ the presence of certain dissolved salts in water increase the attenuation coefficient. The absorption is dominant below $100 \mathrm{kHz}$ due to the ionic relaxation of different salts. The ionic relaxation process involves the disassociation and re-association of different salts ions in water due to the pressure fluctuation resulting from the propagation of the sound wave. A empirical absorption coefficient which accounts for the effect of salts relaxation is given by Urick [20]:

$$
\alpha_{2}=b * f_{0}\left(1+\left(f_{0} / f\right)^{2}\right)^{-1} d B / m
$$

where, $b=2 S \times 10^{-5}$ and $f_{0}=50(T+1), S$ is the salinity of the medium. 
The overall absorption due to viscous friction and salts relaxation is given by the sum of Equations 2.23 and 2.24 shown below:

$$
\alpha=\alpha_{1}+\alpha_{2} .
$$

The combined effect of spreading and absorption are given by:

$$
\mathrm{TL}=20 \log (\mathrm{R})+\alpha \mathrm{R}^{2}
$$

Imagenex $881 \mathrm{~A}$ profiling sonar was tested in tanks filled with tap water. Since the tanks were not bigger than $7 \mathrm{ft}$ in height and $7.4 \mathrm{ft}$ in diameter pressure didn't change. Also the salinity of the tap water is less than $1000 \mathrm{mg} / \mathrm{l}$ that is small and can be neglected. Only factor which effects the attenuation coefficient in these experiments is temperature. So the Equations 2.26 and 2.25 were used. In Equation $2.25 \alpha_{2}$ was always taken equal to zero as salinity is very less and more over the frequencies used are far more than 100 $\mathrm{kHz}$. Only parameter which is not considered in TL modeling is the anomaly caused by water due to the traveling of acoustic pressure wave [21]. Usually it's a number and is denoted by A. It is added to the Equation 2.26 to get the proper value.

$$
\mathrm{TL}=20 \log (\mathrm{R})+\alpha \mathrm{R}^{2}+\mathrm{A}
$$

For sea water the value of $\mathrm{A}$ is considered as 5 at $4^{\circ} \mathrm{C}$. This value keeps on changing with the temperature. For the modeling purpose A is not considered since its value for tap water at particular temperature is not known.

$$
\alpha=\left(2.1 \times 10^{-10}(\mathrm{~T}-38)^{2}+1.3 \times 10^{-7}\right) \mathrm{f}^{2}
$$

Taking $\mathrm{T}=25^{\circ} \mathrm{C}$ and frequency $\mathrm{f}=600 \mathrm{kHz}$

$$
\alpha_{600}=\left(2.1 \times 10^{-10}(25-38)^{2}+1.3 \times 10^{-7}\right) \times(600)^{2}
$$




$$
\alpha_{600}=0.06 \mathrm{~dB} / \mathrm{m}
$$

Similarly attenuation coefficient for $\mathrm{f}=675 \mathrm{kHz}$ and $\mathrm{f}=1000 \mathrm{kHz}$ at $25^{\circ} \mathrm{C}$ are

$$
\alpha_{675}=0.08 \mathrm{~dB} / \mathrm{m} \text { and } \alpha_{1000}=0.17 \mathrm{~dB} / \mathrm{m}
$$

Transmission losses were calculated over a specified range at three frequencies $600 \mathrm{kHz}$, $675 \mathrm{kHz}$, and $1 \mathrm{MHz}$. by using equation 2.26 .

\begin{tabular}{|c|c|c|c|}
\hline \multirow{2}{*}{ Range (ft) } & \multicolumn{3}{|c|}{ Transmission Loss } \\
\hline & $600 \mathrm{kHz}(\mathrm{dB})$ & $675 \mathrm{kHz}(\mathrm{dB})$ & $1 \mathrm{MHz}(\mathrm{dB})$ \\
\hline 3 & 9.722425 & 9.782425 & 10.05243 \\
\hline 6 & 15.92303 & 16.04303 & 16.58303 \\
\hline 9 & 19.62485 & 19.80485 & 20.61485 \\
\hline 12 & 22.30362 & 22.54362 & 23.62362 \\
\hline 15 & 24.42183 & 24.72183 & 26.07183 \\
\hline 30 & 31.34243 & 31.94243 & 34.64243 \\
\hline 60 & 39.16303 & 40.36303 & 45.76303 \\
\hline 90 & 44.48485 & 46.28485 & 54.38485 \\
\hline 120 & 48.78362 & 51.18362 & 61.98362 \\
\hline 150 & 52.52183 & 55.52183 & 69.02183 \\
\hline 180 & 55.90545 & 59.50545 & 75.70545 \\
\hline 240 & 62.00422 & 66.80422 & 88.40422 \\
\hline 300 & 67.54243 & 73.54243 & 100.5424 \\
\hline 450 & 80.06425 & 89.06425 & 129.5643 \\
\hline 600 & 91.56303 & 103.563 & 157.563 \\
\hline
\end{tabular}

Table 3: Range and Transmission Loss for Three Frequencies at $25^{\circ} \mathrm{C}$.

Transmission losses were plotted with their respective ranges in the following Figure 5.

From the graph it can be seen that transmission loss is less at lower frequencies and more 
at higher frequencies. Also transmission loss is range dependent, it increases with the range.

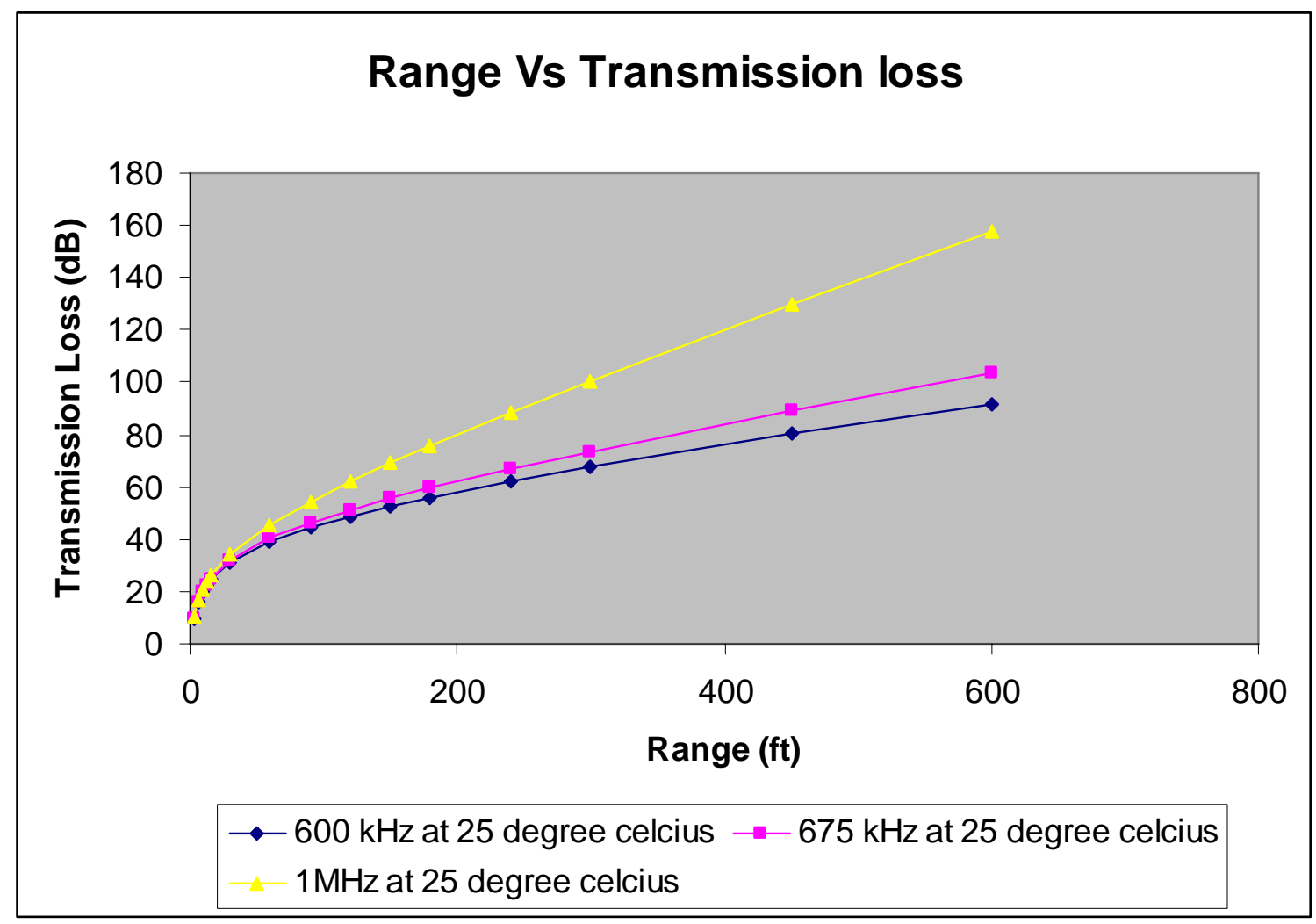

Figure 5: Transmission loss over a specified range at $25^{\circ} \mathrm{C}$ for three frequencies.

\subsection{Sonar simulations}

Sonar systems require realistic acoustic waveforms to test their beam forming, classification, and tracking. Entire system needs to be tested with self-consistent and reproducible data, and exhaustive testing specially with caustic and radioactive environment. An alternative is to synthesize hydrophone wave from the details of a given scenario and acoustic environment. These waveforms have to account of all temporal and spatial environmental degradations associated with propagation from arbitrary sources, ambient noise, and reverberation in such environment. 
In a static environment the wave equation and, in particular, the Helmholtz equation [22] leads to a propagation solution in the frequency domain. The working in time domain is beneficial as temporal fluctuation and Doppler associated with moving boundaries can be included easily. Also this equation handles spatial coherence and the modal effects associated with low frequencies and shallow water [23]. The essence of simulation is to regard the caustic environment with time varying delays. This simulation was done with the help of FEMLAB and Matlab. In FEMLAB Helmholtz equation was solved to model acoustic wave propagation in water. Time which a wave takes to travel a particular distance was calculated by this model developed in FEMLAB. In Matlab transmission loss and ray tracing were used to solve these waves in time domain (obtained from FEMLAB) to simulate the actual working of Imagenex 881A profiling sonar.

The Helmholtz equation is given by

$$
\mathrm{d}_{\mathrm{a}} \frac{\partial \mathrm{u}}{\partial \mathrm{t}}-\Delta(\mathrm{c} \Delta \mathrm{u}+\alpha \mathrm{u}-\gamma)+\beta \Delta \mathrm{u}+\mathrm{au}=\mathrm{f}
$$

where $d_{a}$ is the mass coefficient.

$\mathrm{c}$ is the diffusion coefficient;

$\alpha$ is the conservative flux convection coefficient;

$\beta$ is the convention coefficient;

a is the absorption coefficient;

$\gamma$ is the conservative flux source;

$\mathrm{f}$ is the source term.

Here symbol $\Delta$ is the vector differential operator (gradient), defined as: 


$$
\Delta=\left(\frac{\partial}{\partial \mathrm{x}_{1}}, \ldots \ldots, \frac{\partial}{\partial \mathrm{x}_{\mathrm{n}}}\right)
$$

So $\Delta(\mathrm{c} \Delta \mathrm{u})$ means,

$$
\frac{\partial}{\partial \mathrm{x}_{1}}\left(\mathrm{c} \frac{\partial \mathrm{u}}{\partial \mathrm{x}_{1}}\right)+\ldots \ldots+\frac{\partial}{\partial \mathrm{x}_{\mathrm{n}}}\left(\mathrm{c} \frac{\partial \mathrm{u}}{\partial \mathrm{x}_{\mathrm{n}}}\right)
$$

Also $\beta \Delta \mathrm{u}$ means,

$$
\beta_{1} \frac{\partial \mathrm{u}}{\partial \mathrm{x}_{1}}+\ldots \ldots \ldots+\beta_{\mathrm{n}} \frac{\partial \mathrm{u}}{\partial \mathrm{x}_{\mathrm{n}}}
$$

In Equation $2.28 d_{a}$ represents a scalar or matrix for time-dependent systems, $\alpha, \beta$, and $\gamma$ are vectors with $\mathrm{n}$ components. The component $\mathrm{c}$ can be an $\mathrm{n} \times \mathrm{n}$ matrix to model anisotropic materials.

For our purpose time harmonic propagation of the wave equation was considered, so Helmholtz equation in time domain is given by following formula:

$$
-\Delta \cdot(\Delta \mathrm{u})+\mathrm{k}^{2} \mathrm{u}=0
$$

where $k=\frac{2 \pi}{\lambda}$

For reflection and diffraction at the boundaries Neumann boundary conditions were used. This is because Neumann conditions take into the account of multiple reflection from the surface. Neumann boundary condition used in this model is of the coefficient form and is given by following equations:

$$
\begin{aligned}
& n .(\Delta u)+i k u=2 i k, \text { inflow } \\
& n .(\Delta u)+i k u=0, \text { outflow }
\end{aligned}
$$


Inflow Equation 2.34 relates to the way at which a wave strikes the surface and Outflow relates to the way it is reflected back. In Neumann conditions the wave having highest intensity is only taken into account.

A model was generated to test the simulation of sonar. In this model a step function of 1.65 feet was created at the bottom with closed boundaries. Boundaries replicated the walls of the tank and step function indicated the solid-liquid interface inside a tank. Following results of simulation were obtained from the model generated in FEMLAB.

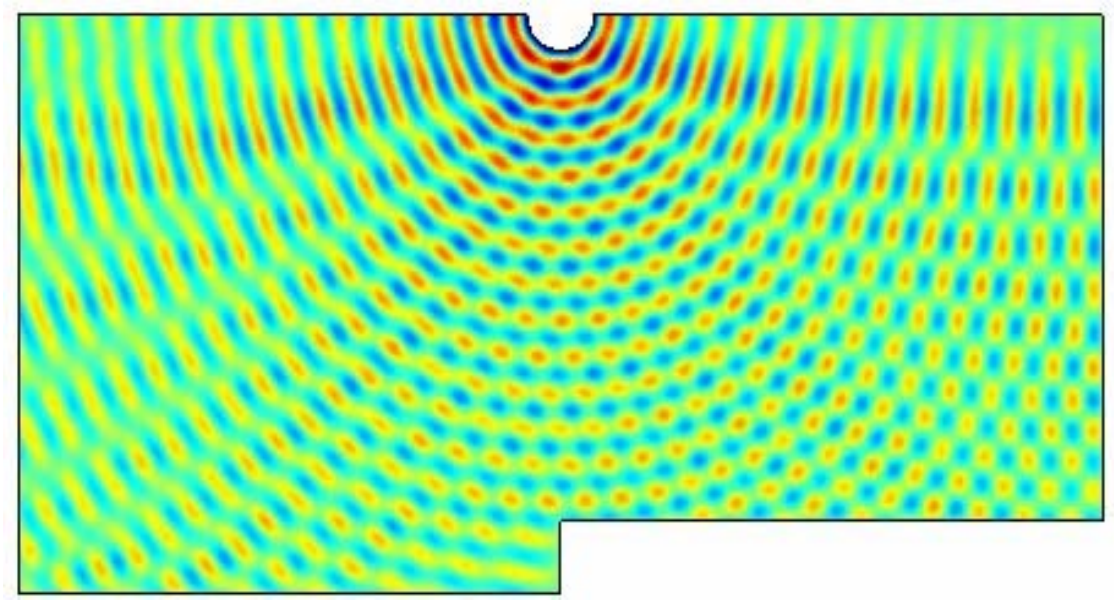

Figure 6: Surface propagation of a wave.

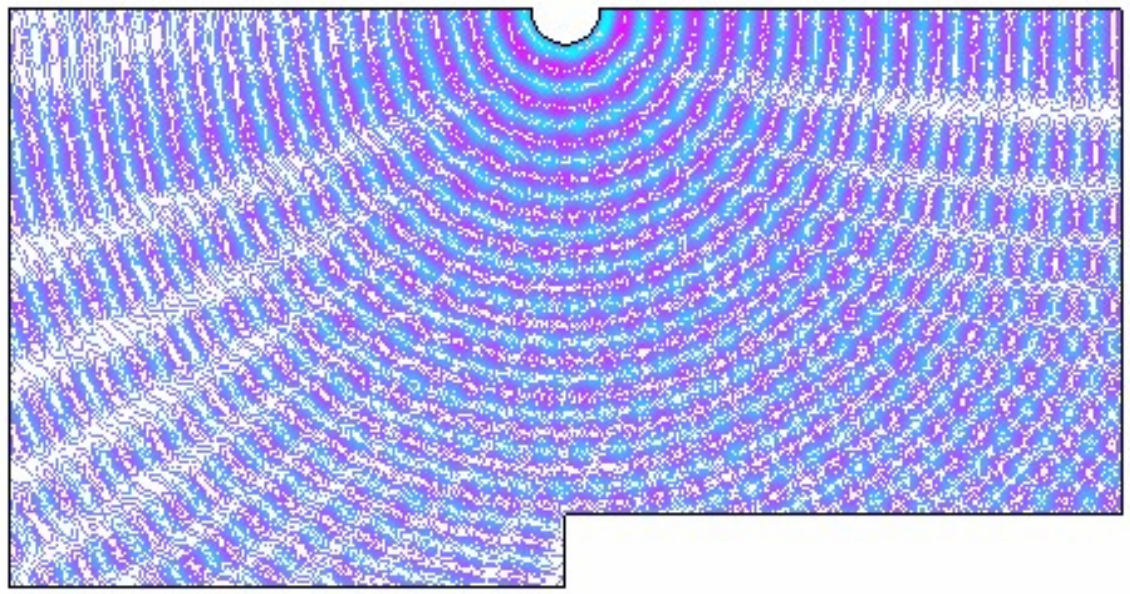

Figure 7: Wave propagation shown in contour plot. 


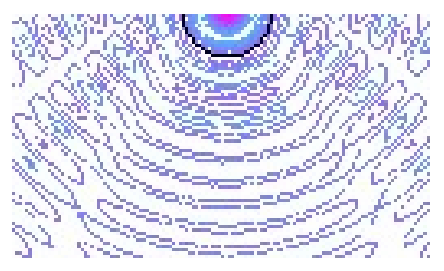

Figure 8: Enlarged image of wave contour plot also showing the conical shape of the wave.

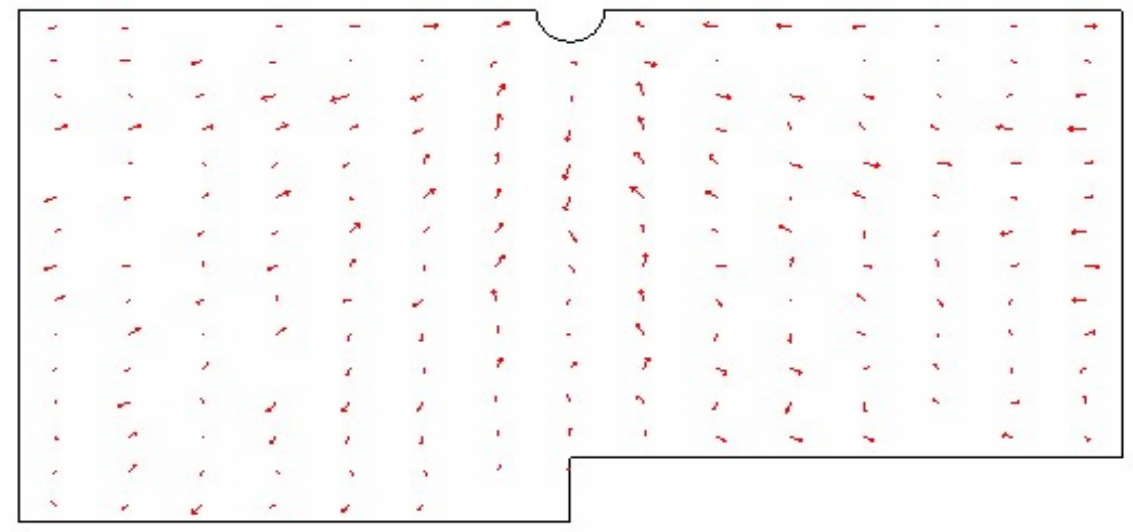

Figure 9: Wave propagation shown in arrow plot.

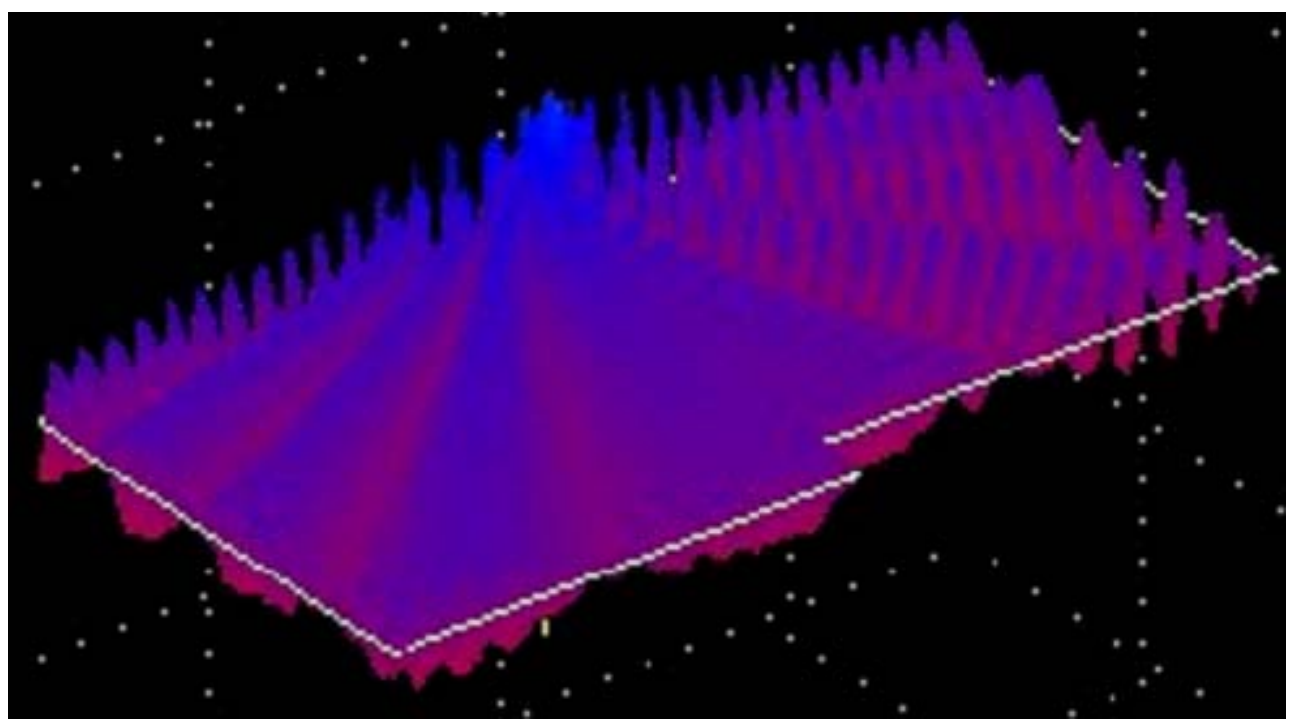

Figure 10: A 3-D representation of wave propagation. 
The images in Figures 6, 7, 9, and 10 shows the acoustic wave propagation in a tank filled with water. Time a wave takes to propagate to an abject and travel back to sonar transducer was recorded in this program. Figure 9 represents the arrow plot of the wave propagation in this figure the reflection and deflection of the wave can seen. Also Newman conditions are used so only the waves having highest intensity are shown. Figure 10 shows 3 -D solution of acoustic wave. Every $60^{\text {th }}$ wave is represented in the figure.The time obtained from these results were used as an input to the Matlab program which generated the sonar images. This program also took into account the transmission loss determined in the Table 4 .

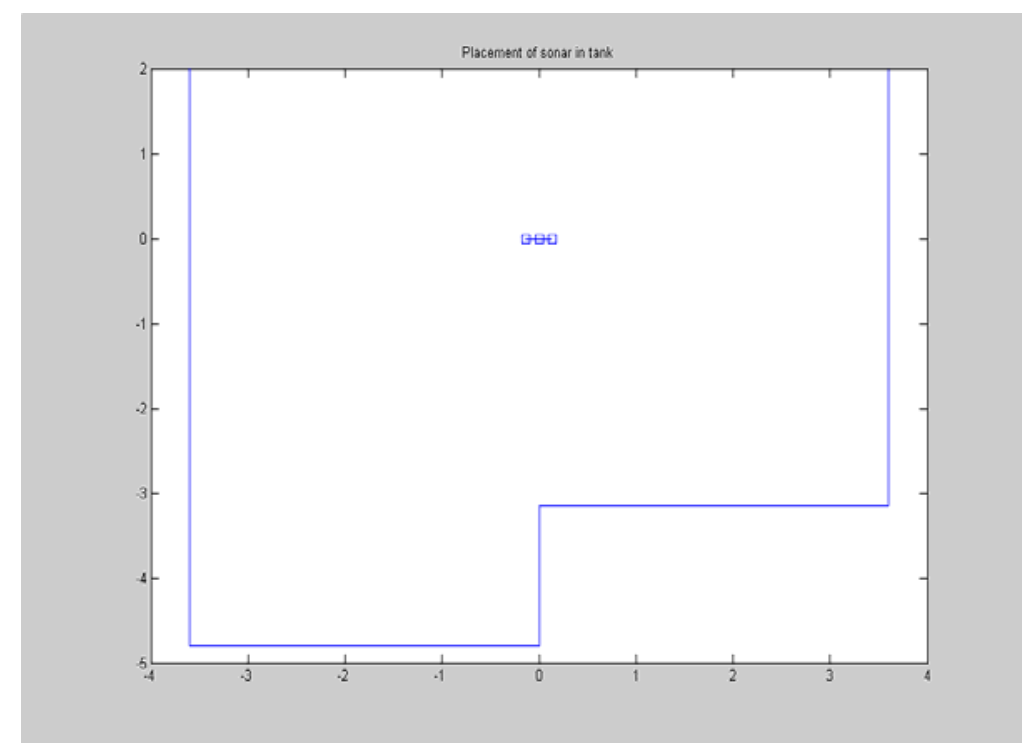

Figure 11: Sonar with actual position inside the tank.

Figure 11 shows the placement of sonar in the tank. Here the sonar is shown by square boxes (at the center of the image), representing sonar transducer. In this simulation transducer is considered to be rectangular rather than cylindrical. Also the arrangement shown is exactly same as of experiment 2 (describe in Chapter 4). 
Figure 12 was obtained as result of above arrangement by program written in the Matlab. The dots represent the position from where wave is reflected back from the surface. In this range was kept 6 feet and the total angle of sonar scan is $180^{\circ}$.

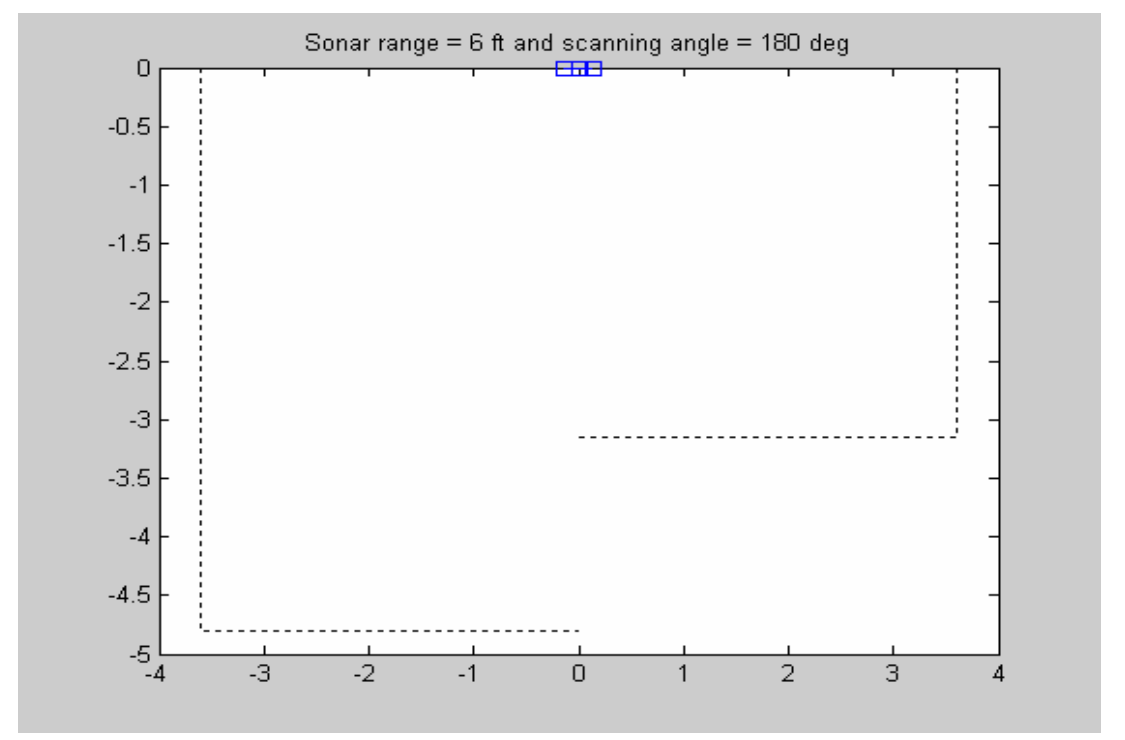

Figure 12: Image generated from the simulation when range is $6 \mathrm{ft}$.

In some cases multiple images of the solid-liquid interface was observed when sonar range was increased. These multiple images are called ghost images of the actual interface. The simulation also explained the phenomenon of generation of ghost images. The range in the above arrangement was increased from 6 feet to 15 feet to obtain ghost images.

Figure 13 shows the propagation of a single acoustic wave when range is 15 feet. It is seen that wave is reflected at multiple points before it reaches back to sonar. Sonar transducer determines the direction of this wave and also records the time which it took to travel back. When transducer generates a point in the direction of receiving wave by considering the time it has taken to travel back it plots it far from the actual point. 


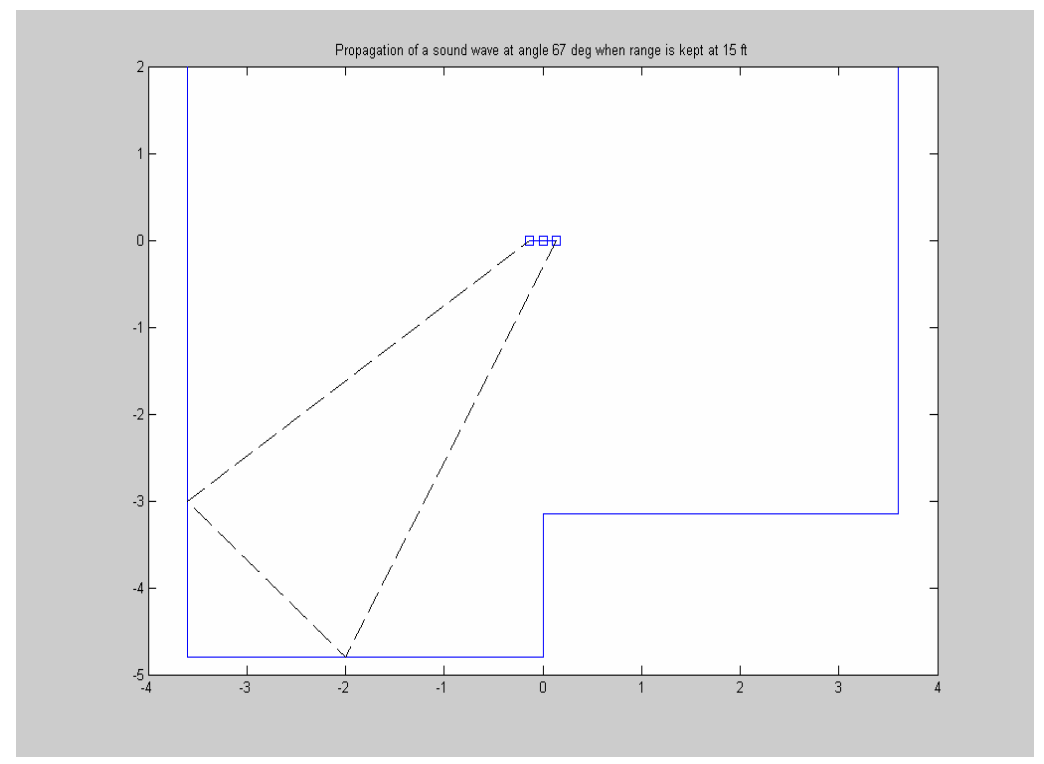

Figure 13: Propagation of a single wave incident at $67^{\circ}$ from the transducer and the range is kept 15 ft. instead of $6 \mathrm{ft}$.

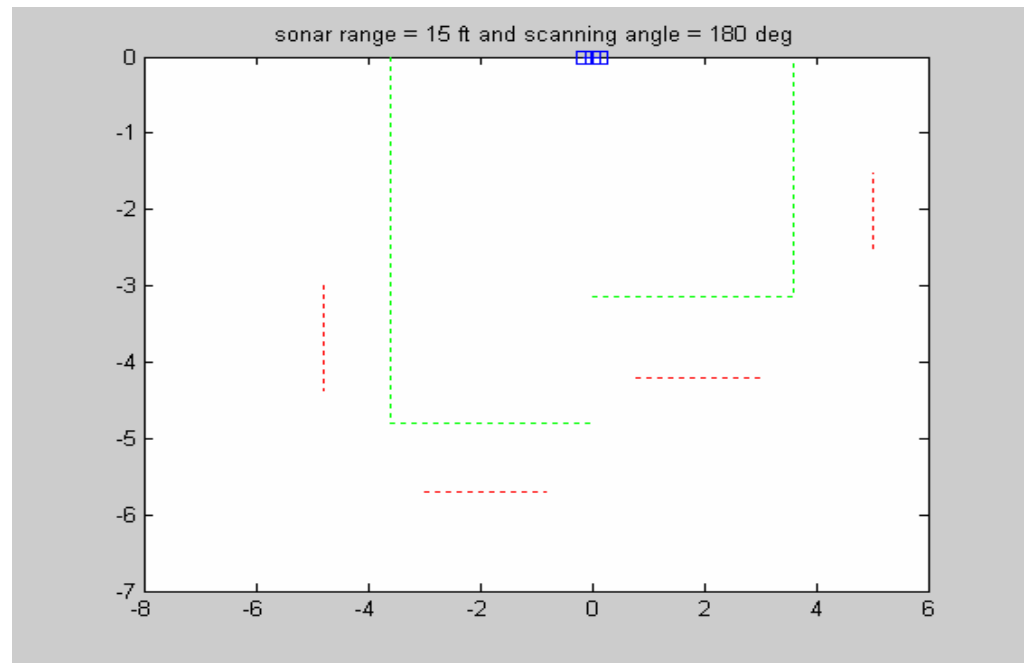

Figure 14: Image generated from simulation when range was kept $15 \mathrm{ft}$.

Figure 14 shows ghost image of the interface when sonar range is increased form

6 feet to 15 feet. Results obtained from this simulation were helpful in designing the experiments to demonstrate the efficacy of sonar in HLW tanks.

\section{Limitations of the simulation}

Following are the limitations of the simulation done: 
1. Consider transducer as rectangular;

2. Consider origin of the acoustic wave from a point;

3. Consider acoustic wave as a ray rather than conical wave.

\section{Unique Features of the simulation}

Following are the unique features of the simulation:

1. Takes transmission loss (calculated in Table 4) of water into account for propagating of acoustic wave;

2. Describe the origin of the ghost images;

3. Solve sonar equations for shallow water using both ray and wave technique;

4. Describe the working of sonar in close boundaries;

5. This simulation is valid for all the frequencies especially low frequencies;

6. Present solution which is easy to interpret;

7. It gives a complete solution. 


\section{Chapter 3}

\section{EXPERIMENTAL RESEARCH AND RESULTS}

\subsection{Summary of the seven sets of experiments conducted}

After studying the criterions for sonar deployment set forth by the Hanford site (discussed in Chapter 1) Imagenex 881A profiling sonar was selected based upon its ease for deployment (3.25 in. diameter and 9.75 in. long) through a 4-inch riser. In addition, its titanium and polyurethane body provides stability in caustic and radioactive environment such as in HLW tanks. To ascertain the potential of the Imagenex 881A profiling sonar inside HLW tanks several experiments were conducted. To design the experiments the conditions present inside HLW tanks were studied.

Hanford HLW tanks are typically filled with highly saturated caustic solutions $(\mathrm{pH}>14)$ that minimize corrosion due to radioactivity. The caustic nature of the solution causes low-density oxalate to form which may be similar in density to that of the caustic solution and remains suspended causing multiple layers of solids to form. These oxalates are formed because of the un-dissolved salts varying in size from microns to larger diameter that causes density variation between $1 \mathrm{~g} / \mathrm{cm}^{3}$ to $1.4 \mathrm{~g} / \mathrm{cm}^{3}$. Presence of tank wall and floor were also taken into account in designing experiments as any wall nearby to the sonar head may affect the sonar image due to multiple reflection leading to distorted image or ghost image. Another factor of consideration in the experimental design was re-suspension of solid particles due to pumping or mixing in HLW tanks. This

was again considered an important factor as scattered particles may affect propagation of 
sound wave in medium by reflecting or deflecting it different direction resulting in faulty readings.

Experiments 1 and 2 were designed to test the sonar's ability to accurately detect the solid-liquid interface in a tank of size $7 \mathrm{ft} .2$ in. diameter by $7 \mathrm{ft} .4$ in. height. An interface created by kaolin clay and water was imaged. Kaolin clay had $1 \mu \mathrm{m}$ diameter and $2.6 \mathrm{~g} / \mathrm{cm}^{3}$ density similar to the solid waste present in HLW tanks. Once sonar was able to map accurately the settled solid layer, Experiment 3 was designed to study the effect of the scattered solid particles present in the fluid on sonar images. The kaolin clay present in the tank was re-suspended with the help of submersible pump to simulate the scattered particles present in HLW tank during mixing or pumping.

After testing sonar for its accuracy, Experiment 4 was designed to test sonar's ability to detect solids having the similar density as of fluid. This experiment was performed in a fiber glass tank of size $5 \mathrm{ft} .8 \mathrm{in}$. by $2 \mathrm{ft} .11 \mathrm{in}$. with plastic beads placed in water. These plastic beads had density of $1.04 \mathrm{~g} / \mathrm{cm}^{3}$ which is only $4 \%$ more than the water density and simulated the light density oxalates present in HLW tanks.

To study the effect of tank wall and floor Experiment 5 was designed. Experiment 5 was performed in two phases. During the $1^{\text {st }}$ phase sonar was placed at various heights from the tank bottom and images were recorded. In the $2^{\text {nd }}$ phase a stainless steel metal plate ( $2 \mathrm{ft} . \times 2 \mathrm{ft}$.) was placed at certain distance from the sonar head and images were recorded. Images obtained from both phases were analyzed to determine an appropriate location of sonar in HLW tanks such that tank wall and floor do not interface in sonar working. 
After ascertaining the successful detection of solid-liquid interface in water the medium was changed to caustic solution. This was done to study the effect of caustic solutions on sonar imaging and measuring capabilities. Experiment 6 was designed to determine the life time of sonar in caustic solution, for this sonar was placed in a caustic solution having same constituents as that present in HLW tanks and was heated to $45^{\circ} \mathrm{C}$. The solution was heated to increase the reaction rate of the sonar hull (titanium and polyurethane) with caustic, if at all it reacts.

After making the sonar complete corrosion resistance, its performance in caustic solution was tested in Experiment 7. Experiment 7 was designed to image objects placed in varying density of caustic solutions. The density of the solution was varied to study its effect on the sonar measuring capabilities as speed of sound in a medium depends mainly on the density of the medium. In addition correct sound speeds for all the solutions were calculated to correct the sonar reading. The density of caustic solution was varied by mixing appropriate amount of sodium nitrate in the water. Sodium nitrate was chosen because of three reasons 1) its readily dissolves in water, 2) HLW tanks have large amounts of sodium nitrate in their caustic solution, 3) it increases the density of water from $1 \mathrm{~g} / \mathrm{cm}^{3}$ to $1.4 \mathrm{~g} / \mathrm{cm}^{3}$ which is the required range of density of caustic solution present in HLW tanks, and 4) it simulates the caustic solution present in HLW tanks. In general there were seven experiments performed.

1. To image various objects placed in a tank.

2. To obtain the accurate sonar measurements of solid layer heights and reference points inside the tank. Results were analyzed to determine the accuracy of the sonar. 
3. To obtain accurate sonar measurements of solid-liquid interface when light to vigorous mixing of the solids is occurring in the tank.

4. To demonstrate sonar's ability to detect light solids having similar density as of liquid.

5. To demonstrate ability of sonar to take accurate measurements with objects in near proximity to the sonar head.

6. To determine the chemical compatibility of sonar and cables to highly caustic solution $(\mathrm{pH}>14)$.

7. To analyze the performance of sonar in caustic solution. Data obtained from sonar was used to correct the sonar measurements for specific density and temperature.

\subsection{Experiment 1}

\subsubsection{Objective}

To image various objects placed in a tank.

\subsubsection{Setup}

This experiment was conducted to image objects placed inside $7 \mathrm{ft} .2$ in. diameter tank. Objects imaged were a metal drum, a metal plate, and a more complex shaped object. Also a gradient in a small plastic tank (3.5 ft in diameter ) was imaged.

\subsubsection{Detection of metal drum}

This experiment was performed in a metallic tank $(7 \mathrm{ft} .2$ in. diameter $x 7 \mathrm{ft} .4$ in. height) filled with water. A steel drum (1 ft. 6 in. diameter x $2 \mathrm{ft}$. 4in. height) was placed inside the tank first vertically then horizontally. 


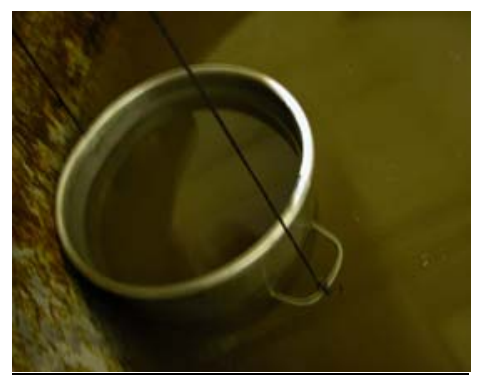

Results

Figure 15: Steel drum placed in metallic tank.

The image (Figure 16) of the arrangement shown in Figure 15 was generated when the sonar placed above drum. The two points marked as a and $\mathrm{b}$ in Figure 15 show the diameter of the drum when drum is placed vertically in the tank. From these points the height of the drum calculated is $2.329 \mathrm{ft}$.

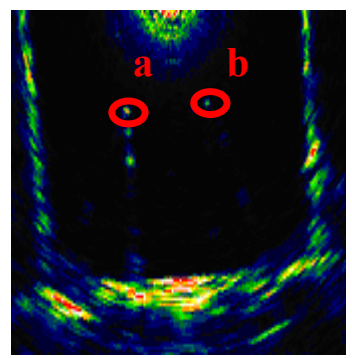

Figure 16: Image of the drum generated by the sonar when it is placed vertically in the tank.

Figure 17 shows image generated by the sonar when drum was placed horizontal to the bottom of the tank. The diameter of the drum was measured by using sonar software and was found to be $1.498 \mathrm{ft}$. The measurements obtained from the software show that the sonar is able to detect the object clearly with accurate measurements.

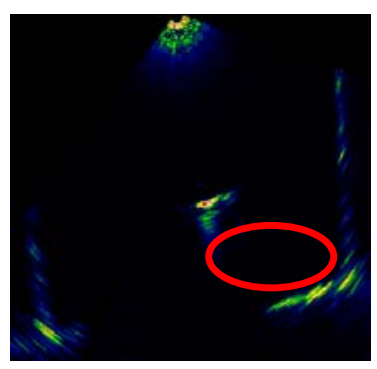

Figure 17: Image of the drum when it is placed horizontally in the tank. 


\subsubsection{Detection of metal objects}

This experiment used a square metal plate (2ft. $x$ 2ft.) hung with a simple mechanical arrangement. Figure 18 shows that there is a metal object placed on top of the metal plate being inserted into metallic tank of $7 \mathrm{ft} .2$ in. diameter $x 7 \mathrm{ft}$. 4 in. height.

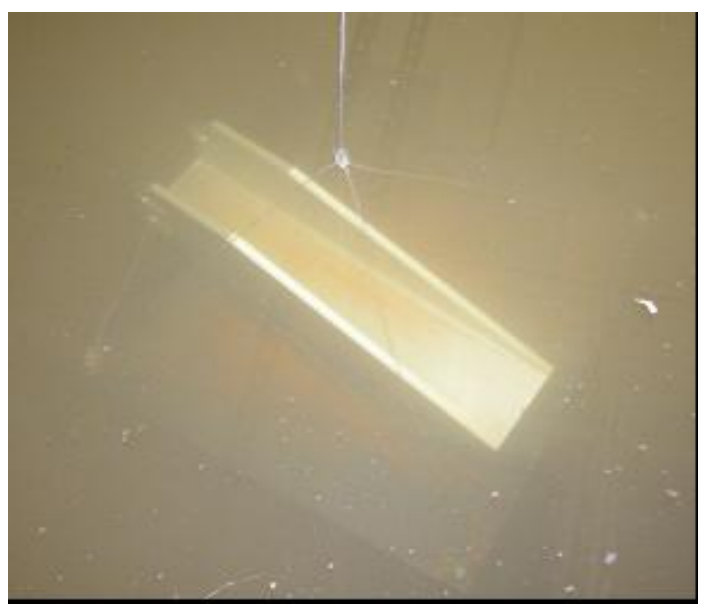

Figure 18: A square metal plate with metallic object placed on top of it.

Results

In Figure 19 "a" represents the knot on the wires used for suspension of metallic plate " $b$ " represents the metal plate with square object. The dimensions of the metal plate was calculated from sonar software was found to be $1.998 \mathrm{ft}$. The shape of the metal piece is also seen in the Figure 19.

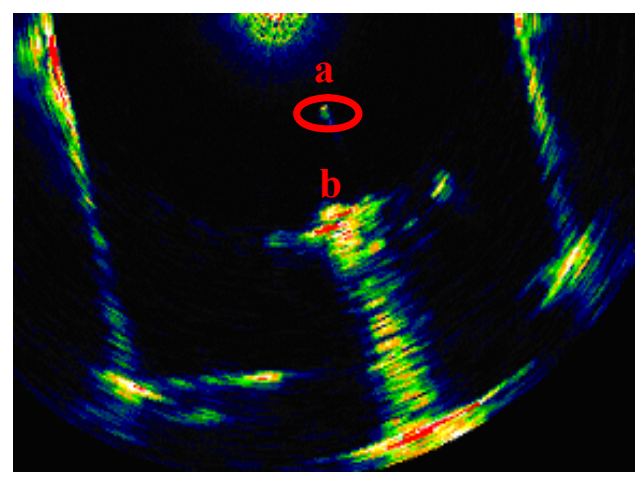

Figure 19: Image of the metal plate with a metallic object on top of it. 


\subsubsection{Detection of the solids layer gradient inside a tank}

The experiment was performed in a plastic drum of diameter $2 \mathrm{ft} .1$ in. diameter $\mathrm{x}$ $5 \mathrm{ft} .4$ in. height. Kaolin clay was used to create a gradient having slope $30^{\circ}$ inside this tank.

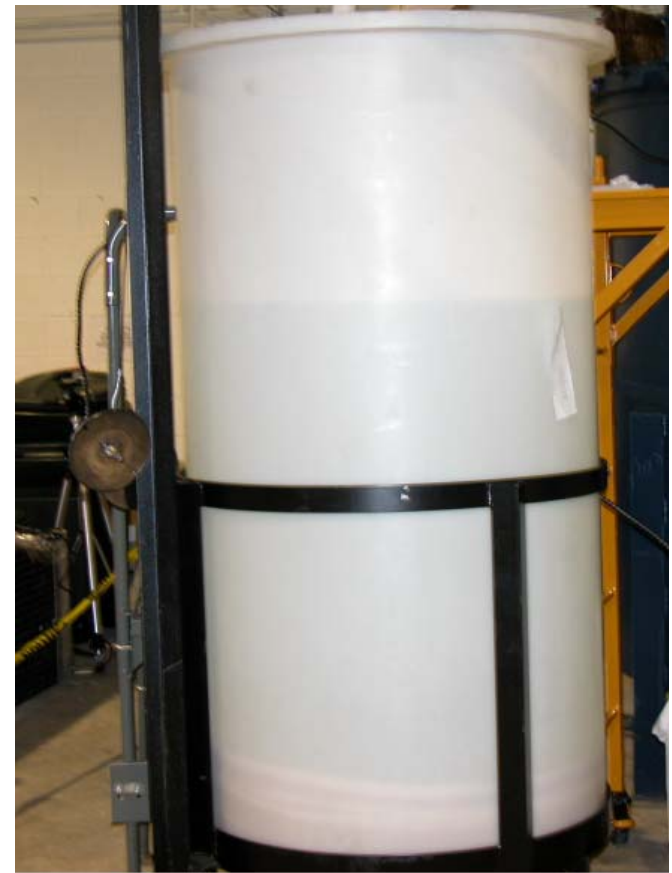

Figure 20: The gradient formed by kaolin clay inside the tank.

\section{Results}

The image of Figure 21 shows the gradient inside tank. The slope of the gradient was calculated and was found to be $30^{\circ}$.

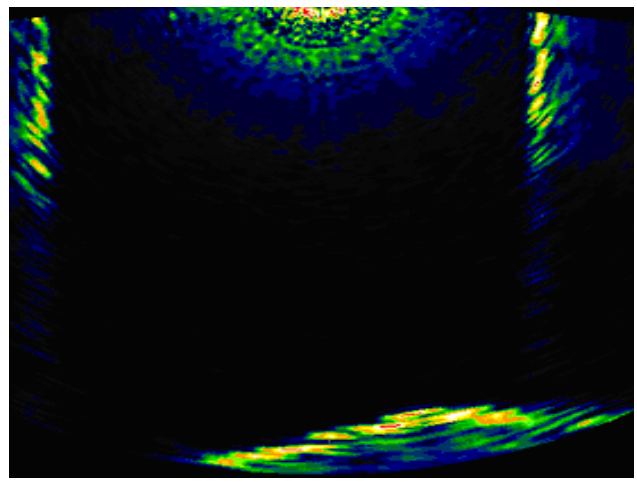

Figure 21: Gradient seen by sonar. 


\subsection{Experiment 2}

\subsubsection{Objective}

To obtain accurate sonar measurements for heights and distances between reference points inside tanks. This experiment was conducted to determine sonar accuracy in detecting solid-liquid interface. Measurements obtained from the sonar software were compared with the actual measurements.

\subsubsection{Setup}

This experiment was performed in a metallic tank (7 ft. 2 in. tank diameter $\mathrm{x} 7 \mathrm{ft}$. 4 in. height) filled with water. The bottom of the tank was divided into two half by a wooden barrier as shown in Figure 22. One side of the barrier was filled with kaolin clay $(500 \mathrm{lb})$ up to $1 \mathrm{ft} .3 .5 \mathrm{in}$. high. Other side of the barrier was left empty to create a step function. Water (approximately 1800 gal.) was filled in this tank.

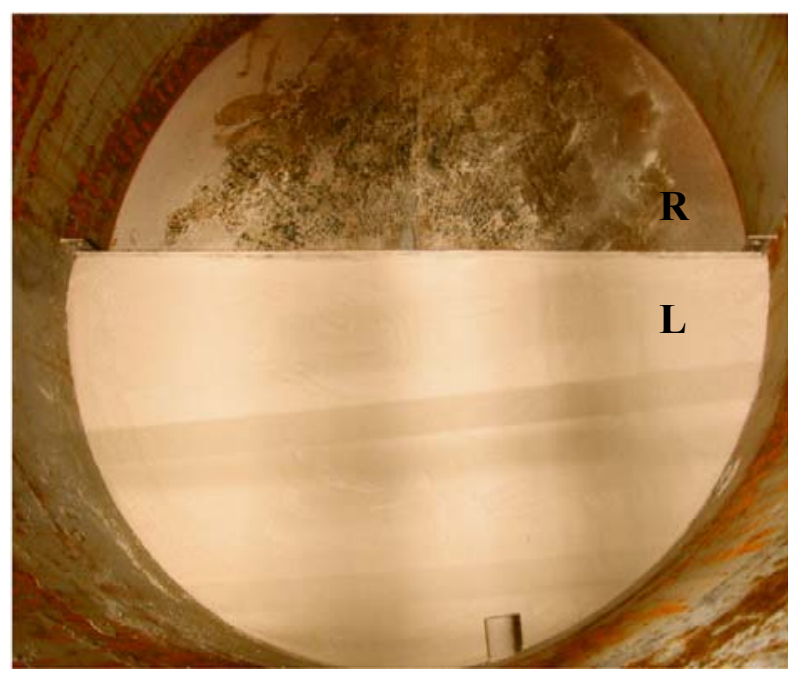

Figure 22: Top view of the interface created by kaolin clay and tank bottom.

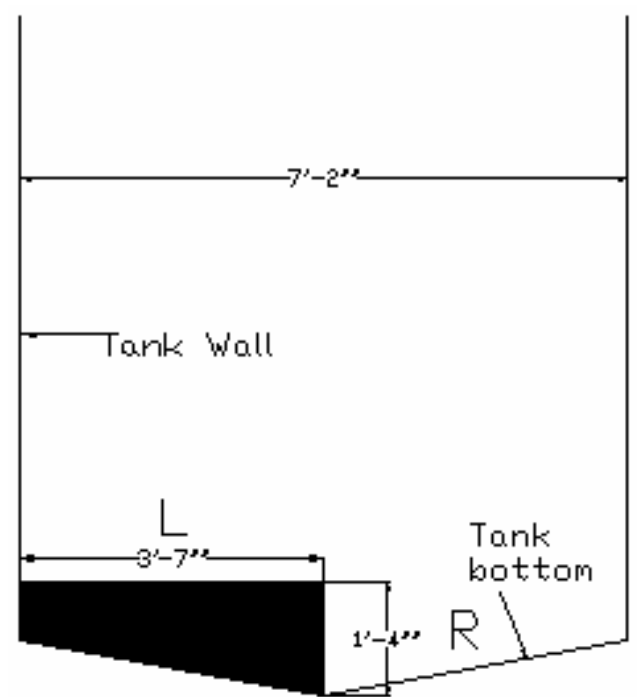

Figure 23: Side view of the tank showing the interface. 


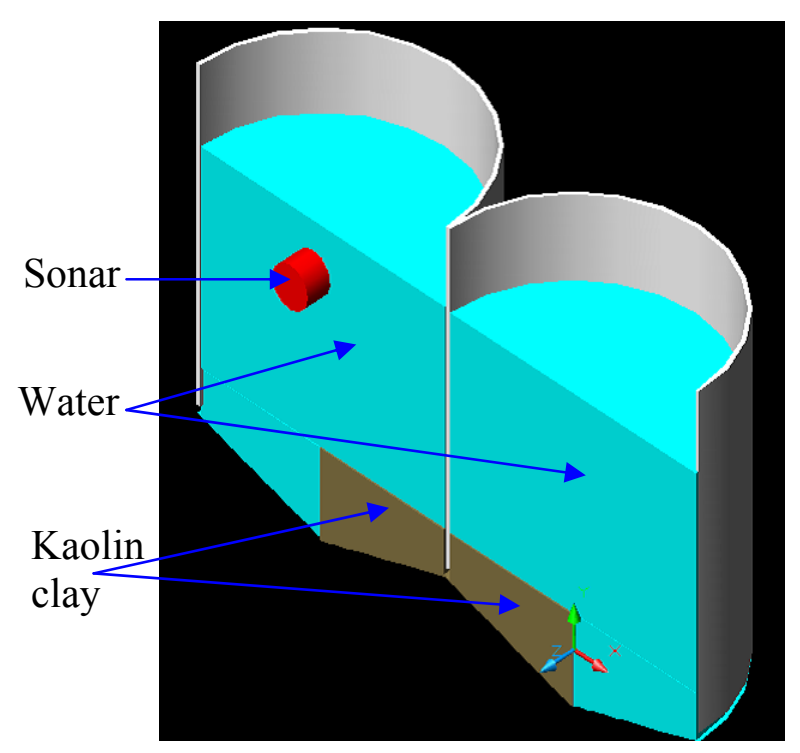

Figure 24: Cross section of the tank.

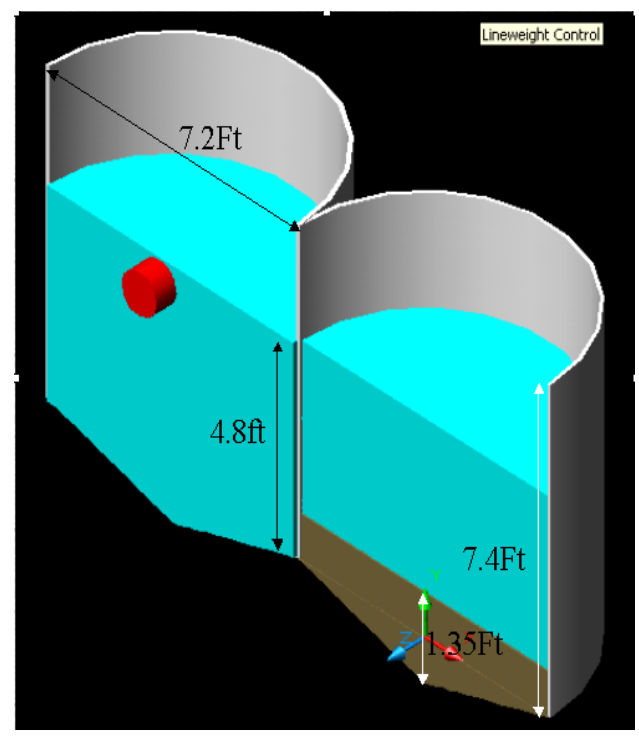

Figure 25: Cross section of the tank with dimensions.

In Figure 22 "L" represents the kaolin clay and " $\mathrm{R}$ " represents the empty tank bottom.

Sonar was suspended at $4 \mathrm{ft}$. 8in. height from bottom of the tank by the metal bars attached at the top of the tank. Sonar was suspended at a total of three different positions with three different angles as shown below in the Figure 26. 
Position1

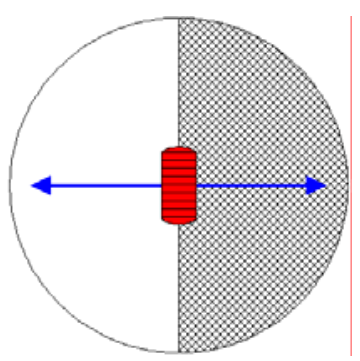

Position 4

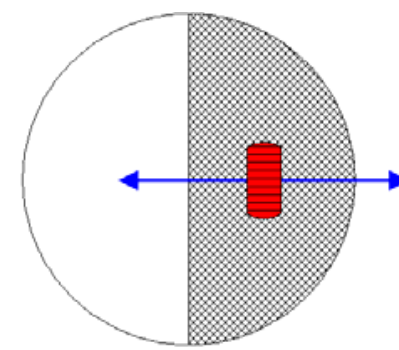

$\underline{\text { Position } 7}$
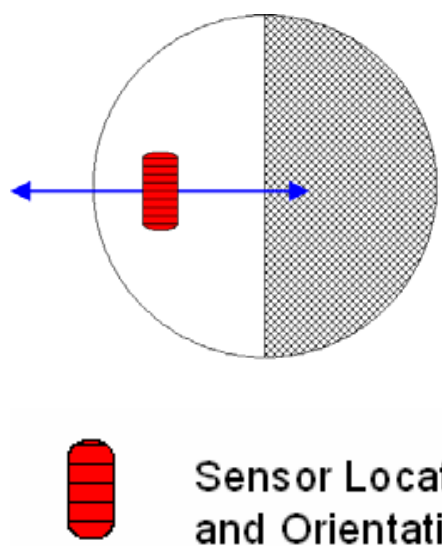

Sensor Location and Orientation
Position 2

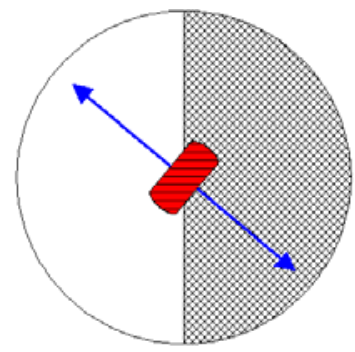

$\underline{\text { Position } 5}$

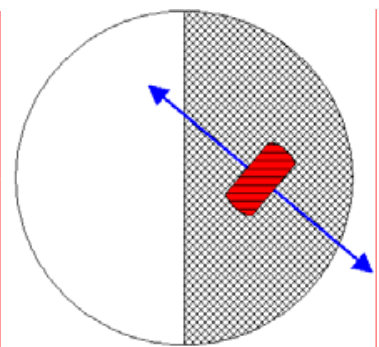

Position 8
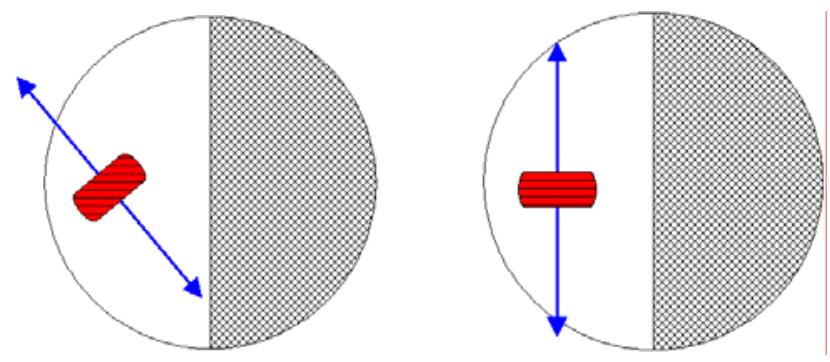

Imaging Plane

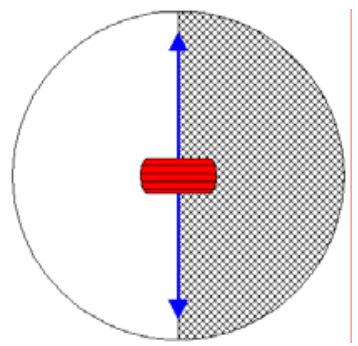

$\underline{\text { Position } 6}$

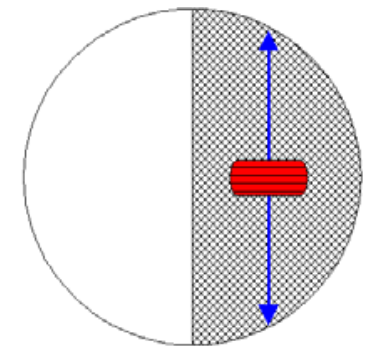

$\underline{\text { Position } 9}$

\section{Area showing the side of the tank filled with clay}

Figure 26: Different positions of the sonar where interface was imaged.

\subsubsection{Results}

Following images (Figures 27a to 27i) were obtained when sonar was suspended at nine different positions (shown in the Figure 26) in the tank from the top bars. The height of the sonar from tank bottom was kept the same for all the nine positions. 
$\underline{\text { Position1 }}$

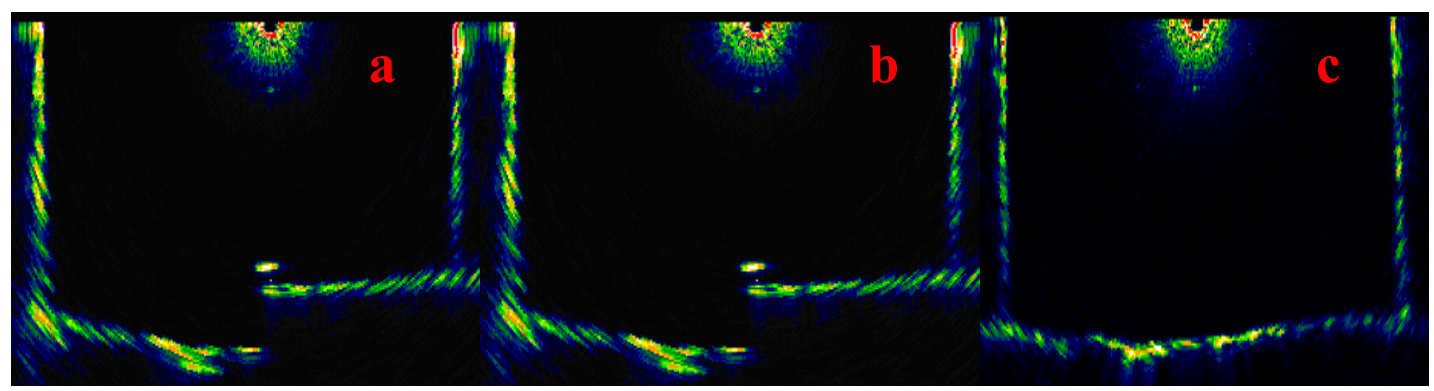

$\underline{\text { Position } 4}$

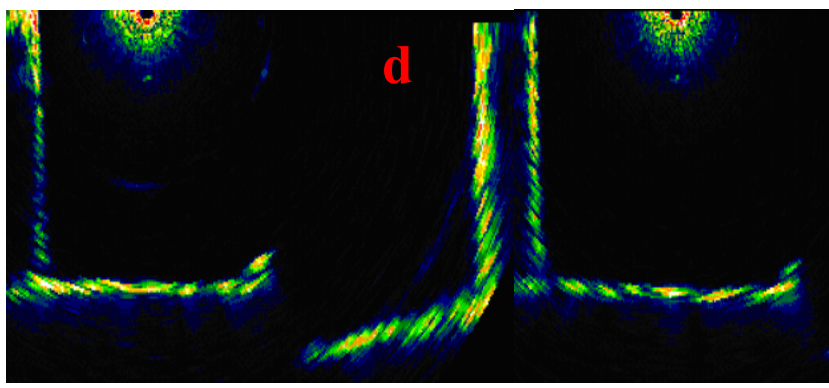

$\underline{\text { Position7 }}$

$\underline{\text { Position } 8}$

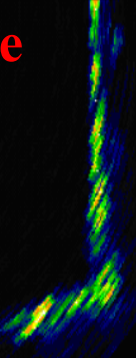

Nink

Posion 8
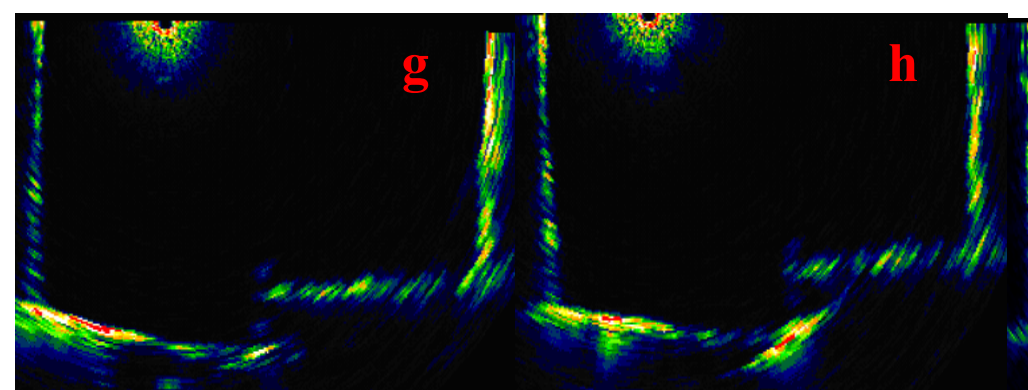

Figure 27: Images of interface generated by sonar software when sonar is suspended at different positions in the tank.

Here in Figure 27 "a" shows the image of the interface when sonar is at position 1, "b" shows the image of the interface when sonar is at position 2, "c" shows the image of the interface when sonar is at position 3, "d" shows the image of the interface when sonar is at position 4, "e" shows the image of the interface when sonar is at position 5, "f" shows the image of the interface when sonar is at position 6, "g" shows the image of the 
interface when sonar is at position 7, "h" shows the image of the interface when sonar is at position 8 and finally "i" shows the image of the interface when sonar is at position 9 .

\subsubsection{Analyses}

The actual and the observed (by sonar software) measurements, in addition to the relative error for the metal object are given in Table 4. Precise tape measurements were taken for the actual distances and were shown to be accurate within $1 \%$. Sound speed for determining the measurements of the objects by sonar was $1486 \mathrm{~m} / \mathrm{s}$ or $4875.3 \mathrm{ft} / \mathrm{s}$.

Table 4: Actual and Observed Measurements of the Interface and Sonar Heights.

\begin{tabular}{|c|c|c|c|c|c|c|}
\hline \multirow{2}{*}{$\begin{array}{c}\text { Position of } \\
\text { Sonar in } \\
\text { tank }\end{array}$} & $\begin{array}{c}\text { Actual } \\
(\mathrm{ft})\end{array}$ & $\begin{array}{c}\text { Observed } \\
(\text { Software }) \\
(\mathrm{ft})\end{array}$ & $\begin{array}{c}\text { Relative } \\
\text { Error }\end{array}$ & $\begin{array}{c}\text { Actual } \\
(\mathrm{ft})\end{array}$ & $\begin{array}{c}\text { Observed } \\
\text { (Software) } \\
(\mathrm{ft})\end{array}$ & $\begin{array}{c}\text { Relative } \\
\text { Error }\end{array}$ \\
\hline Position 1 & 5.90 & 5.891 & $0.2 \%$ & 1.37 & 1.369 & $0.2 \%$ \\
\hline Position 2 & 5.90 & 5.891 & $0.2 \%$ & 1.37 & 1.369 & $0.2 \%$ \\
\hline Position 3 & 5.90 & 5.890 & $0.2 \%$ & - & - & - \\
\hline Position 4 & 4.80 & 4.810 & $-0.2 \%$ & 0.93 & 0.931 & $-0.2 \%$ \\
\hline Position 5 & 4.80 & 4.810 & $-0.2 \%$ & 0.93 & 0.932 & $-0.2 \%$ \\
\hline Position 6 & 4.80 & 4.810 & $-0.2 \%$ & - & - & - \\
\hline Position 7 & 5.40 & 5.389 & $0.2 \%$ & 0.74 & 0.740 & $0.2 \%$ \\
\hline Position 8 & 5.40 & 5.389 & $0.2 \%$ & 0.74 & 0.741 & $0.2 \%$ \\
\hline Position 9 & 5.40 & 5.389 & $0.2 \%$ & - & - & - \\
\hline
\end{tabular}

The following bar graphs were plotted between actual and the observed values of the sonar heights (Figure 28) and the interface heights (Figure 29) measured when the sonar was suspended at 9 different positions in the tank. 


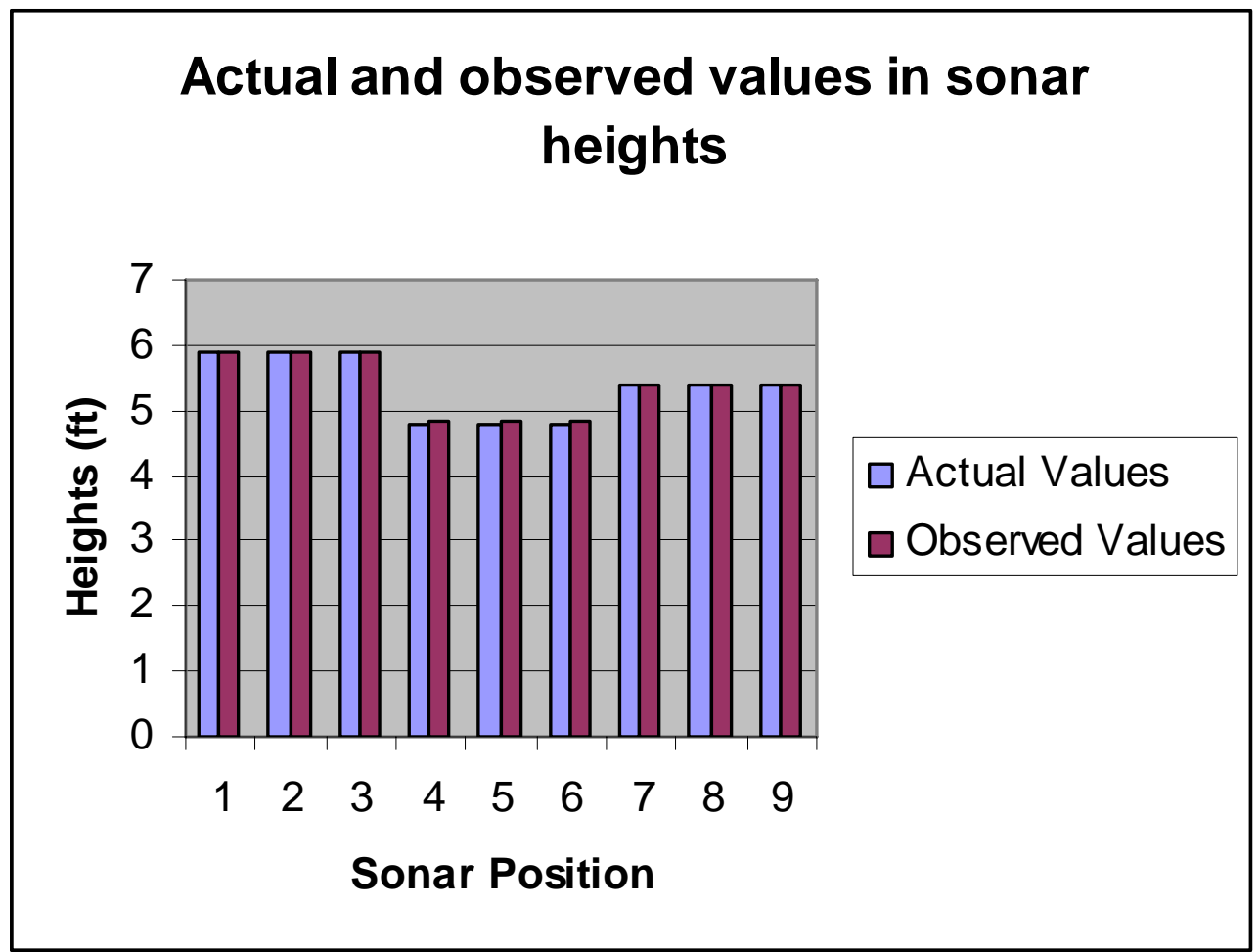

Figure 28: Bar plot of actual and observed values of the height of sonar from tank bottom at 9 different positions.

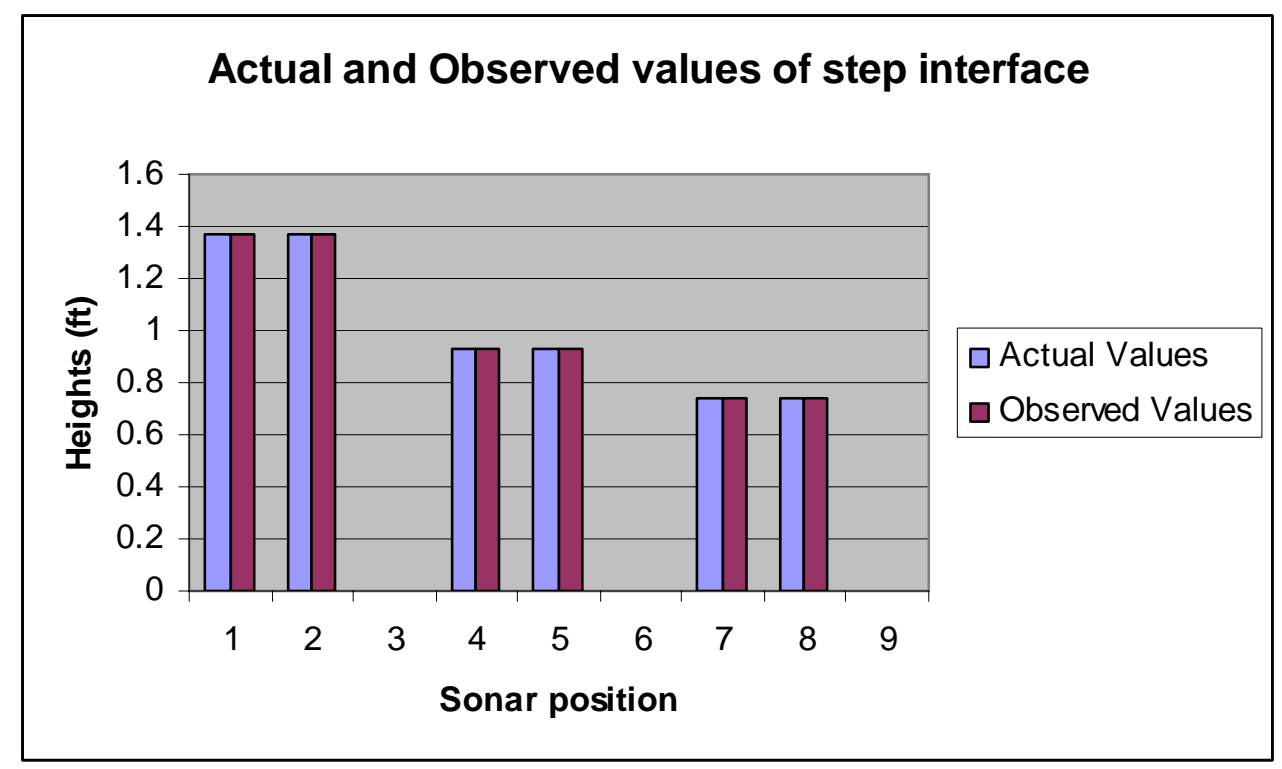

Figure 29: Bar plot of actual and observed values of the interface height when sonar is placed at 9 different positions. 


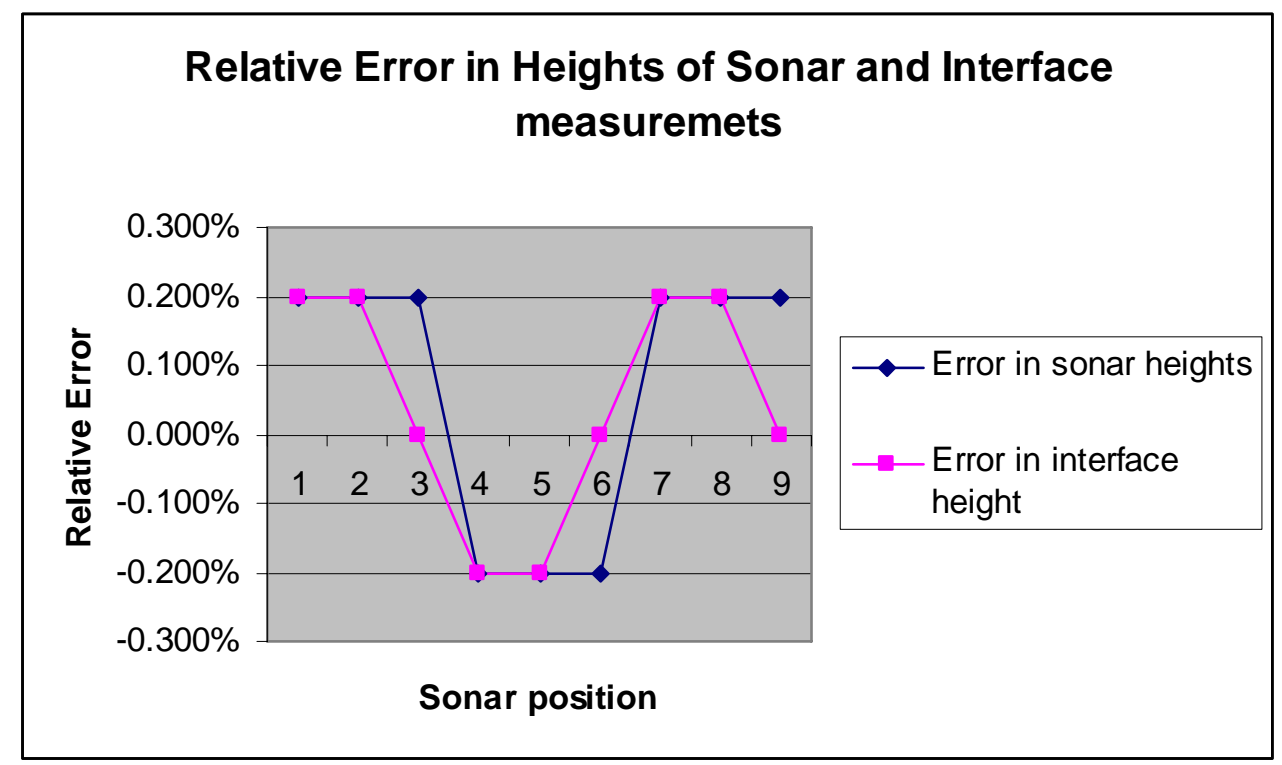

Figure 30: Plot of relative error in the sonar and interface height when sonar is placed at 9 different positions.

From the plot of Figure 30 it can seen that error in height of the sonar and interface is negligible. In the graph of Figure 30 there is negative value in relative error at positions 4,5 , and 6 . This is because the sonar software calculates the distances between of the reference points by dividing a shot (acoustic wave) in 250 parts, this generates an approximate values rather than accurate values. This approximate value can be more or less than the original value.

\subsection{Experiment 3}

\subsubsection{Objective}

To obtain accurate sonar measurements of solid-liquid interface during light to vigorous mixing of the solids in the tank. This his experiment was conducted to determine the accuracy of sonar in identifying the settled solid object shapes under intensive agitated conditions, with $30 \%$ solids (by weight) entrained in the liquid. 


\subsubsection{Setup}

The setup for this experiment was kept the same as of the last experiment (Experiment 2). Only a metal object (2 ft. 11 in. height $x 2 \mathrm{ft} .8$ in width) was placed on the empty side of the barrier in the tank (see Figure 31). Sonar was suspended at $4 \mathrm{ft}$. from the bottom of tank for the same nine position explained in Figure 26. Height of the sonar was kept constant for the experiment. A submersible pump was used to mix and agitate the water. Pump was rotated at a speed of $3450 \mathrm{rpm}$.

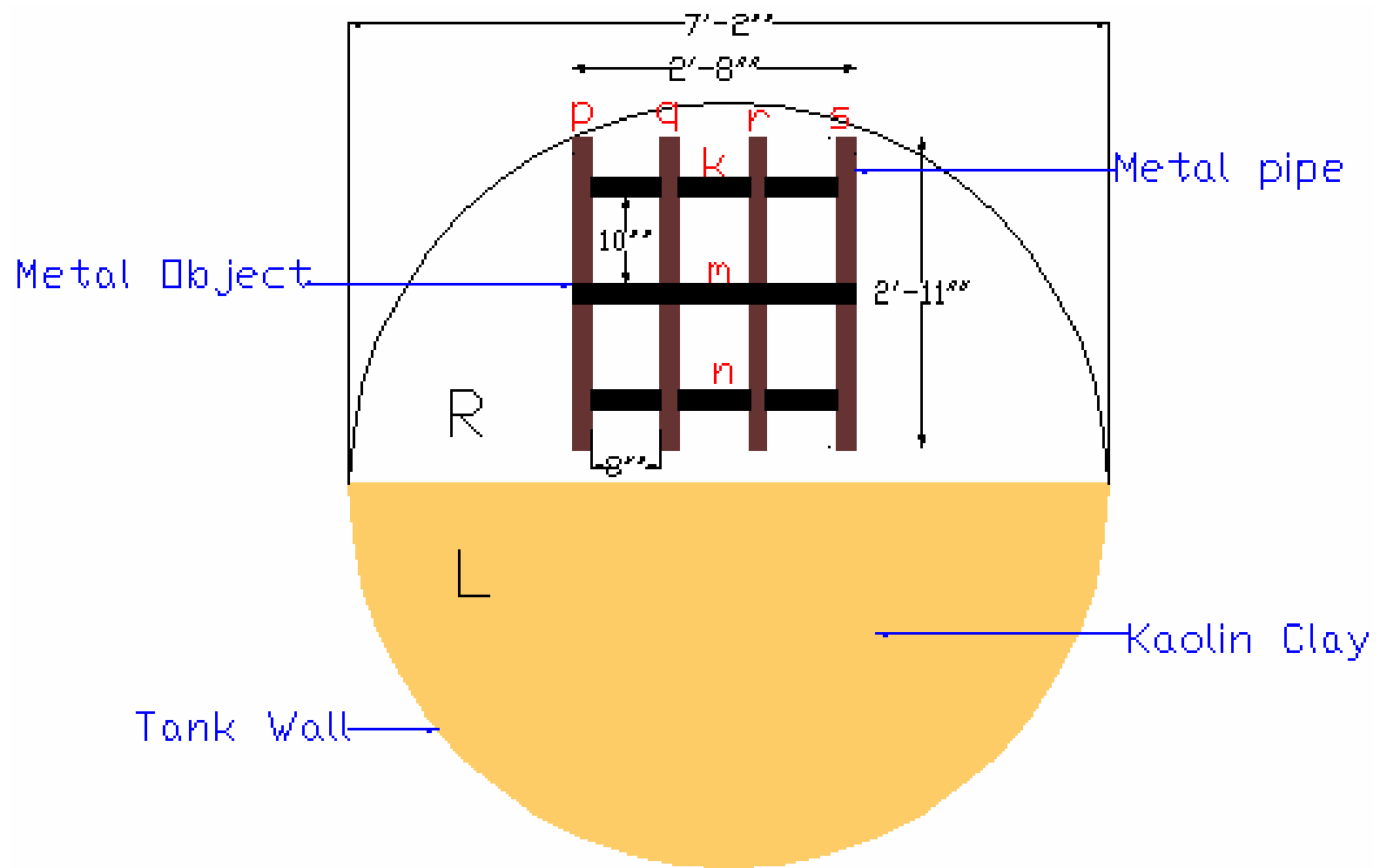

Figure 31: Top view of the tank showing the interface, the metal object in the tank.

In Figure 31 "L" represents the left half of the tank bottom with $1 \mathrm{ft} 35 \mathrm{in}$. kaolin clay and "R" represents the empty tank bottom. Also "p", "q", "r", "s", "k", “m” and "n" represents the different pipes in the metal object. 


\subsubsection{Results}

The amount of Kaolin clay suspended in the water during the agitation was measured at different speed of the submersible pump and it was found that $30 \%$ of kaolin clay by weight was suspended in the water.

The following images (Figures 32, 33, and 34) were obtained when sonar was held in positions 1, 2, and 3 (see Figure 26).

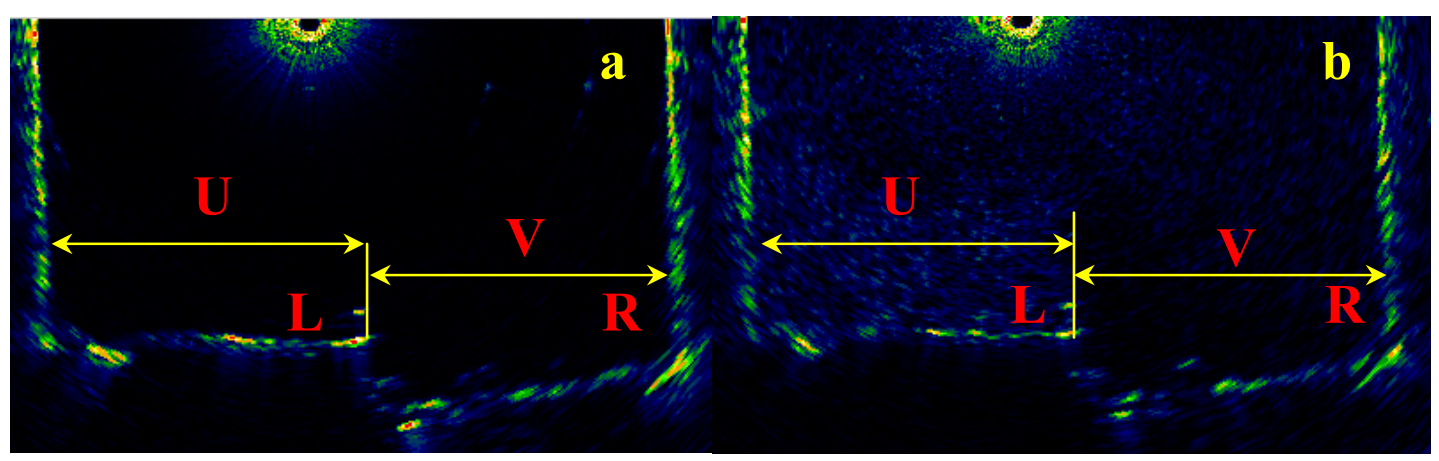

Figure 32: Interface generated when sonar is placed at position 1, (a) no agitation, and (b) during agitation.

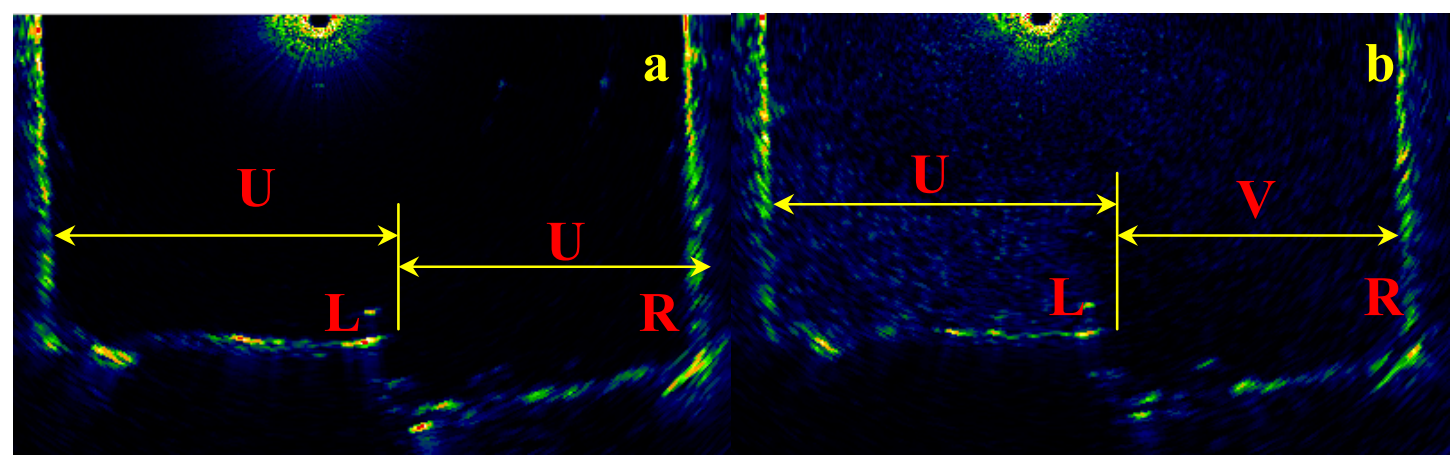

Figure 33: Interface generated when sonar is placed at position 2, (a) no agitation, and (b) during agitation. 


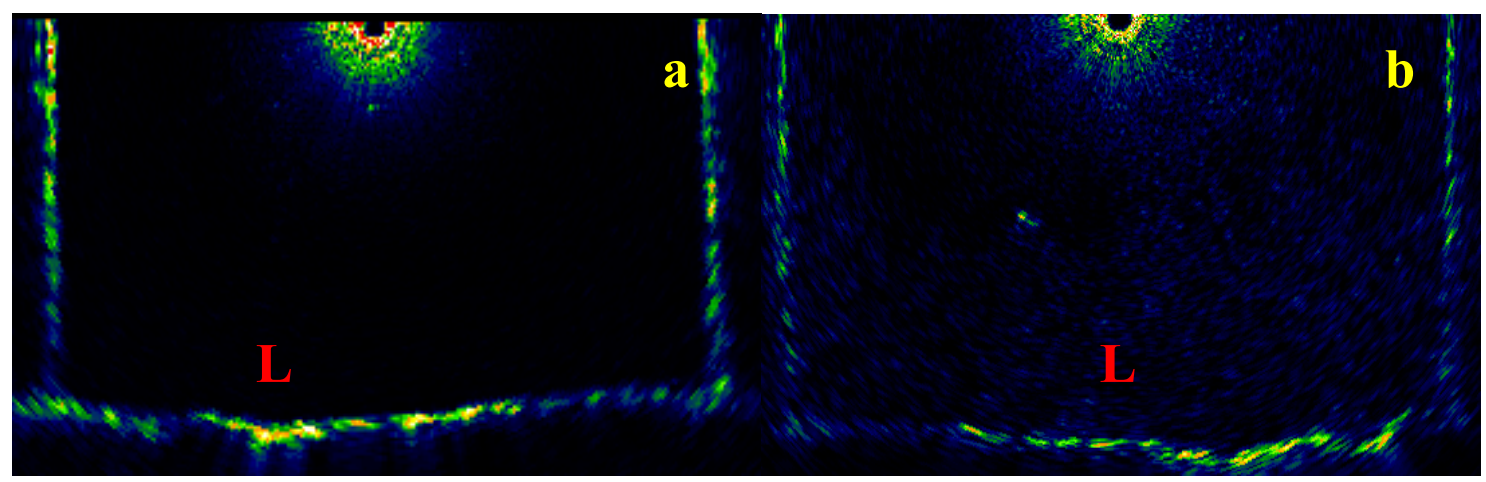

Figure 34: Image of the interface when sonar is placed at position 3 (a) no agitation, and (b) during agitation.

The following images (Figures 35, 36, and 37) were obtained when sonar was held in positions 4, 5, and 6 (see Figure 26).

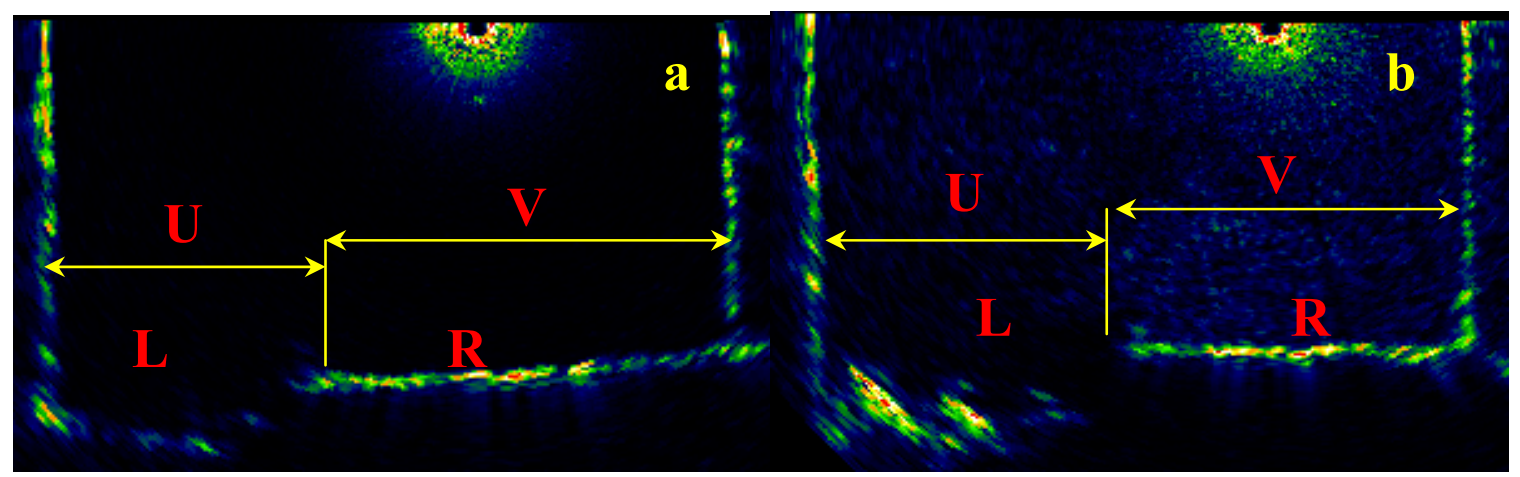

Figure 35: Interface generated when sonar is placed at position 4, (a) no agitation, and (b) during agitation.

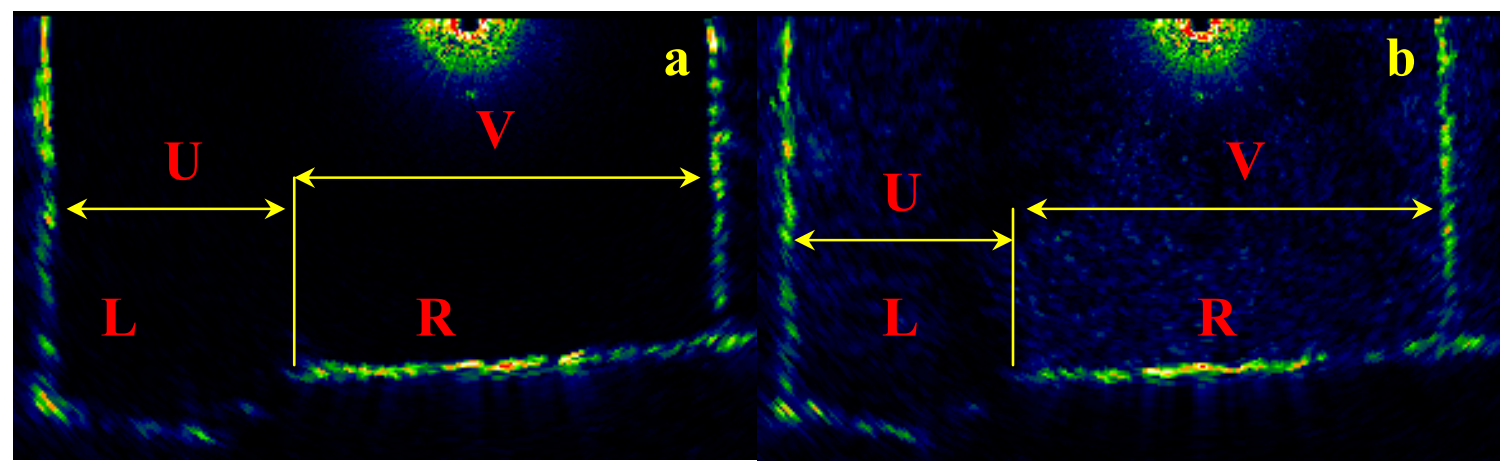

Figure 36: Interface generated when sonar is placed at position 5, (a) no agitation, and (b) during agitation. 


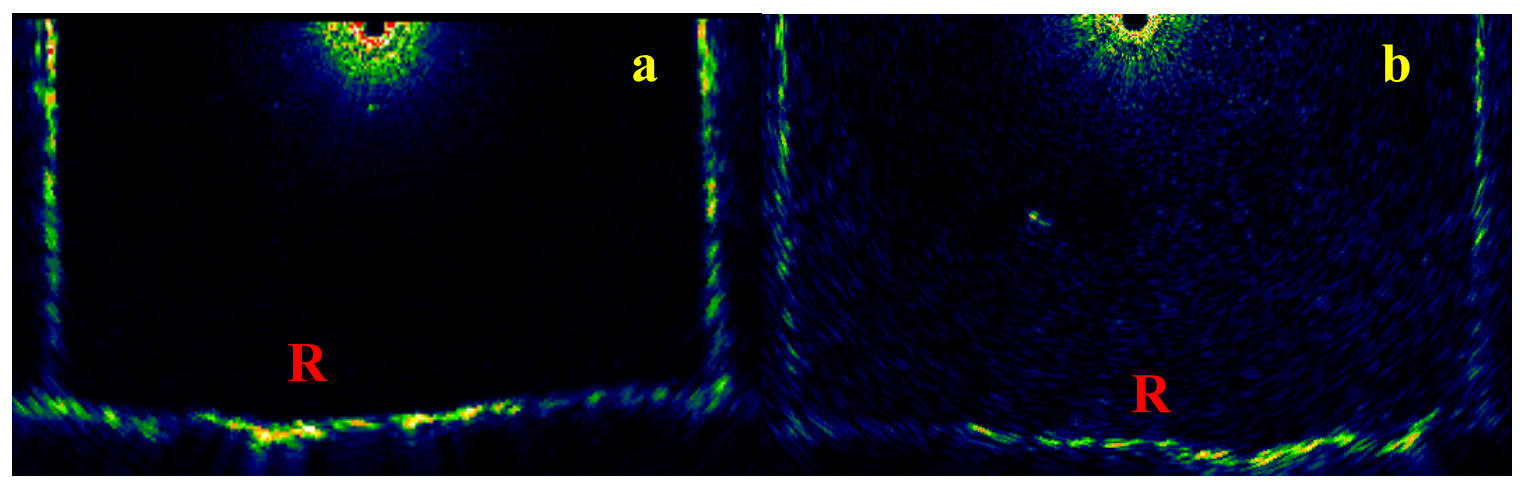

Figure 37: Interface generated when sonar is placed at position 6, (a) no agitation, and (b) during agitation.

The following images (Figure 38, 39, and 40) were obtained when sonar was held in positions 7, 8, and 9 (see Figure 26).

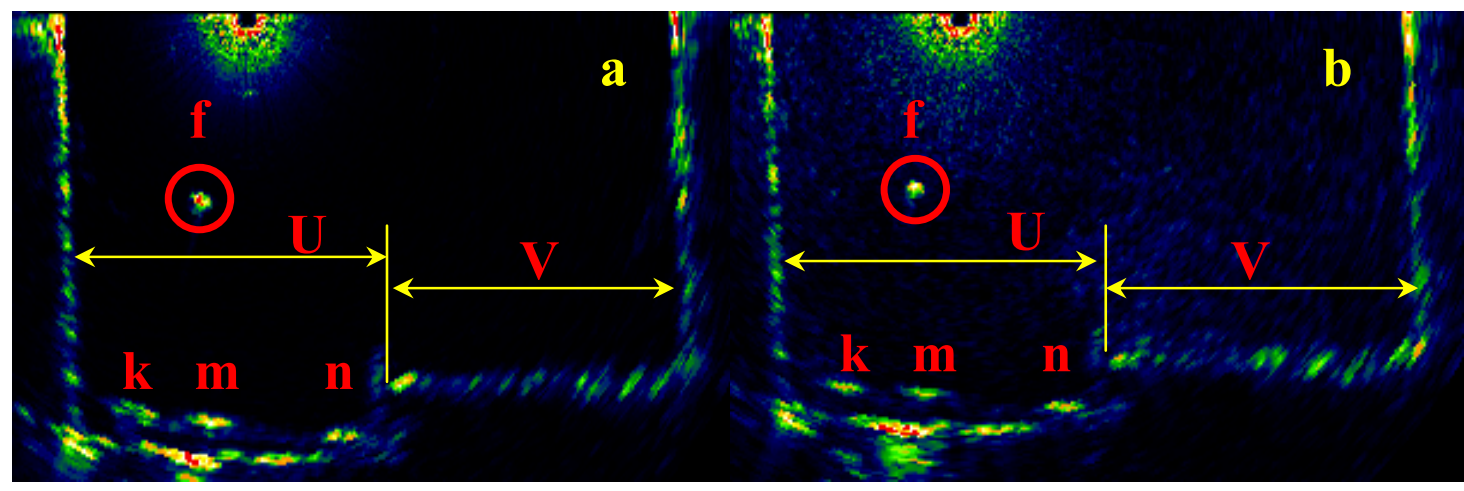

Figure 38: Interface generated when sonar is placed at position 7, (a) no agitation, and (b) during agitation.

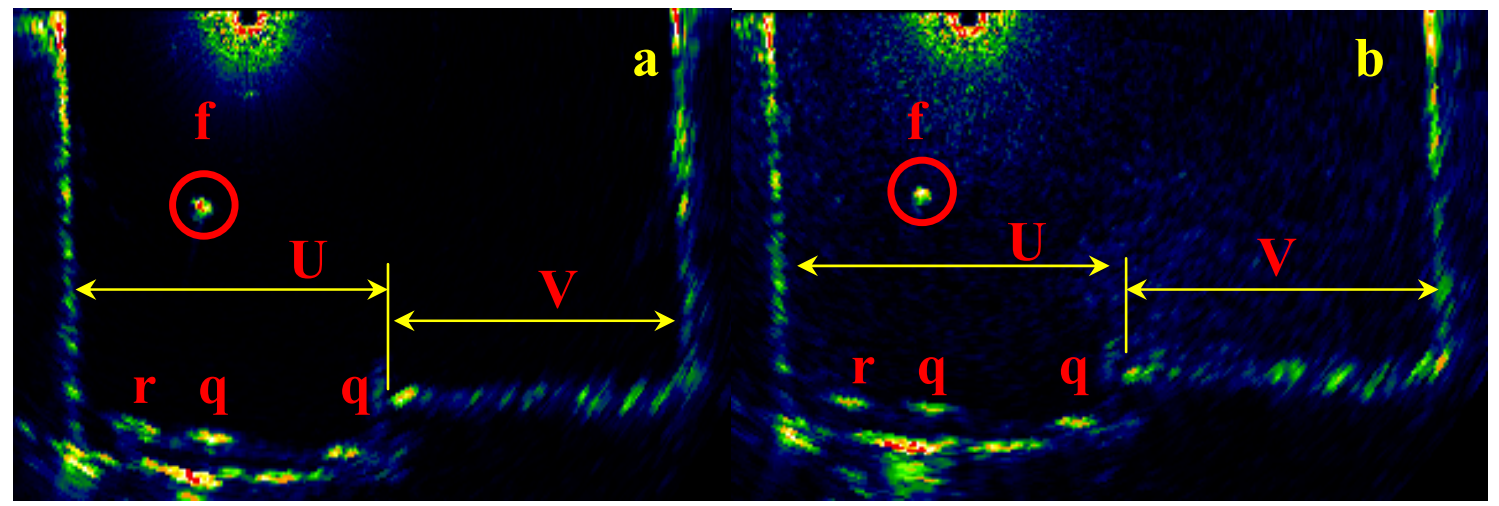

Figure 39: Interface generated when sonar is placed at position 8, (a) no agitation, and (b) during agitation. 


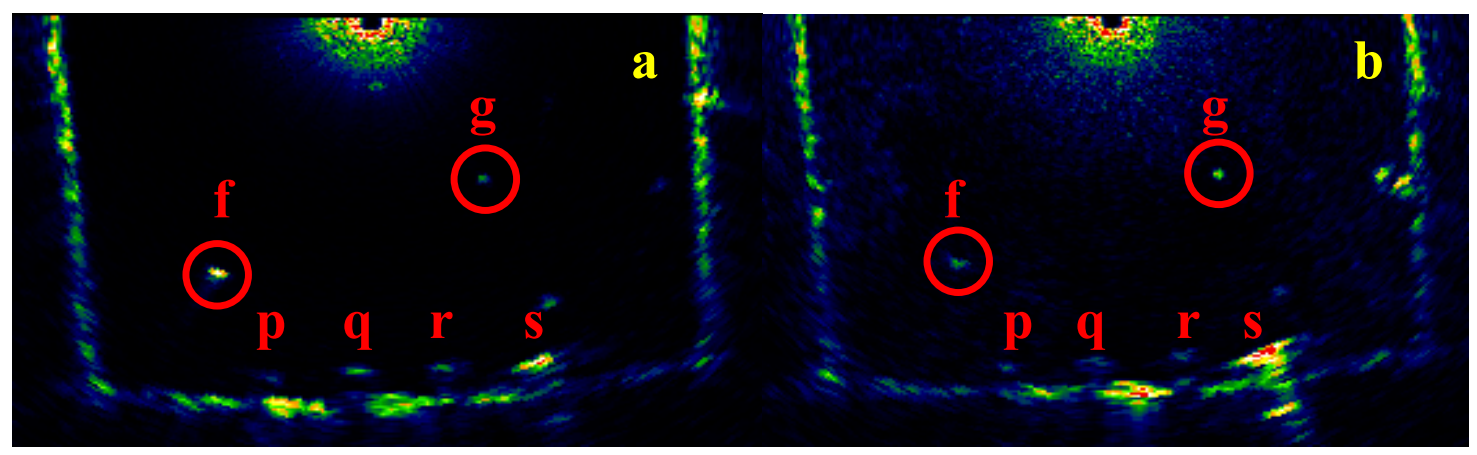

Figure 40: Interface generated when sonar is placed at position 9, (a) no agitation, and (b) during agitation.

The significant contiguous area of reflection points "p", “q", "r" ,"s", "k", “m” and "n" represent the 2-D image of the metal object, where "p" corresponds to the first pipe (Figure 23) in the metal object and "q" corresponds to the second pipe and so forth. The two significant reflecting points " $\mathrm{f}$ " and "g" correspond to the knots on the wire through which the metal object was tied.

\subsubsection{Analyses}

The actual and the observed (by sonar software) measurements, in addition to the relative error for the metal object are shown in Tables 5 and 6 . When the sonar was placed at the center of tank, the distance between the kaolin clay and the tank wall (U) was similar to that of the clay and the tank wall (V) with and without agitation (Figures 29a and 29b) Precise tape measurements were taken for the actual distances were and shown to be accurate within $1 \%$. Sound speed for determining the measurements of the objects by sonar was $1486 \mathrm{~m} / \mathrm{s}$ or $4875.3 \mathrm{ft} / \mathrm{s}$. 
Table 5: Actual and Observed Values of the Interface Height Before and During Agitation.

\begin{tabular}{|c|c|c|c|c|c|c|c|}
\hline & \multicolumn{3}{|c|}{$\mathrm{U}$} & \multicolumn{3}{|c|}{$\mathrm{V}$} \\
\hline & & $\begin{array}{l}\text { Actual } \\
\text { (ft) }\end{array}$ & $\begin{array}{l}\text { Observed } \\
(\mathrm{ft})\end{array}$ & $\begin{array}{l}\text { Relative } \\
\text { Error }\end{array}$ & $\begin{array}{l}\text { Actual } \\
\text { (ft) }\end{array}$ & $\begin{array}{l}\text { Observed } \\
(\mathrm{ft})\end{array}$ & $\begin{array}{l}\text { Relative } \\
\text { Error }\end{array}$ \\
\hline \multirow{2}{*}{$\begin{array}{l}\text { Position } \\
1\end{array}$} & No agitation & 3.60 & 3.593 & $0.2 \%$ & 3.60 & 3.593 & $0.1 \%$ \\
\hline & $\begin{array}{l}\text { During } \\
\text { agitation }\end{array}$ & 3.60 & 3.593 & $0.2 \%$ & 3.60 & 3.594 & $0.1 \%$ \\
\hline \multirow[b]{2}{*}{$\begin{array}{l}\text { Position } \\
2\end{array}$} & No agitation & 3.60 & 3.598 & $0.5 \%$ & 3.60 & 3.60 & $0.0 \%$ \\
\hline & $\begin{array}{l}\text { During } \\
\text { agitation }\end{array}$ & 3.60 & 3.592 & $0.2 \%$ & 3.60 & 3.598 & $0.0 \%$ \\
\hline \multirow[b]{2}{*}{$\begin{array}{l}\text { Position } \\
3\end{array}$} & No agitation & 7.20 & 7.194 & $0.0 \%$ & - & - & - \\
\hline & $\begin{array}{l}\text { During } \\
\text { agitation }\end{array}$ & 7.20 & 7.188 & $0.1 \%$ & - & - & - \\
\hline \multirow[b]{2}{*}{$\begin{array}{l}\text { Position } \\
4\end{array}$} & No agitation & 4.48 & 4.478 & $0.0 \%$ & 2.60 & 2.598 & $0.0 \%$ \\
\hline & $\begin{array}{l}\text { During } \\
\text { agitation }\end{array}$ & 4.48 & 4.476 & $0.0 \%$ & 2.60 & 2.596 & $0.0 \%$ \\
\hline \multirow[b]{2}{*}{$\begin{array}{l}\text { Position } \\
5\end{array}$} & No agitation & 2.60 & 2.60 & $0.0 \%$ & 4.48 & 4.483 & $0.0 \%$ \\
\hline & $\begin{array}{l}\text { During } \\
\text { agitation }\end{array}$ & 2.60 & 2.589 & $0.4 \%$ & 4.48 & 4.484 & $0.0 \%$ \\
\hline \multirow[b]{2}{*}{$\begin{array}{l}\text { Position } \\
6\end{array}$} & No agitation & - & - & - & 6.40 & 6.389 & $0.0 \%$ \\
\hline & $\begin{array}{l}\text { During } \\
\text { agitation }\end{array}$ & - & - & - & 6.40 & 6.391 & $0.0 \%$ \\
\hline \multirow{2}{*}{$\begin{array}{l}\text { Position } \\
7\end{array}$} & No agitation & 4.08 & 4.079 & $0.0 \%$ & 3.26 & 3.262 & $0.0 \%$ \\
\hline & $\begin{array}{l}\text { During } \\
\text { agitation }\end{array}$ & 4.08 & 4.077 & $0.0 \%$ & 3.26 & 3.267 & $0.0 \%$ \\
\hline \multirow[b]{2}{*}{$\begin{array}{l}\text { Position } \\
8\end{array}$} & No agitation & 3.26 & 3.260 & $0.0 \%$ & 4.08 & 4.086 & $0.0 \%$ \\
\hline & $\begin{array}{l}\text { During } \\
\text { agitation }\end{array}$ & 3.26 & 3.258 & $0.0 \%$ & 4.08 & 4.087 & $0.0 \%$ \\
\hline
\end{tabular}

The bar plot of Figure 41 shows the actual and the observed distance between the tank wall and the interface. 


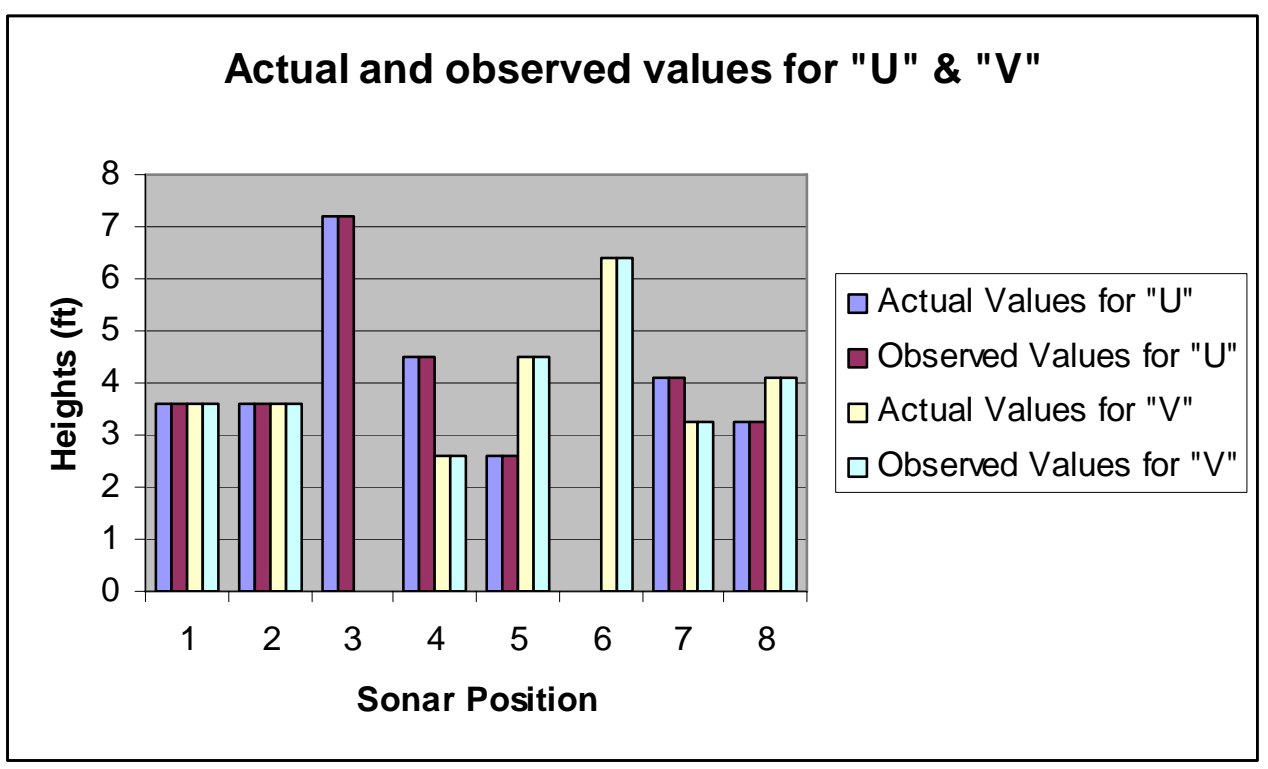

Figure 41: Bar plot of the actual and observed values of the distance between the tank wall and the interface.

Table 6: Actual and Observed Measurements of the Metal Object with Relative Error.

\begin{tabular}{|l|l|l|l|l|l|l|l|}
\hline \multicolumn{2}{|c|}{} & \multicolumn{3}{|l|}{ Distance between p and q } & \multicolumn{2}{l|}{ Distance between q and r } \\
\cline { 3 - 9 } & $\begin{array}{l}\text { Actual } \\
(\mathrm{ft})\end{array}$ & $\begin{array}{l}\text { Observed } \\
(\mathrm{ft})\end{array}$ & $\begin{array}{l}\text { Relative } \\
\text { Error }\end{array}$ & $\begin{array}{l}\text { Actual } \\
(\mathrm{ft})\end{array}$ & $\begin{array}{l}\text { Observ } \\
\text { ed (ft) }\end{array}$ & $\begin{array}{l}\text { Relative } \\
\text { Error }\end{array}$ \\
\hline \multirow{2}{*}{$\begin{array}{l}\text { Position } \\
9\end{array}$} & $\begin{array}{l}\text { No } \\
\text { agitation }\end{array}$ & 0.66 & 0.660 & $0.0 \%$ & 0.66 & 0.662 & $-0.0 \%$ \\
\cline { 2 - 9 } & $\begin{array}{l}\text { During } \\
\text { agitation }\end{array}$ & 0.66 & 0.658 & $0.0 \%$ & 0.66 & 0.658 & $0.3 \%$ \\
\hline \multirow{2}{*}{$\begin{array}{l}\text { Position } \\
9\end{array}$} & $\begin{array}{l}\text { No } \\
\text { agitation }\end{array}$ & 0.66 & 0.660 & $0.0 \%$ & 2.91 & 2.910 & $0.0 \%$ \\
\cline { 2 - 9 } & $\begin{array}{l}\text { During } \\
\text { agitation }\end{array}$ & 0.66 & 0.659 & $0.0 \%$ & 2.91 & 2.899 & $0.0 \%$ \\
\hline \multirow{2}{*}{$\begin{array}{l}\text { Position } \\
9\end{array}$} & $\begin{array}{l}\text { No } \\
\text { agitation }\end{array}$ & 4.00 & 4.000 & $0.0 \%$ & - & - & - \\
\cline { 2 - 10 } & $\begin{array}{l}\text { During } \\
\text { agitation }\end{array}$ & 4.00 & 4.002 & $-0.0 \%$ & - & - & - \\
\hline
\end{tabular}




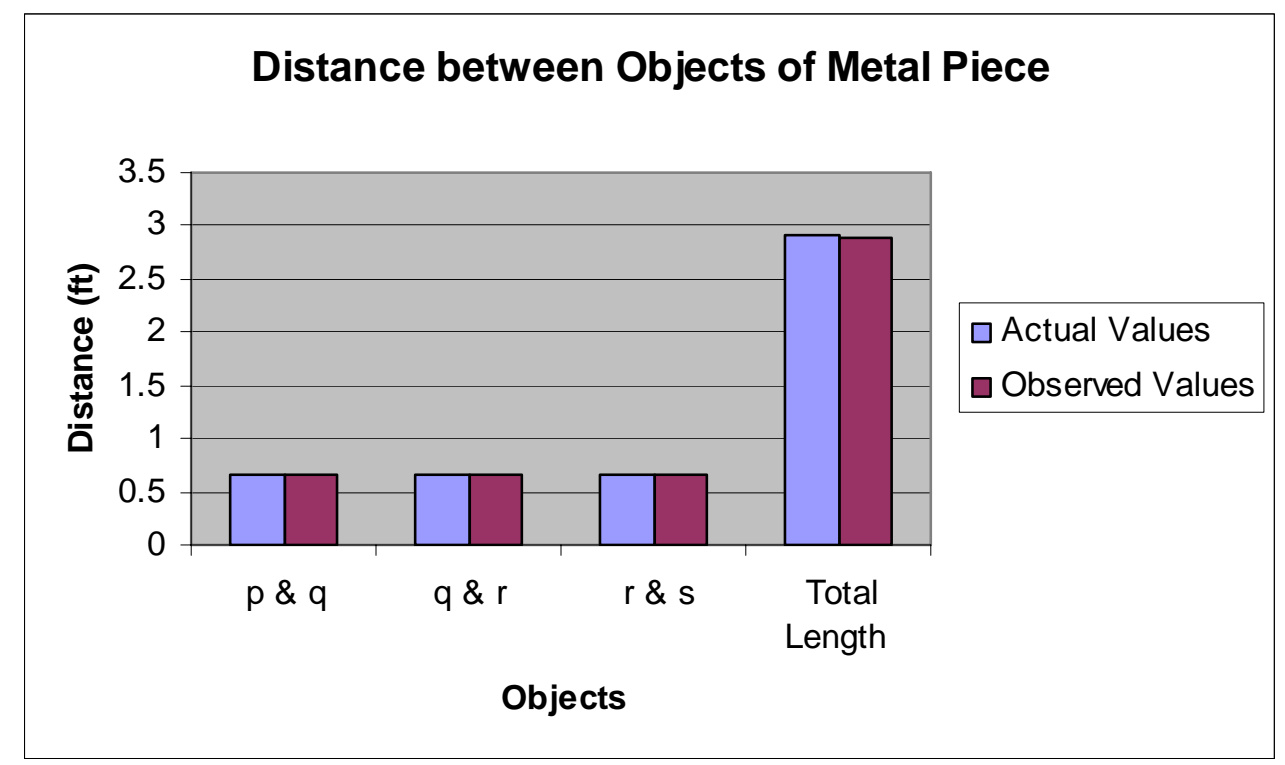

Figure 42: Bar plot of the actual and the observed distance between the different pipes of the metal object when the sonar is at position 9.

Form the plots of Figure 41 and 42 it can be seen that profiling sonar can detect the solidliquid interface accurately even when $30 \%$ solids (by weight) are present in the liquid. However there is generation of ghost images in the images obtained from sonar software. This is because of agitation and solids present in water. Because of the solids the sound waves are scattered in different direction leading to the multiple reflections from single point. Ghost images causes error in the measurements as they not easy to distinguish them from the original images.

\subsection{Experiment 4}

\subsubsection{Objective}

To determine the ability of the sonar to image the interface of light settled solids (density within $4 \%$ of the liquid above the solids). This experiment was conducted to test the ability of sonar to image the upper most surface of settled solids layer having a similar density to that of the fluid. The ability to detect less dense solids is important in 
retrieving HLW since these solids settled last and can clog waste transfer lines if pumps lowered near to this upper most layer.

\subsubsection{Setup}

This experiment was performed in a fiberglass tank of 70 in. diameter $x 33$ in. high. The bottom of the tank was divided into two equal halves by a barrier (I-Beam). On one side of the barrier, three geometric shapes: a square- and two rectangular- shaped metal pieces were filled with plastic beads to a height of 4 in. (Figures 43 and 44). The remaining portion on this side (where geometric shapes were placed) was filled with plastic beads to a height of 2 inches. On the other side of the barrier, a rectangular-shaped piece and one right triangular-shaped piece were filled with plastic beads to a height of 4 in. Water was then added to the tank. The sonar was suspended from the top of the tank by a metal rod fixed to the sidewall of the tank and positioned at a $62^{\circ}$ angle with respect to the barrier. Sonar was suspended at a height of $24 \mathrm{in}$. from the bottom of the tank. Sonar was suspended at two different positions one center of the tank and one near to the tank wall to verify the results of the experiment.

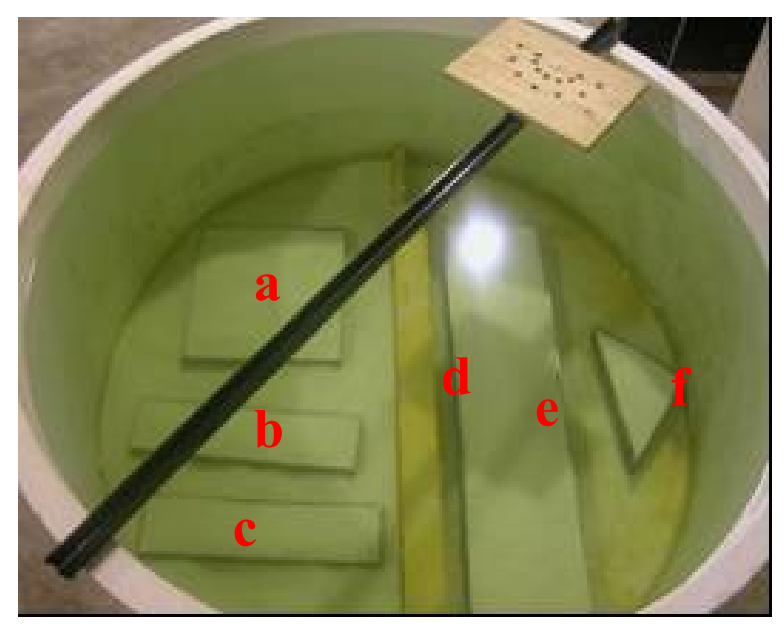

Figure 43: Top view of the tank with diagonal I-beam. Plastic beads in and around 3 metal forms (left) and in $\mathbf{2}$ metal forms (right). Sonar suspended from the U-channel. 


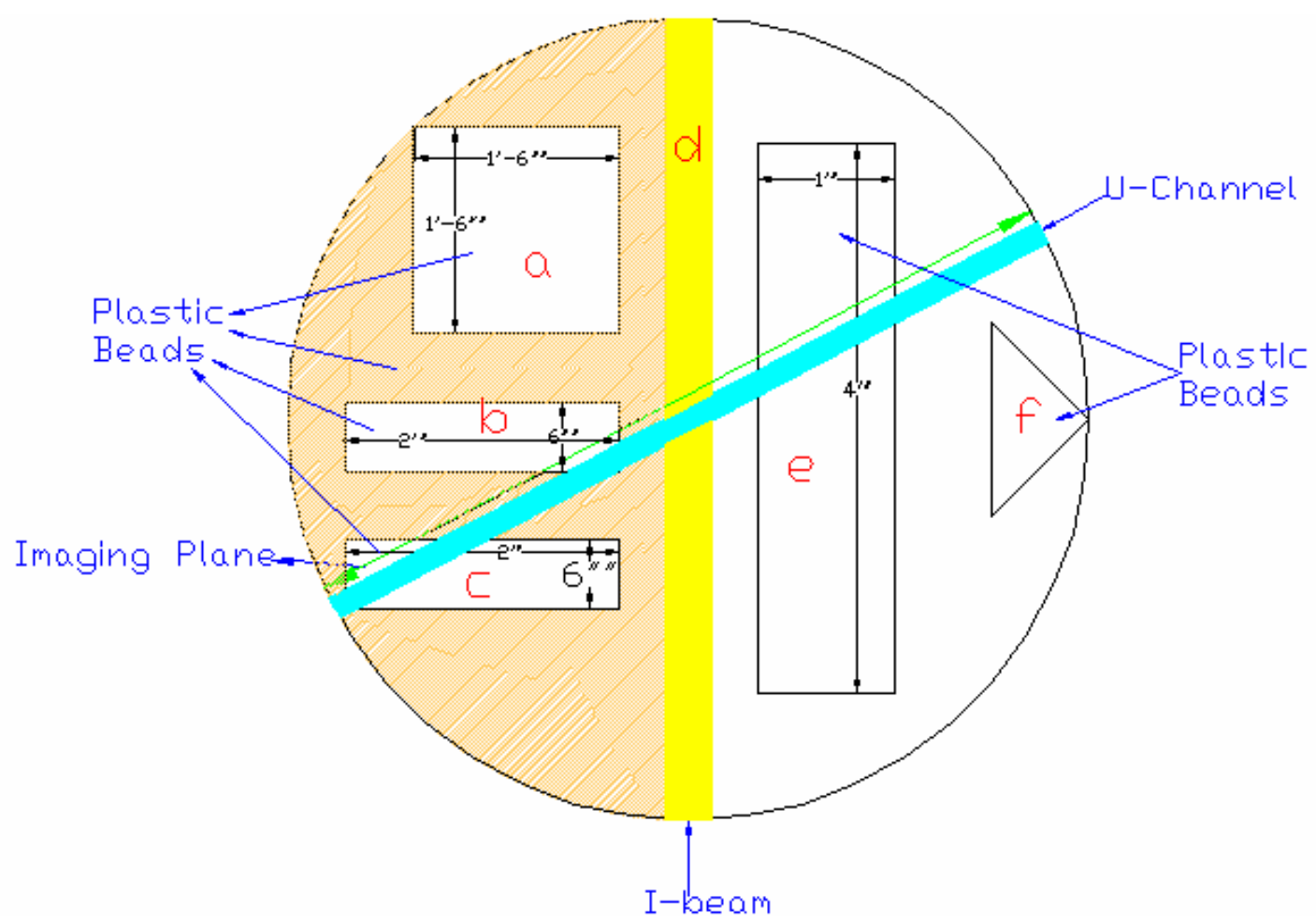

Figure 44: Top view of the tank showing the placement of the geometric forms ("a", "b", "c", "d", "e" and " $f$ ) and image plane of the sonar.

Figure 45 shows the image to be generated by sonar when sonar is placed at center of tank and at an angle $62^{\circ}$ as shown in Figure 44.

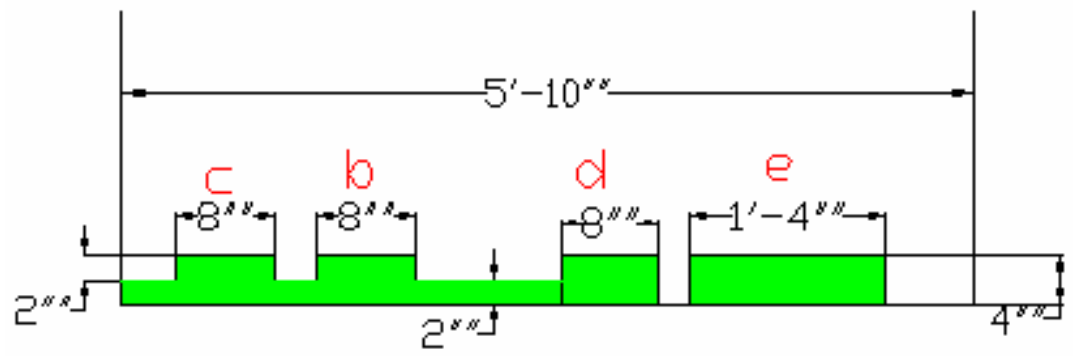

Figure 45: Side view of the sonar scan plane.

\subsubsection{Results}

Figure 46 is the 2-D image generated by sonar when it is scanning the plane shown in Figures 39 and 40. 


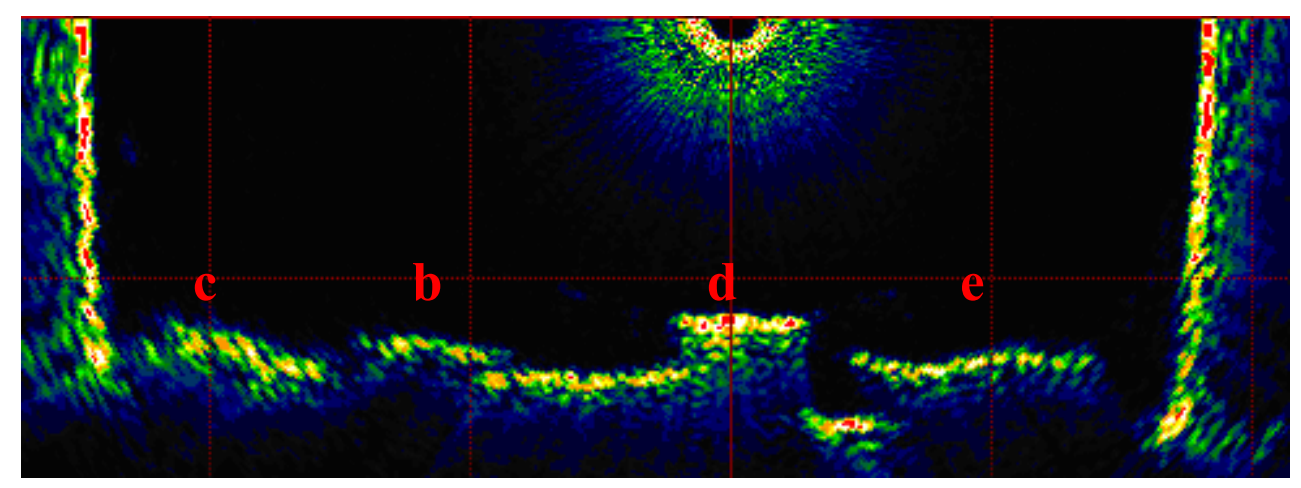

Figure 46: Image generated when plastic beads are placed inside the tank. "b", "c", "d" and "e" are the objects as shown in Figures 43 and 44.

In Figure 46 the $1^{\text {st }}$ hump $\left(\mathrm{c}^{*}\right)$ represents beads filling an 18-in. $\mathrm{x} 18$-in. metal form. The $2^{\text {nd }}$ hump $\left(b^{*}\right)$ corresponds to beads filling a 24-in. x 6-in. metal form. The tallest plateau is from the I-beam $\left(\mathrm{d}^{*}\right)$ along the diagonal of the tank and the hump farthest to the right $\left(\mathrm{e}^{*}\right)$ are beads filling a 48-in. x 12-in. metal form. From the image (Figure 44) it is evident that there are no beads present at the right of "d", however 2 in. of beads are present to the left of "d".

\subsubsection{Analyses}

The actual and the observed (by sonar software) measurements, in addition, to the relative error for the metal object are shown in Table 7. Precise tape measurements were taken for the actual distances and shown to be accurate within 1\%. Sound speed for determining the measurements of the objects by sonar was $1486 \mathrm{~m} / \mathrm{s}$ or $4875.3 \mathrm{ft} / \mathrm{s}$.

\footnotetext{
${ }^{*}$ Ref to Figure 43 and 44
} 
Table 7: Actual and Observed Locations and Dimensions of Different Metal Forms Inside a Tank.

\begin{tabular}{|l|l|l|l|l|l|l|}
\hline \multirow{2}{*}{ Position of sonar } & \multicolumn{3}{|c|}{$\mathrm{c}$ (Height) } & \multicolumn{3}{c|}{$\mathrm{b}$ (Height) } \\
\cline { 2 - 7 } & $\begin{array}{l}\text { Actual } \\
(\mathrm{ft})\end{array}$ & $\begin{array}{l}\text { Observed } \\
(\mathrm{ft})\end{array}$ & $\begin{array}{l}\text { Relative } \\
\text { Error }\end{array}$ & $\begin{array}{l}\text { Actual } \\
(\mathrm{ft})\end{array}$ & $\begin{array}{l}\text { Observed } \\
(\mathrm{ft})\end{array}$ & $\begin{array}{l}\text { Relative } \\
\text { Error }\end{array}$ \\
\hline Center of tank & 0.167 & 0.166 & $0.6 \%$ & 0.167 & 0.166 & $0.6 \%$ \\
\hline Near to tank wall & 0.167 & 0.166 & $0.6 \%$ & 0.167 & 0.167 & $0.0 \%$ \\
\hline & \multicolumn{3}{|c|}{$\mathrm{e}$ (Height) } & \multicolumn{3}{c|}{$\mathrm{e}$ (width) } \\
\hline Center of tank & 0.333 & 0.332 & $0.3 \%$ & 1.300 & 1.304 & $-0.3 \%$ \\
\hline Near to tank wall & 0.333 & 0.331 & $0.6 \%$ & 1.300 & 1.306 & $-0.4 \%$ \\
\hline & \multicolumn{3}{|c|}{$\mathrm{d}$ (Height on right side) } & \multicolumn{2}{c|}{$\mathrm{d}$ (Height on left side) } \\
\hline Center of tank & 0.500 & 0.499 & $0.0 \%$ & 0.333 & 0.331 & $0.6 \%$ \\
\hline Near to tank wall & 0.500 & 0.499 & $0.0 \%$ & 0.333 & 0.331 & $0.6 \%$ \\
\hline & \multicolumn{3}{|c|}{$\mathrm{d}$ (Width (I-beam) } & Sonar height from tank bottom \\
\hline Center of tank & 0.666 & 0.670 & $-0.6 \%$ & 2.200 & 2.198 & $0.4 \%$ \\
\hline Near to tank wall & 0.666 & 0.669 & $-0.4 \%$ & 2.200 & 2.188 & $0.5 \%$ \\
\hline
\end{tabular}

In Table 7 the observed value for the width of the objects is more than the actual values, because Imagenex $881 \mathrm{~A}$ profiling sonar sends out number of shots of acoustic wave in form of conical beam (having angle $1.4^{\circ}$ ) at an angle of $0.3^{\circ}$ each. This conical beam expands in diameter after traveling certain distance. When this expanded wave strikes the edge of any object in such a way that half of it fall on the object and other half doesn't, it creates a problem for sonar transducer to plot a point at that position. When such wave is detected by the sonar transducer, it plots a point considering that entire wave is reflected by the object leading to incorrect dimensions in the width of the objects. This also causes the smoothing of the object shape rather than having sharp edges. 


\subsection{Experiment 5}

\subsubsection{Objective}

To demonstrate the ability of sonar to accurately measure the objects in near proximity to the sonar head. The objective was to find the threshold distance above which the sonar is able to detect objects with accurate measurements.

\subsubsection{Setup}

This experiment was again performed in a fiberglass tank (70 in. diameter $\mathrm{x} 33$ in. height) with six bricks placed at the bottom as shown in Figure 47. This experiment was conducted in two parts. In the first part to find the minimal distance sonar was suspended at the center of the tank. A metallic plate (2 ft. height $\times 2 \mathrm{ft}$. width) acting as a shield was placed at four different distances (10 in., 13 in., 14 in., and 16 in.) from the sonar head. In second part sonar was placed at six different heights $(2 \mathrm{ft} ., 1.5 \mathrm{ft} ., 1 \mathrm{ft} ., 0.8 \mathrm{ft} ., 0.6 \mathrm{ft} .$, and $0.5 \mathrm{ft}$.) from the bottom of tank. The experiment was based on the following observations

a) Degradation of the signal strength of the sonar;

b) Differences of distances measured by sonar software to actual measurements of bricks.

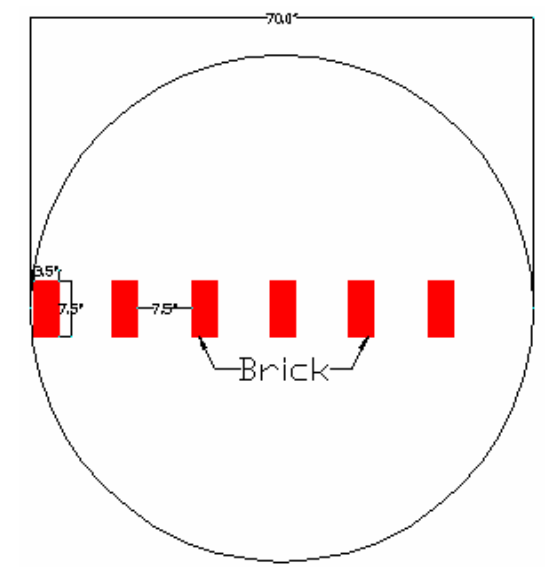

Figure 47: Top view of the tank with bricks placed inside it. 


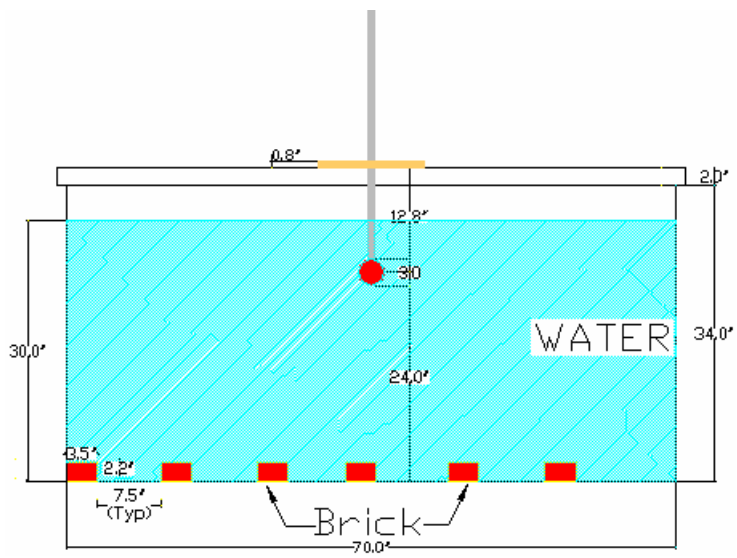

Figure 48: Cross section of the tank with the placement of the bricks and the sonar.

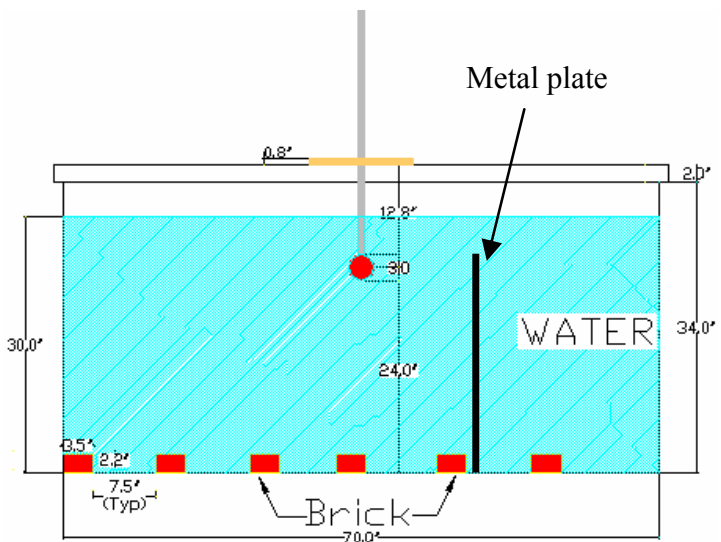

Figure 49: Cross section of the tank with metal plate placed in it at $\mathbf{1 . 5} \mathbf{f t}$. from sonar head.

Figure 48 shows the placement of the sonar and bricks. In this case the sonar was lowered from $2 \mathrm{ft}$. to $0.5 \mathrm{ft}$. In Figure 49 a metal place is placed next to a brick its $2 \mathrm{ft}$. from the sonar head. This metal plate was moved towards the sonar head from 16 in. to $10 \mathrm{in}$.

\subsubsection{Effect of a vertical metal plate placed near the sonar head}

Following images (Figures 50, 51, 52, and 53) were generated by sonar software when metal plate was placed at four different distances from the sonar head. The contiguous points at the bottom of the each image show the brick.

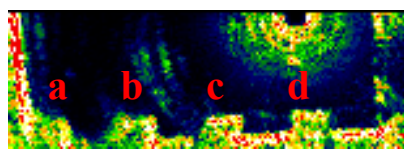

Figure 50: Image generated when metal plate is placed 10 in. from sonar.

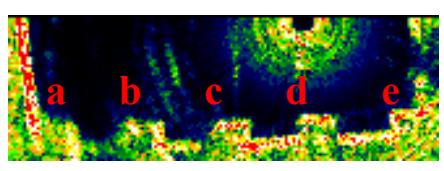

Figure 51: Image generated when metal plate is placed 13 in. from sonar.

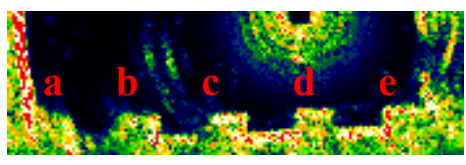

Figure 52: Image generated when metal plate is placed $14 \mathrm{in.}$ from sonar.

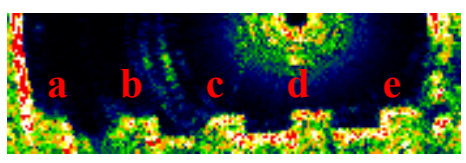

Figure 53: Image generated when metal plate is placed 16 in. from sonar. 
Analyses

Actual and the observed measurements, in addition to the relative error for each brick are shown in Tables 8 and 9. Precise tape measurements were taken for the actual distances were and shown to be accurate within $1 \%$. Sound speed for determining the measurements of the objects by sonar was $1486 \mathrm{~m} / \mathrm{s}$ or $4875.3 \mathrm{ft} / \mathrm{s}$.

Table 8: Actual and Observed Measurements (with Relative Error) of Metal Plate.

\begin{tabular}{|c|c|c|}
\hline $\begin{array}{c}\text { Distance of metal plate } \\
\text { from the sonar head (ft) }\end{array}$ & $\begin{array}{c}\text { Distance measure by sonar } \\
\text { software (ft) }\end{array}$ & Relative Error \\
\hline 1.33 & 1.330 & $0.0 \%$ \\
\hline 1.16 & 1.158 & $0.1 \%$ \\
\hline 1.08 & 1.080 & $0.0 \%$ \\
\hline 0.83 & 0.831 & $-0.1 \%$ \\
\hline
\end{tabular}

Table 9: Actual and Observed Measurements (with Relative Error) of Distances between the Bricks.

\begin{tabular}{|c|c|c|c|c|c|c|}
\hline \multirow{2}{*}{$\begin{array}{l}\text { Distance of the } \\
\text { metal plate from } \\
\text { the sonar (ft) }\end{array}$} & \multicolumn{3}{|c|}{ Distance between a and $\mathrm{b}$} & \multicolumn{3}{|c|}{ Distance between b and c } \\
\cline { 2 - 7 } & Actual & $\begin{array}{c}\text { Observed } \\
\text { (software) } \\
(\mathrm{ft})\end{array}$ & $\begin{array}{c}\text { Relative } \\
\text { Error }\end{array}$ & $\begin{array}{c}\text { Actual } \\
(\mathrm{ft})\end{array}$ & $\begin{array}{c}\text { Observed } \\
\text { (software) } \\
(\mathrm{ft})\end{array}$ & $\begin{array}{c}\text { Relative } \\
\text { Error }\end{array}$ \\
\hline 1.33 & 0.62 & 0.622 & $-0.3 \%$ & 0.62 & 0.624 & $-0.3 \%$ \\
\hline 1.16 & 0.62 & 0.622 & $-0.3 \%$ & 0.62 & 0.622 & $-0.3 \%$ \\
\hline 1.08 & 0.62 & 0.622 & $-0.3 \%$ & 0.62 & 0.622 & $-0.3 \%$ \\
\hline 0.83 & 0.62 & 0.622 & $-0.3 \%$ & 0.62 & 0.625 & $-0.4 \%$ \\
\hline & Distance between c and d & Distance between d and e \\
\hline 1.33 & 0.62 & 0.623 & $-0.3 \%$ & 0.62 & 0.622 & $-0.3 \%$ \\
\hline 1.16 & 0.62 & 0.625 & $-0.4 \%$ & 0.62 & 0.622 & $-0.3 \%$ \\
\hline 1.08 & 0.62 & 0.625 & $-0.4 \%$ & 0.62 & 0.622 & $-0.3 \%$ \\
\hline 0.83 & 0.62 & 0.625 & $-0.4 \%$ & - & - & - \\
\hline
\end{tabular}




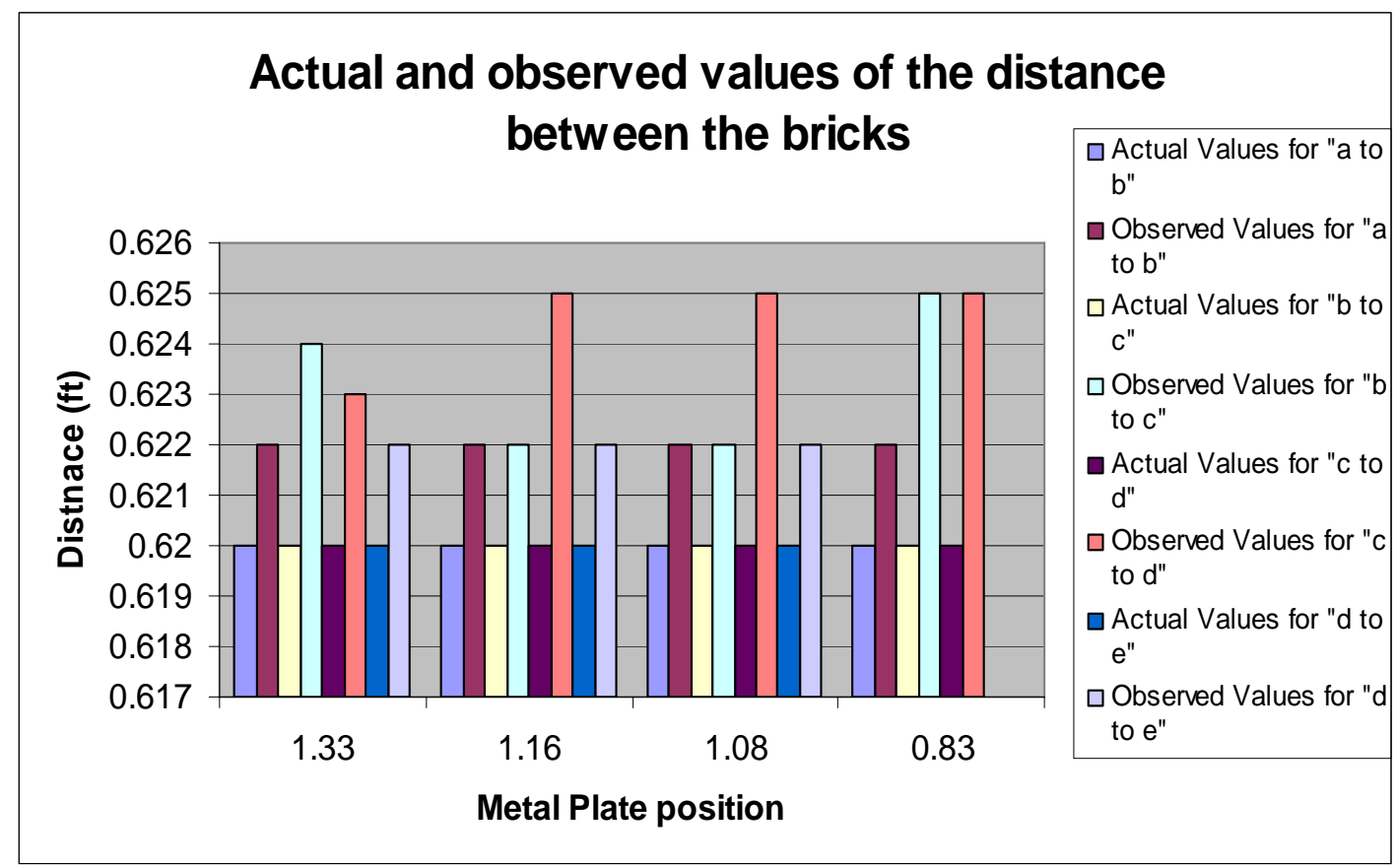

Figure 54: Plot of relative errors in measurements of distance between bricks when metal plate is placed at different positions.

From the bar plot of relative errors in Figure 54 And the values in Tables 8 and 9 it can be concluded that sonar can detect the objects accurately when it is $10 \mathrm{in}$. away from the objects. Hence the minimum distance sonar can be placed from the tank wall is $0.83 \mathrm{ft}$. or 10 in.

\subsubsection{Effect of sonar placed near a horizontal solids layer}

Following images were obtained from sonar software when sonar head was placed at six different heights form the tank bottom. The contiguous points at tank bottom represent the bricks placed inside the tank.

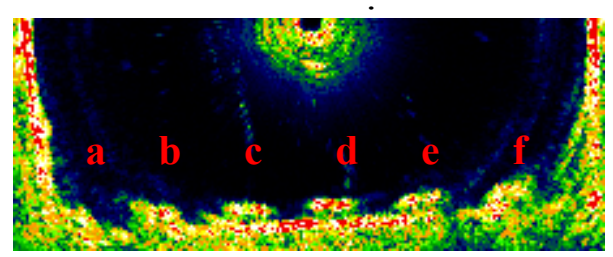

Figure 55: Image generated when sonar head is suspended $2 \mathrm{ft}$. from tank bottom.

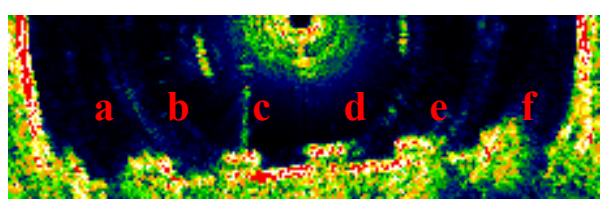

Figure 56: Image generated when sonar head is suspended $1.5 \mathrm{ft}$. from tank bottom. 


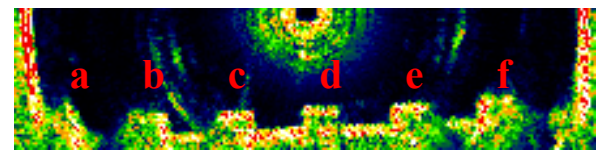

Figure 57: Image generated when sonar head is suspended $1 \mathrm{ft}$. from tank bottom.

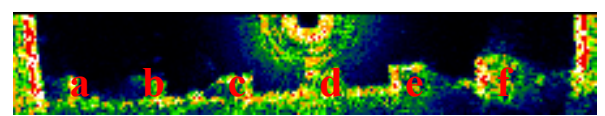

Figure 58: Image generated when sonar head is suspended $0.8 \mathrm{ft}$. from tank bottom.

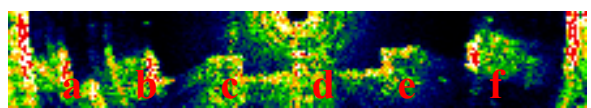

Figure 59: Image generated when sonar head is suspended $0.6 \mathrm{ft}$. from tank bottom.

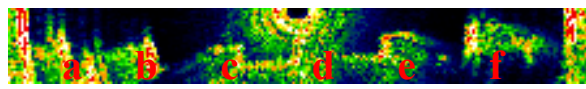

Figure 60: Image generated when sonar head is suspended $0.5 \mathrm{ft}$. from tank bottom

Analyses

Height and the width of bricks were calculated at each distance. Actual and the observed measurements, in addition to the relative error for each brick, are shown in Tables 10, 11, and 12. Precise tape measurements were taken for the actual distances were and shown to be accurate within 1\%. Sound speed for determining the measurements of the objects by sonar was $1486 \mathrm{~m} / \mathrm{s}$ or $4875.3 \mathrm{ft} / \mathrm{s}$.

Table 10: Actual and Observed Measurements (with Relative Error) of Tank.

\begin{tabular}{|c|c|c|c|c|c|}
\hline \multicolumn{2}{|c|}{ Distance of sonar form bottom of tank } & \multicolumn{3}{|c|}{ Diameter of the tank } \\
\hline $\begin{array}{c}\text { Actual } \\
(\mathrm{ft})\end{array}$ & $\begin{array}{c}\text { Observed } \\
(\text { software }) \\
(\mathrm{ft})\end{array}$ & $\begin{array}{c}\text { Relative } \\
\text { Error }\end{array}$ & $\begin{array}{c}\text { Actual } \\
(\mathrm{ft})\end{array}$ & $\begin{array}{c}\text { Observed } \\
(\text { software }) \\
(\mathrm{ft})\end{array}$ & $\begin{array}{c}\text { Relative } \\
\text { Error }\end{array}$ \\
\hline 2.00 & 2.000 & $0.0 \%$ & 5.70 & 5.696 & $0.0 \%$ \\
\hline 1.50 & 1.496 & $0.2 \%$ & 5.70 & 5.689 & $0.2 \%$ \\
\hline 1.00 & 0.999 & $0.1 \%$ & 5.70 & 5.699 & $0.1 \%$ \\
\hline 0.80 & 0.798 & $0.2 \%$ & 5.70 & 5.696 & $0.1 \%$ \\
\hline 0.60 & 0.600 & $0.0 \%$ & 5.70 & 5.699 & $0.1 \%$ \\
\hline 0.50 & 0.510 & $-2.0 \%$ & 5.70 & 5.551 & $2.6 \%$ \\
\hline
\end{tabular}


Table 11: Actual and Observed Measurements (with Relative Error) of Distance between the Bricks.

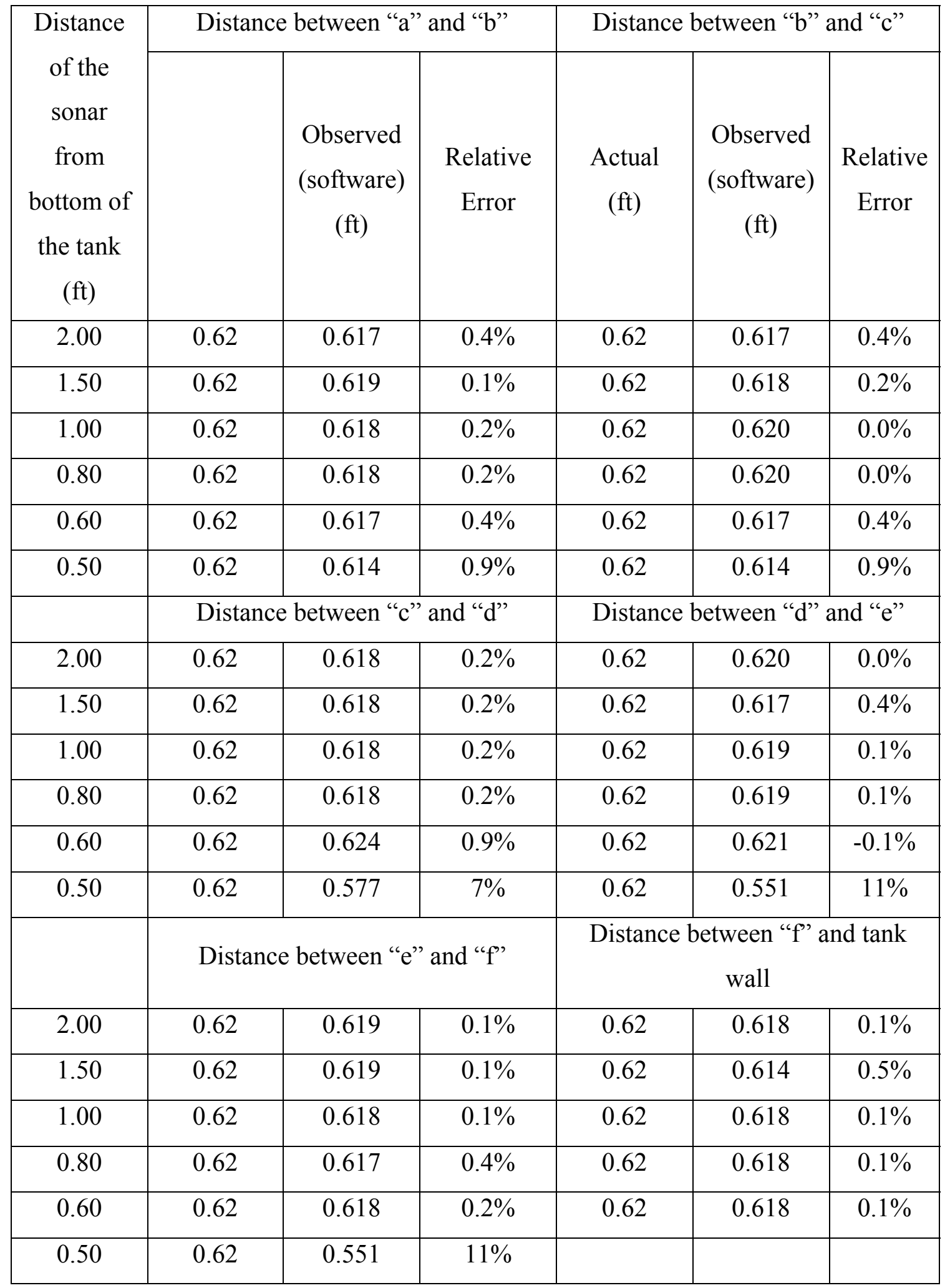




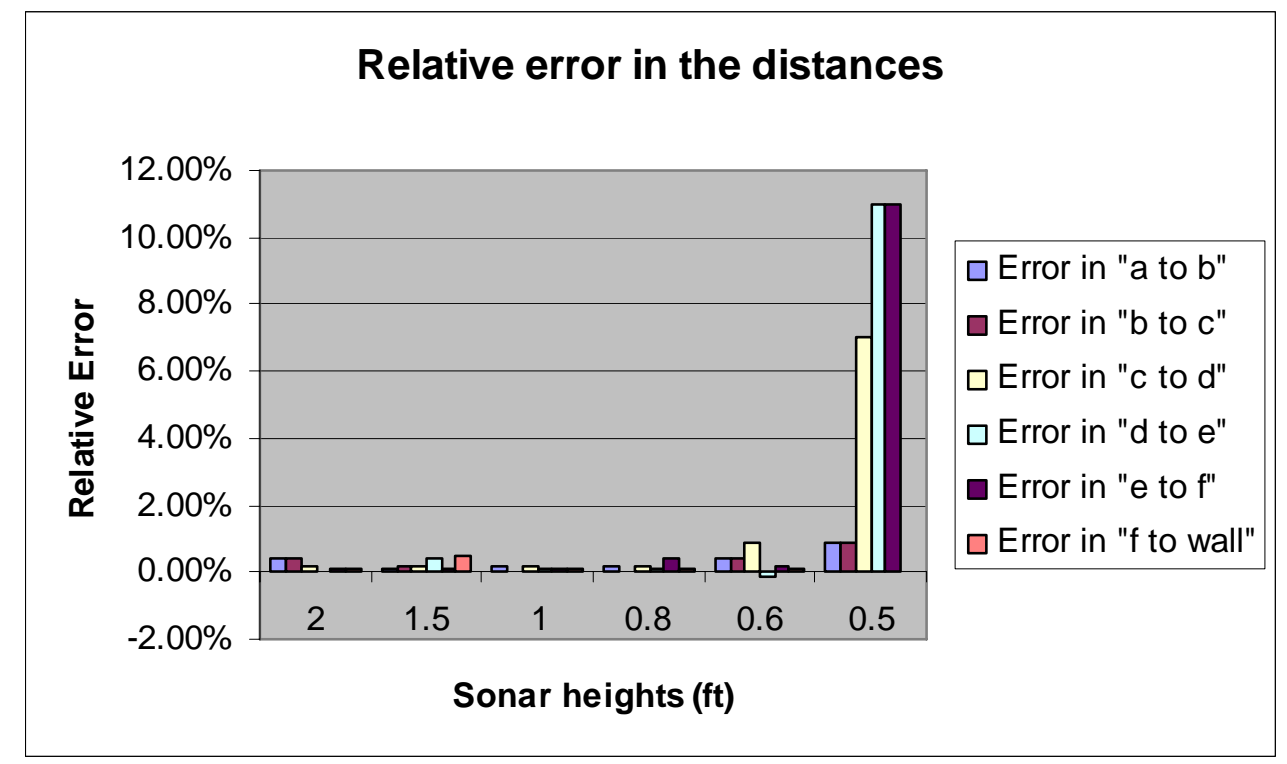

Figure 61: Relative error in the measurements of the distance between the bricks when sonar is placed at different heights from tank bottom.

Form the plot of the Figure 60, it can is clear that minimum distance sonar can placed to the tank bottom is $0.6 \mathrm{ft}$. If the distance is decrease then the relative error is increased this is because the image looses its resolution after this distance and it becomes difficult to distinguish one object from another.

To verify this result, relative error between the heights of the bricks when sonar was placed at different heights was calculated. Table 12 summarizes the actual and the observed and the relative errors in the heights of the bricks when sonar is placed at different heights. The actual measurements taken have $1 \%$ accuracy. 
Table 12: Actual and Observed Measurements (with Relative Error) of Brick Heights.

\begin{tabular}{|c|c|c|c|c|c|c|}
\hline Distance & \multicolumn{3}{|c|}{ Height of the brick "a" } & \multicolumn{3}{|c|}{ Height of the brick "b" } \\
\hline $\begin{array}{c}\text { sonar } \\
\text { from } \\
\text { bottom of } \\
\text { the tank } \\
(\mathrm{ft})\end{array}$ & $\begin{array}{c}\text { Actual } \\
(\mathrm{ft})\end{array}$ & $\begin{array}{c}\text { Observed } \\
\text { (software) } \\
(\mathrm{ft})\end{array}$ & $\begin{array}{c}\text { Relative } \\
\text { Error }\end{array}$ & $\begin{array}{c}\text { Actual } \\
(\mathrm{ft})\end{array}$ & $\begin{array}{c}\text { Observed } \\
\text { (software) } \\
\text { (ft) }\end{array}$ & $\begin{array}{c}\text { Relative } \\
\text { Error }\end{array}$ \\
\hline 1.50 & 0.18 & 0.179 & $0.5 \%$ & - & - & - \\
\hline 1.00 & 0.18 & 0.180 & $0.0 \%$ & 0.18 & 0.180 & $0.0 \%$ \\
\hline 0.80 & 0.18 & 0.180 & $0.0 \%$ & 0.18 & 0.180 & $0.0 \%$ \\
\hline 0.60 & 0.18 & 0.180 & $0.0 \%$ & 0.18 & 0.180 & $0.0 \%$ \\
\hline & \multicolumn{3}{|c|}{ Height of the brick "c" } & \multicolumn{3}{|c|}{ Height of the brick "d" } \\
\hline 1.50 & 0.18 & 0.180 & $0.0 \%$ & 0.18 & 0.180 & $0.0 \%$ \\
\hline 1.00 & 0.18 & 0.180 & $0.0 \%$ & 0.18 & 0.180 & $0.0 \%$ \\
\hline 0.80 & 0.18 & 0.180 & $0.0 \%$ & 0.18 & 0.180 & $0.0 \%$ \\
\hline 0.60 & 0.18 & 0.180 & $0.0 \%$ & 0.18 & 0.180 & $0.0 \%$ \\
\hline \multirow[t]{2}{*}{0.50} & 0.18 & 0.184 & $-2.2 \%$ & & & \\
\hline & \multicolumn{3}{|c|}{ Height of the brick "e" } & \multicolumn{3}{|c|}{ Height of the brick " $\mathrm{f}$ " } \\
\hline 1.50 & 0.18 & 0.180 & $2.2 \%$ & 0.18 & 0.180 & $0.0 \%$ \\
\hline 1.00 & 0.18 & 0.179 & $0.5 \%$ & 0.18 & 0.179 & $0.5 \%$ \\
\hline 0.80 & 0.18 & 0.179 & $0.5 \%$ & 0.18 & 0.180 & $0.0 \%$ \\
\hline 0.60 & 0.18 & 0.180 & $0.0 \%$ & 0.18 & 0.180 & $0.0 \%$ \\
\hline 0.50 & 0.18 & 0.184 & $-2.2 \%$ & $\begin{array}{ll}- & \text { r }\end{array}$ & - & - \\
\hline
\end{tabular}

Form Table 12 it is verified that minimum distance sonar to be placed to tank bottom is $0.6 \mathrm{ft}$. At $0.5 \mathrm{ft}$ the error increases as the reflection form the tank bottom increases resulting in distorted images. The mage looses its resolution at this distance. 


\subsection{Experiment 6}

\subsubsection{Objective}

To analyze the chemical compatibility of the sonar and its cable to one year of extended exposure to HLW tank. The purpose of this experiment is to demonstrate that the sonar head and cable can withstand the expected extended exposure to a highly caustic solution $(\mathrm{pH}>14)$ to determine the lifetime of the sonar in the HLW tank.

\subsubsection{Setup}

The sonar along with its cable was immersed in a stainless steel container (14 in. diameter x 27 in. height) containing 16 gal. of caustic solution (see Figure 62 below). The solution was of similar chemical content as Hanford wastes and with similar alkalinity $\mathrm{pH}>14$. This solution was heated to $45^{\circ} \mathrm{C}$, the temperature $10^{\circ} \mathrm{C}$ warmer than the highest expected in the tank of high-level radioactive waste to facilitate accelerated any chemical degradation. The sonar with cable attached were kept in the heated caustic solution for a period of 24 hours and then removed and rinsed. The surfaces of the sonar, cables, and cable connector were observed under magnification to ascertain if there is appreciable chemical degradation anywhere. If, no appreciable degradation was observed, sonar was to be placed back into the heated solution for 48 hours. The same observations were done after 48 and 72 hour exposures. At the end of all three exposures the sonar was tested by placing it in another stainless drum (23 $\frac{1}{2}$ in. diameter and 34 in. high) to image bricks placed in water. Two bricks were placed on top of each other (to give a height of 4.4 in.) at the bottom of the drum such that the sonar beam cuts then through center (Figure 64). 


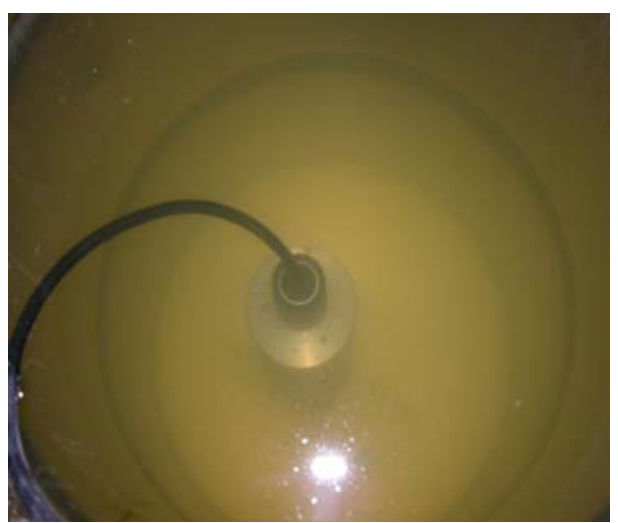

Figure 62: Top view of the drum with sonar placed in caustic solution.
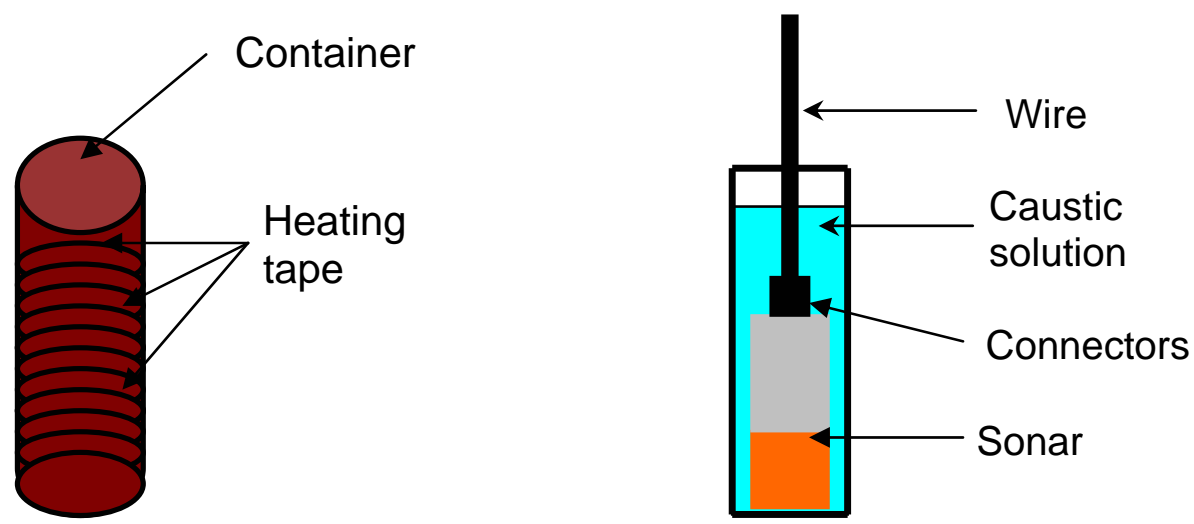

Figure 63: The drum with tape heater wound around it (left) and the placement of sonar inside the drum (right).

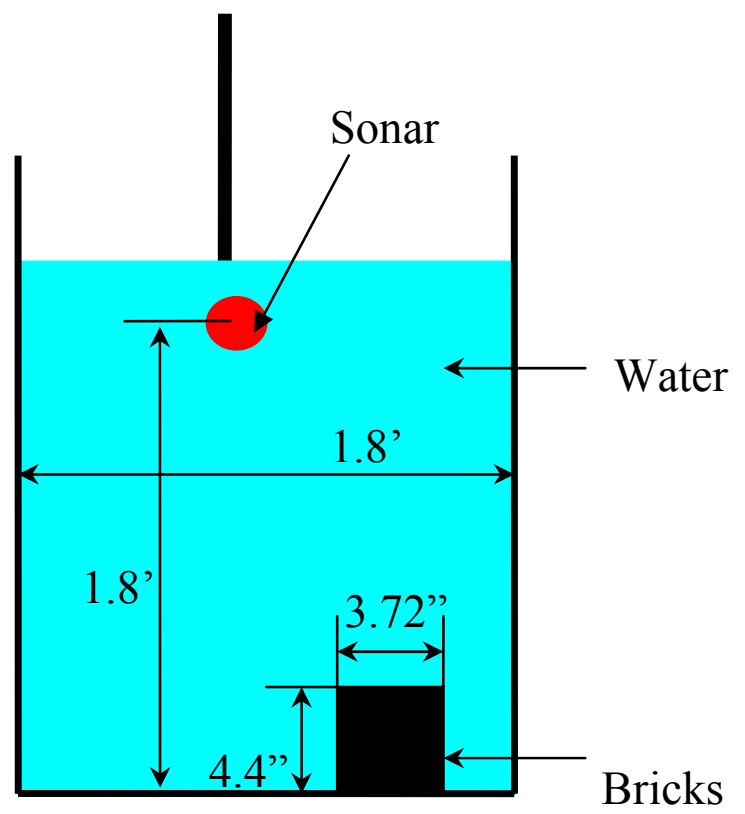

Figure 64: Cross-section of the steel drum, with bricks and sonar. 


\subsubsection{Results}

After keeping the sonar for 24 hours in caustic solution at $45^{\circ} \mathrm{C}$, it was found that an aluminum band around the Polyurethane was destroyed. It was eaten away by the caustic solution. Figure 65 shows the image of the aluminum band around the sonar.

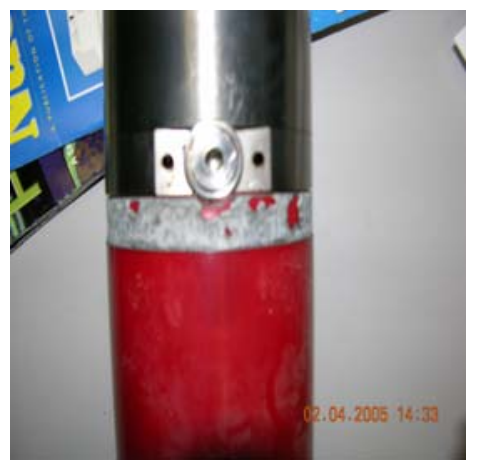

Figure 65: Aluminum band destroyed by caustic solution.

This band was removed from the sonar head and replaced by a 316-stainless band. The sonar was tested for its accuracy again in steel drum show in Figure 66.

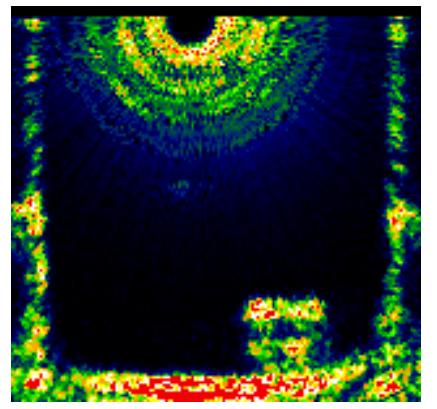

Figure 66: Image obtained by sonar for the arrangement shown in Figure 64.

\subsubsection{Analysis}

Actual, observed, and relative error between the heights was calculated and is shown in Table 13. Precise tape measurements were taken for the actual distances were and shown to be accurate within 1\%. Sound speed for determining the measurements of the objects by sonar was $1486 \mathrm{~m} / \mathrm{s}$ or $4875.3 \mathrm{ft} / \mathrm{s}$. 
Table 13: Actual and Observed Dimensions of the Objects with its Relative Error.

\begin{tabular}{|c|c|c|c|}
\hline & Actual (ft) & Observed (ft) & Relative Error \\
\hline Height of the sonar & 1.8 & 1.811 & $0.06 \%$ \\
\hline Height of the bricks & 0.37 & 0.367 & $0.08 \%$ \\
\hline Width of the bricks & 0.31 & 0.312 & $0.06 \%$ \\
\hline
\end{tabular}

Sonar was again tested after keeping it in caustic solution for 48 and 72 hour at $45^{\circ} \mathrm{C}$.

Results obtained were the same as that of Table 13 (less than $0.5 \%$ variation in relative error).

\subsection{Experiment 7}

\subsubsection{Objective}

To examine the performance of sonar in caustic solution. Data obtained from sonar was used to calibrate the sonar measurements by calculating the correct sound speed for each density and temperature.

\subsubsection{Setup}

The setup consisted of a 55 gal stainless steel drum placed on top of a heater which itself was positioned on the spill containment (Figure 67). A stand made of Uchannel was placed at the bottom of the drum. This U-channel stand was placed to avoid precipitation of salt that could affect the image accuracy. Two stainless steel objects 3 in. $x 2$ in. $x 3$ in. and 6 in. $x 6$ in. $x 6$ in. were welded 4 in. apart on this stand. Drum was filled with 40 gal of tap water having density $1 \mathrm{~g} / \mathrm{cm}^{3}$. Sonar was be suspended at 24 in. from drum bottom with the help of U-channel. U- Channel was fixed at the drum top and sonar was held at two places to make it horizontal to the drum bottom. The density of the water was changed from 1 to $1.4 \mathrm{~g} / \mathrm{cm}^{3}$ with increments of $0.1 \mathrm{~g} / \mathrm{cm}^{3}$. This was done by 
adding sodium nitrate into the water. Also the temperature of solution was changed from $25^{\circ} \mathrm{C}$ to $35^{\circ} \mathrm{C}$ with $5^{\circ}$ increments.

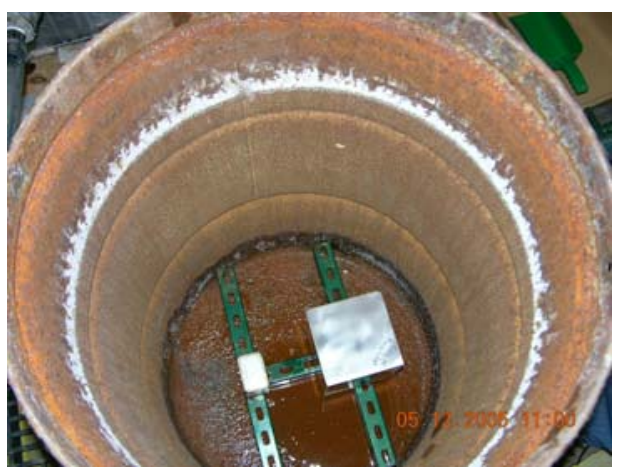

Figure 67: Top view of the drum with two stainless steel objects placed on U-channel stand.

A thermo-couple with a controller was added to this setup to maintain the required temperature. A portable $\mathrm{pH}$ meter was be used to measure the $\mathrm{pH}$ of the solution at each density.

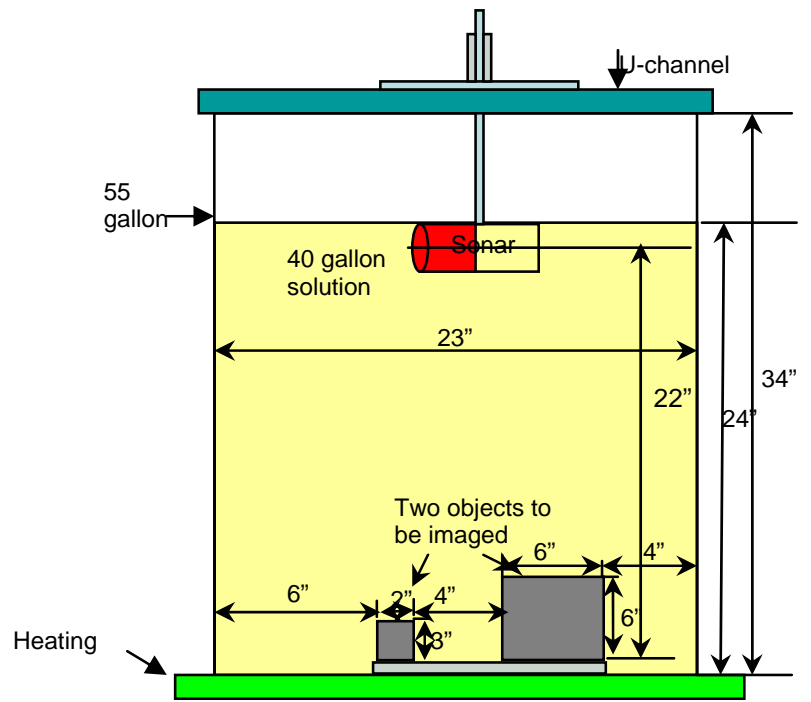

Figure 68: Cross-section of the steel drum, with metal pieces and sonar.

Sodium nitrate solution was prepared in different drum of 60 gal capacity. A mixer was attached to the wall of this drum to stir the solution while sodium nitrate was added to the solution (Figure 69). 


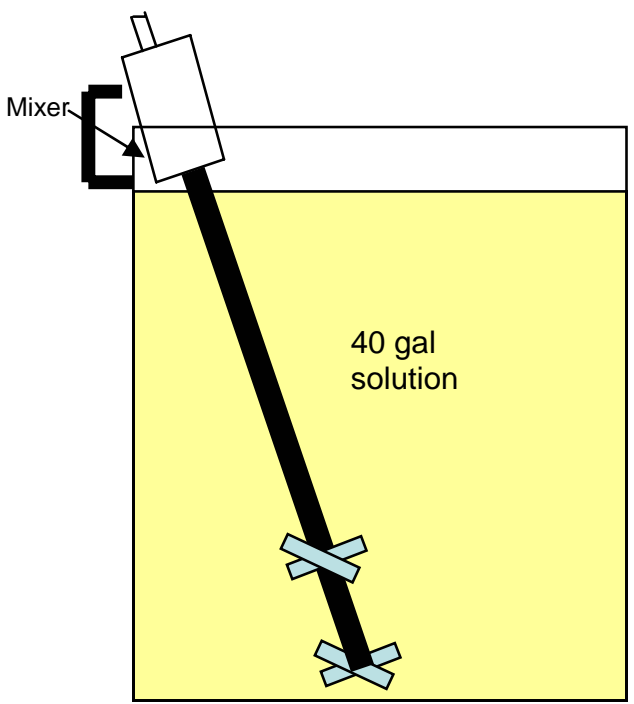

Figure 69: Placement of mixer in the drum filled with sodium nitrate solution.

\subsubsection{Results}

The results were recorded at constant sound speed i.e., $1500 \mathrm{~m} / \mathrm{s}$ or $4921.3 \mathrm{ft} / \mathrm{s}$. The range was set at $3 \mathrm{ft}$ with gain of $40 \mathrm{~dB}$ (maximum gain sonar has). These settings were kept constant thought out the experiments. Only the density and the temperature of the solution were changed. Density was changed from $1 \mathrm{~g} / \mathrm{cm}^{3}$ to $1.4 \mathrm{~g} / \mathrm{cm}^{3}$ with increments of $0.1 \mathrm{~g} / \mathrm{cm}^{3}$ each time (i.e., $1 \mathrm{~g} / \mathrm{cm}^{3}, 1.1 \mathrm{~g} / \mathrm{cm}^{3}, 1.2 \mathrm{~g} / \mathrm{cm}^{3}, 1.3 \mathrm{~g} / \mathrm{cm}^{3}$, and 1.4 $\mathrm{g} / \mathrm{cm}^{3}$ ). For density of $1 \mathrm{~g} / \mathrm{cm}^{3}$ tap water was taken. Temperature was changed from $25^{\circ} \mathrm{C}$ to $35^{\circ} \mathrm{C}$ with $5^{\circ}$ increments (i.e., $25^{\circ} \mathrm{C}, 30^{\circ} \mathrm{C}$, and $35^{\circ} \mathrm{C}$ ).

When 40 gal of tap water was poured into the drum. Following images were obtained at three temperatures $25^{\circ} \mathrm{C}, 30^{\circ} \mathrm{C}$, and $35^{\circ} \mathrm{C}$. 

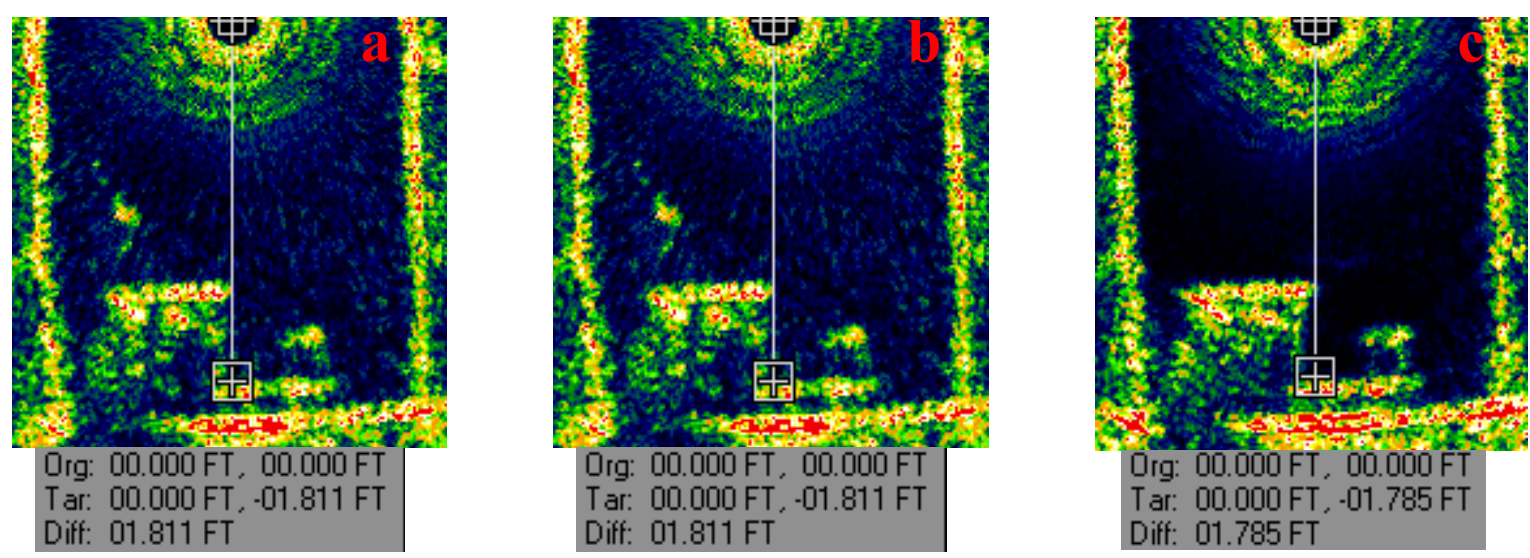

Figure 70: Images generated when water is temperature is heated to $25^{\circ} \mathrm{C}(\mathrm{a}), 30^{\circ} \mathrm{C}(\mathrm{b})$, and $35^{\circ} \mathrm{C}$ (c).

Water was replaced by sodium nitrate solution of $1.102 \mathrm{~g} / \mathrm{cm}^{3}$ (prepared by adding $50 \mathrm{lb}$ of sodium nitrate to 40 gal. of water). Following images were obtained at three temperatures at $25^{\circ} \mathrm{C}, 30^{\circ} \mathrm{C}$ and $35^{\circ} \mathrm{C}$. The $\mathrm{pH}$ of the solution was found to be 10.87 at $25^{\circ} \mathrm{C}$.
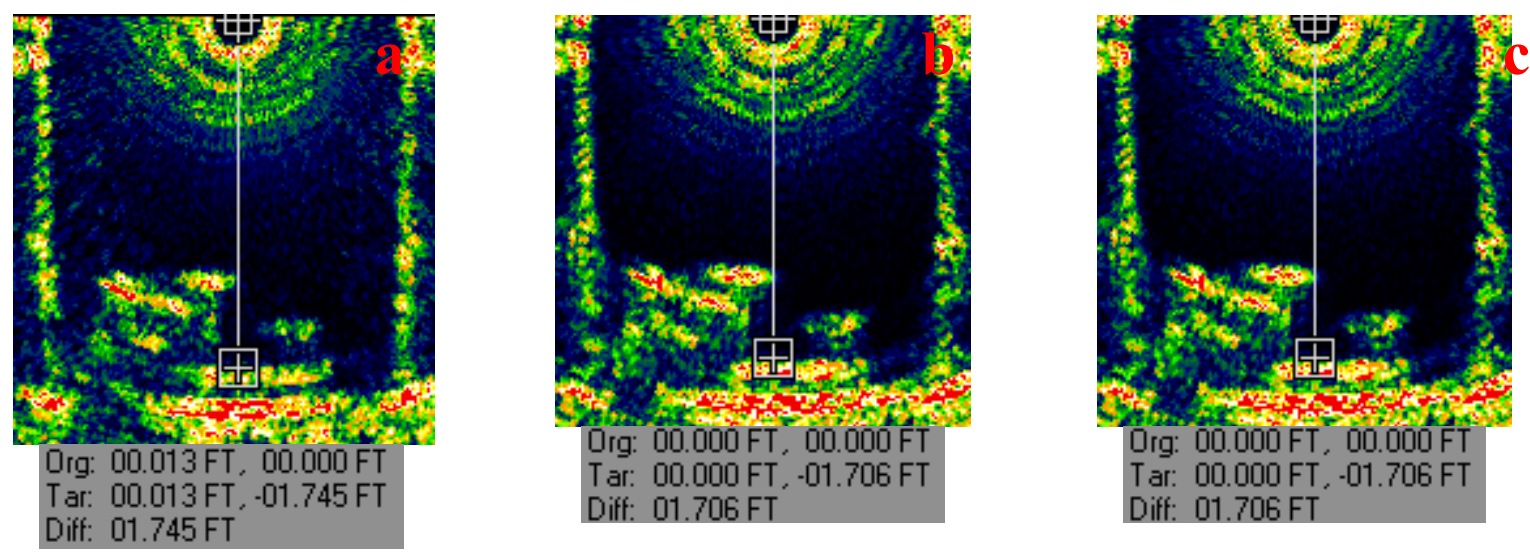
(c).

Figure 71: Image obtained when solution of $\rho=1.1 \mathrm{~g} / \mathrm{cm}^{3}$ is heated to $25^{\circ} \mathrm{C}(\mathrm{a}), 30^{\circ} \mathrm{C}(\mathrm{b})$, and $35^{\circ} \mathrm{C}$

Now the density of solution was further increased to $1.198 \mathrm{~g} / \mathrm{cm}^{3}$ by adding $85 \mathrm{lb}$ of more sodium nitrate to the already available sodium nitrate solution. The $\mathrm{pH}$ of the solution was measured at room temperature $\left(25^{\circ} \mathrm{C}\right)$ and was found to be 10.13 . Following images were obtained for three temperatures $\left(25^{\circ} \mathrm{C}, \quad 30^{\circ} \mathrm{C}\right.$, and $\left.35^{\circ} \mathrm{C}\right)$. 

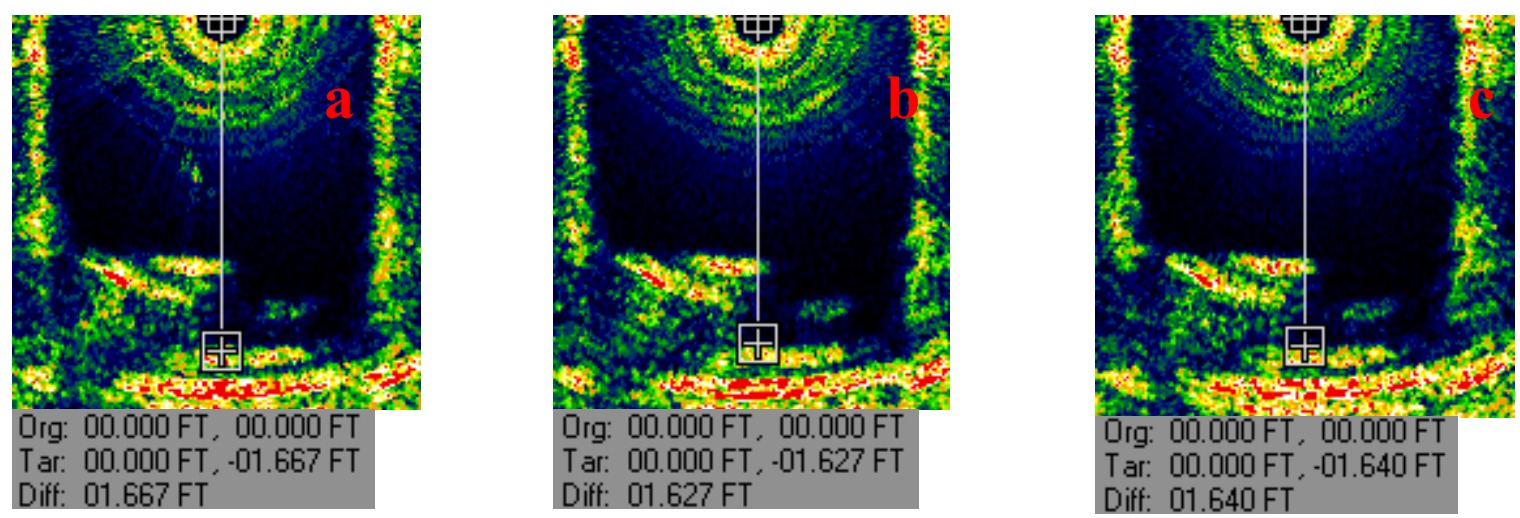

Figure 72: Image obtained when solution of $\rho=1.2 \mathrm{~g} / \mathrm{cm}^{3}$ is heated to $25^{\circ} \mathrm{C}(\mathrm{a}), 30^{\circ} \mathrm{C}(\mathrm{b})$, and $35^{\circ} \mathrm{C}$ (c).

Density of the solution was again increased to $1.282 \mathrm{~g} / \mathrm{cm}^{3}$ by adding $75 \mathrm{lb}$ of more sodium nitrate in the solution of $\rho=1.192 \mathrm{~g} / \mathrm{cm}^{3}$ (Prepared for earlier experiment). The $\mathrm{pH}$ of the solution was 9.97 at $25^{\circ} \mathrm{C}$. Following images were obtained for three temperatures $\left(25^{\circ} \mathrm{C}, 30^{\circ} \mathrm{C}\right.$, and $\left.35^{\circ} \mathrm{C}\right)$.
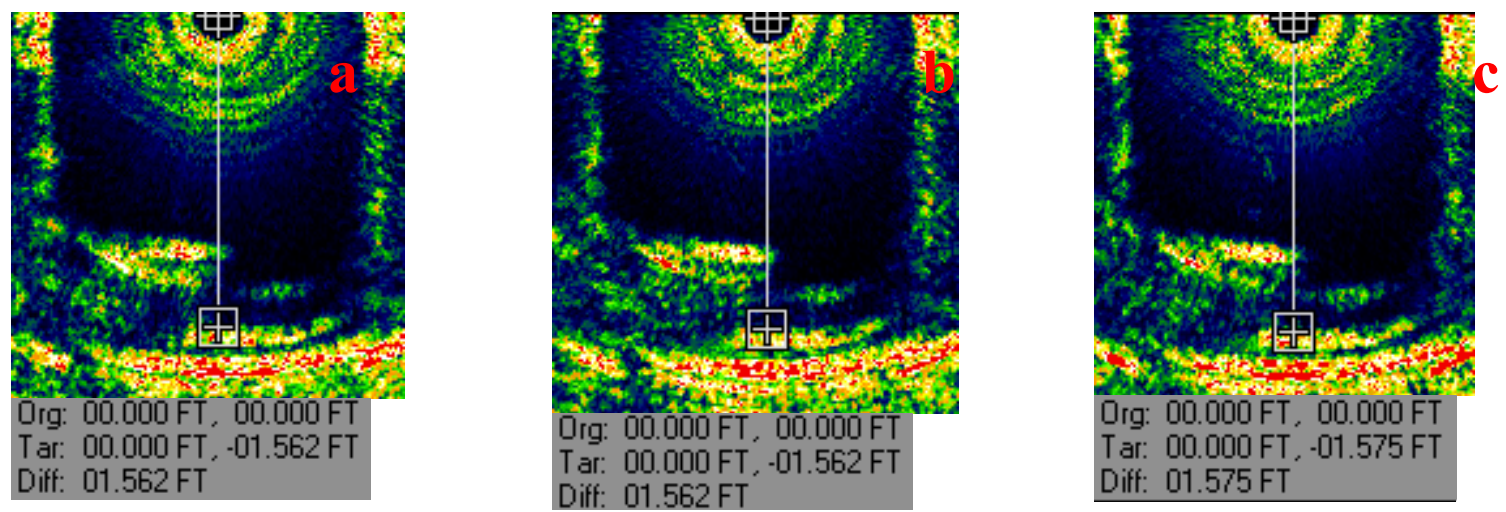

Figure 73: Image obtained when solution of $\rho=1.3 \mathrm{~g} / \mathrm{cm}^{3}$ is heated to $25^{\circ} \mathrm{C}(\mathrm{a}), 30^{\circ} \mathrm{C}(\mathrm{b})$, and $35^{\circ} \mathrm{C}$ (c).

Density of the solution was increased to $1.394 \mathrm{~g} / \mathrm{cm}^{3}$ by adding $105 \mathrm{lb}$ of more sodium nitrate to the solution of $\rho=1.282 \mathrm{~g} / \mathrm{cm}^{3}$ (prepared for earlier experiment). The $\mathrm{pH}$ of the solution was 9.72 at room temperature $\left(25^{\circ} \mathrm{C}\right)$. Following images were obtained at three different temperatures $\left(25^{\circ} \mathrm{C}, 30^{\circ} \mathrm{C}\right.$, and $\left.35^{\circ} \mathrm{C}\right)$. 

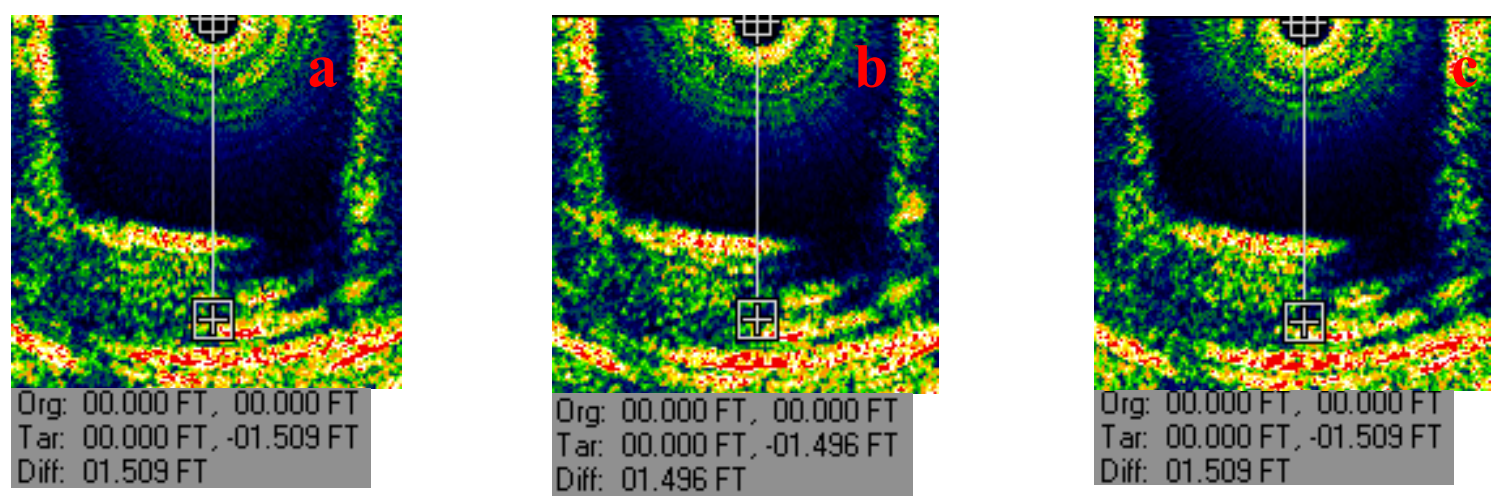

Figure 74: Image obtained when solution of $\rho=1.4 \mathrm{~g} / \mathrm{cm}^{3}$ is heated to $25^{\circ} \mathrm{C} \mathrm{(a),} 30^{\circ} \mathrm{C} \mathrm{(b),} \mathrm{and} 35^{\circ} \mathrm{C}$ (c).

\subsubsection{Analyses}

The measurements obtained from the images above were analyzed between three reference heights 1) height of the sonar from the U-Channel stand, 2) height of the cube, and 3) height of the rectangle. The errors in the heights were analyzed to find the correct the sound speed in the solution for specific temperature and density. The corrected sound speed was fed into the sonar software and the results were again analyzed by calculating the relative errors.

\section{Method to find the correct sound speed at particular temperature at specific density}

Sonar sends out a sound pulse from transducer and then precisely measure the time it takes for the sound pulses to be reflected back to the transducer. The distance to an object can be calculated using this time difference and the speed of sound in the medium.

$$
\text { Time }=\frac{\text { distance }}{\text { speed }}
$$

By using 4,1 formula the sound speed can be calculated if the distance between the any one object is know, which give rise to the following formula:

$$
\text { Correct sound speed }=\text { default sound } \text { speed } \frac{\text { Actual distance }}{\text { observed distance }}
$$


Software is written in Visual Basic 6 that takes the values form the sonar software and generates the correct sound speed in that medium. Code of software is in Appendix C.

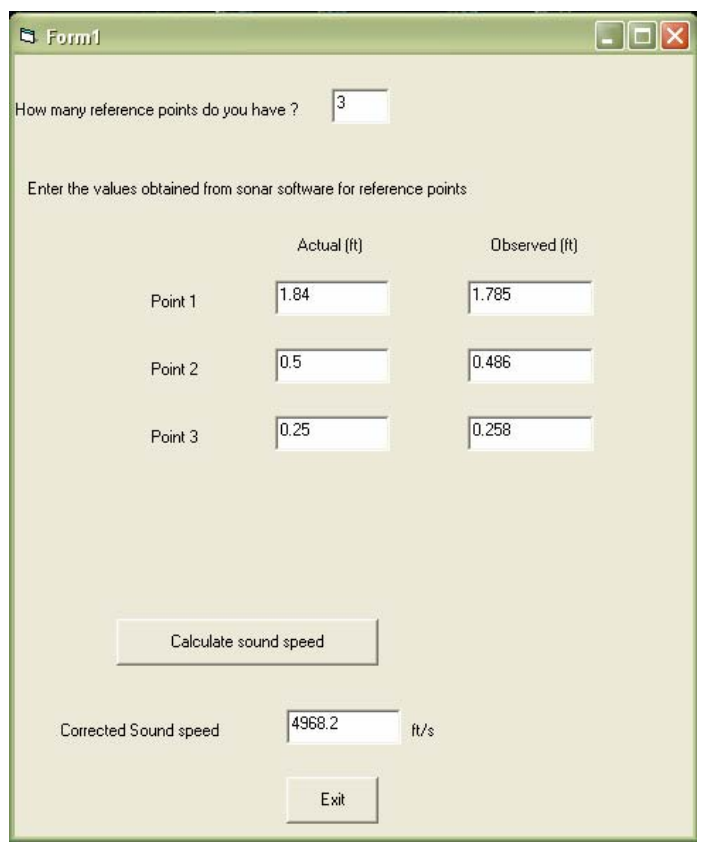

Figure 75: Screen shot of the software to generate the correct sound speed for different density and temperature of solution.

The reference heights were measure at $1500 \mathrm{~m} / \mathrm{s}$ or $4921.3 \mathrm{ft} / \mathrm{s}$ when drum was filled with tap water and following table was obtained. Actual, observed, and relative errors were calculated for each height for different temperatures (Table 14). Precise tape measurements were taken for the actual distances were and shown to be accurate within $1 \%$.

Table 14: Actual, Observed, and Relative Error in Heights at Three Temperatures at Default Sound Speed.

\begin{tabular}{|l|c|c|c|c|c|c|c|c|}
\hline \multirow{2}{*}{ Heights of } & \multicolumn{2}{|c|}{$\begin{array}{c}\text { Actual } \\
\text { Measurements }\end{array}$} & \multicolumn{2}{c|}{$25^{\circ} \mathrm{C}$} & \multicolumn{2}{c|}{$30^{\circ} \mathrm{C}$} & \multicolumn{2}{c|}{$35^{\circ} \mathrm{C}$} \\
\cline { 2 - 10 } & (in) & $(\mathrm{ft})$ & $\begin{array}{c}\text { Observed } \\
(\mathrm{ft})\end{array}$ & $\begin{array}{c}\text { Relative } \\
\text { Error }\end{array}$ & $\begin{array}{c}\text { Observed } \\
(\mathrm{ft})\end{array}$ & $\begin{array}{c}\text { Relative } \\
\text { Error }\end{array}$ & $\begin{array}{c}\text { Observed } \\
(\mathrm{ft})\end{array}$ & $\begin{array}{c}\text { Relative } \\
\text { Error }\end{array}$ \\
\hline Sonar & 22.12 & 1.84 & 1.811 & $1.5 \%$ & 1.811 & $1.5 \%$ & 1.785 & $2.9 \%$ \\
\hline Cube & 6.00 & 0.50 & 0.499 & $0.2 \%$ & 0.499 & $0.2 \%$ & 0.486 & $2.8 \%$ \\
\hline Rectangle & 3.00 & 0.25 & 0.249 & $0.4 \%$ & 0.249 & $0.4 \%$ & 0.236 & $5.6 \%$ \\
\hline
\end{tabular}




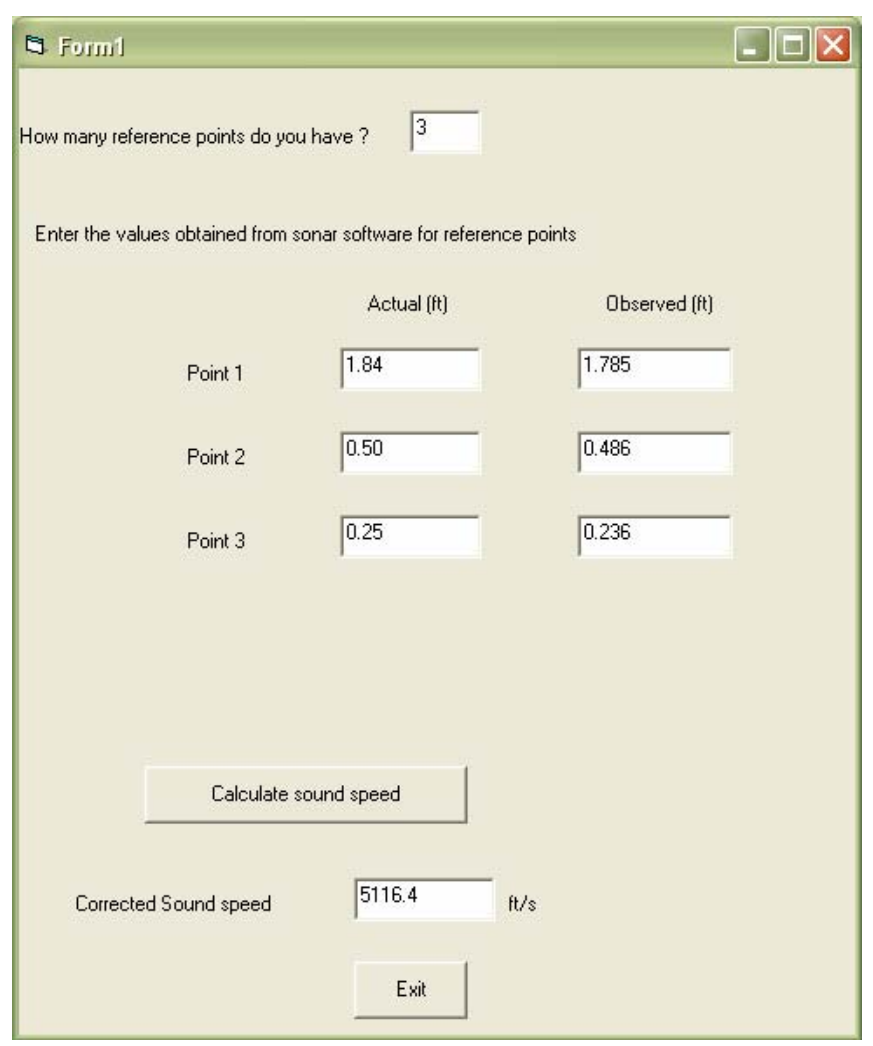

Figure 76: Correct sound speed generated by software for $35^{\circ} \mathrm{C}$ water.

The resultant sound speed in $35^{\circ} \mathrm{C}$ water was fed into the sonar software and Table 15 was generated.

Table 15: Actual, Observed and Relative Error in Heights at Corrected Sound Speed.

\begin{tabular}{|c|c|c|c|c|}
\hline \multirow{2}{*}{ Heights } & \multicolumn{2}{|c|}{ Actual Measurements } & \multicolumn{2}{c|}{ Observed Measurements at $35^{\circ} \mathrm{C}$} \\
\cline { 2 - 5 } & (in) & (ft) & $\begin{array}{c}\text { Observed } \\
(5116.41 \mathrm{ft} / \mathrm{s})\end{array}$ & Relative Error \\
\hline Sonar & 22.12 & 1.84 & 1.816 & $1.3 \%$ \\
\hline Cube & 6.00 & 0.50 & 0.505 & $1 \%$ \\
\hline Rectangle & 3.00 & 0.25 & 0.249 & $0.04 \%$ \\
\hline
\end{tabular}

Using default sound speed, measurements for sonar, cube, and rectangle (placed in the drum) were recorded as the density and the temperature of the solution was varied. These observed values were fed into the software written in V.B. 6 to generate correct 
sound speed. Table 16 summarizes the observed values (from sonar) at default and the corrected sound speed for the solution having $\rho=1.1,1.2,1.3$, and $1.4 \mathrm{~g} / \mathrm{cm}^{3}$ at three temperatures. When default sound speed $(4921.3 \mathrm{ft} / \mathrm{s})$ was used huge amount of relative error was observed but after inputting corrected sound speed from the software this error was minimized.

Table 16: Actual, Observed, and Relative Error in Heights for Solutions of Different Densities at $25^{\circ} \mathrm{C}$ with Default and Corrected Sound Speed.

\begin{tabular}{|c|c|c|c|c|c|c|c|c|}
\hline \multirow{2}{*}{$\begin{array}{l}\text { Tem } \\
\text { perat } \\
\text { ure }\end{array}$} & \multirow{2}{*}{$\begin{array}{l}\text { Density } \\
\left(\mathrm{g} / \mathrm{cm}^{3}\right)\end{array}$} & \multirow{2}{*}{$\begin{array}{c}\text { Sound } \\
\text { speed } \\
(\mathrm{ft} / \mathrm{s})\end{array}$} & \multicolumn{2}{|c|}{$\begin{array}{l}\text { Height of sonar } \\
\qquad(1.84 \mathrm{ft} .)\end{array}$} & \multicolumn{2}{|c|}{$\begin{array}{l}\text { Height of cube } \\
\qquad(0.50 \mathrm{ft} .)\end{array}$} & \multicolumn{2}{|c|}{$\begin{array}{c}\text { Height of rectangle } \\
(0.25 \mathrm{ft} .)\end{array}$} \\
\hline & & & $\begin{array}{c}\text { Observe } \\
\mathrm{d}(\mathrm{ft})\end{array}$ & $\begin{array}{l}\text { Relativ } \\
\text { e error }\end{array}$ & $\begin{array}{c}\text { Observe } \\
\mathrm{d}(\mathrm{ft})\end{array}$ & $\begin{array}{l}\text { Relativ } \\
\text { e error }\end{array}$ & $\begin{array}{c}\text { Observe } \\
\mathrm{d}(\mathrm{ft})\end{array}$ & $\begin{array}{l}\text { Relativ } \\
\text { e error }\end{array}$ \\
\hline \multirow{8}{*}{$25^{\circ} \mathrm{C}$} & \multirow{2}{*}{$\rho=1.1$} & $\mathrm{D}=4921.3$ & 1.732 & $5.8 \%$ & 0.472 & $5.6 \%$ & 0.223 & $10 \%$ \\
\hline & & $C=5319.5$ & 1.844 & $0.2 \%$ & 0.496 & $0.8 \%$ & 0.255 & $2 \%$ \\
\hline & \multirow{2}{*}{$\rho=1.2$} & $\mathrm{D}=4921.3$ & 1.667 & $10.8 \%$ & 0.446 & $10.8 \%$ & 0.210 & $16 \%$ \\
\hline & & $\mathrm{C}=5602.6$ & 1.838 & $0.1 \%$ & 0.490 & $2 \%$ & 0.254 & $1.6 \%$ \\
\hline & \multirow{2}{*}{$\rho=1.3$} & $\mathrm{D}=4921.3$ & 1.549 & $15 \%$ & 0.446 & $10 \%$ & 0.420 & $16 \%$ \\
\hline & & $C=5626.7$ & 1.836 & $0.2 \%$ & 0.495 & $1 \%$ & 0.249 & $0.04 \%$ \\
\hline & \multirow{2}{*}{$\rho=1.4$} & $\mathrm{D}=4921.3$ & 1.509 & $17 \%$ & 0.459 & $8.2 \%$ & 0.184 & $26.4 \%$ \\
\hline & & $\mathrm{C}=6016.1$ & 1.845 & $0.2 \%$ & 0.497 & $0.6 \%$ & 0.254 & $1.6 \%$ \\
\hline
\end{tabular}

In the Table 16 "D" represents the default sound speed $(1500 \mathrm{~m} / \mathrm{s})$ and "C" represents the corrected sound speed obtained from the software shown in Figure 26. Effect on sound speed at different temperature was also studied. Also software was tested for all the temperature for its accuracy in generating correct sound speed. Tables 17 and 18 were obtained when temperature was increased to 30 and $35^{\circ} \mathrm{C}$. The maximum temperature kept was $35^{\circ} \mathrm{C}$ as, this is the maximum temperature which is expected in HLW tanks at Hanford. 
Table 17: Actual, Observed, and Relative Error in Heights for Solutions of Different Densities at $30^{\circ} \mathrm{C}$ with Default and Corrected Sound Speed.

\begin{tabular}{|c|c|c|c|c|c|c|c|c|}
\hline \multirow{2}{*}{$\begin{array}{l}\text { Tem } \\
\text { perat } \\
\text { ure }\end{array}$} & \multirow{2}{*}{$\begin{array}{l}\text { Density } \\
\left(\mathrm{g} / \mathrm{cm}^{3}\right)\end{array}$} & \multirow{2}{*}{$\begin{array}{l}\text { Sound } \\
\text { speed } \\
(\mathrm{ft} / \mathrm{s})\end{array}$} & \multicolumn{2}{|c|}{$\begin{array}{l}\text { Height of sonar } \\
\text { (1.84 ft.) }\end{array}$} & \multicolumn{2}{|c|}{$\begin{array}{l}\text { Height of cube } \\
(0.50 \mathrm{ft} .)\end{array}$} & \multicolumn{2}{|c|}{$\begin{array}{c}\text { Height of rectangle } \\
(0.25 \mathrm{ft} .)\end{array}$} \\
\hline & & & $\begin{array}{l}\text { Observe } \\
\mathrm{d}(\mathrm{ft})\end{array}$ & $\begin{array}{l}\text { Relativ } \\
\text { e error }\end{array}$ & $\begin{array}{c}\text { Observe } \\
\mathrm{d}(\mathrm{ft})\end{array}$ & $\begin{array}{l}\text { Relativ } \\
\text { e error }\end{array}$ & $\begin{array}{c}\text { Observe } \\
\mathrm{d}(\mathrm{ft})\end{array}$ & $\begin{array}{l}\text { Relativ } \\
\text { e error }\end{array}$ \\
\hline \multirow{8}{*}{$30^{\circ} \mathrm{C}$} & \multirow{2}{*}{$\rho=1.1$} & $\mathrm{D}=4921.3$ & 17.06 & $7.2 \%$ & 0.459 & $8.2 \%$ & 0.223 & $10 \%$ \\
\hline & & $\mathrm{C}=5395.2$ & 1.842 & $1 \%$ & 0.495 & $1 \%$ & 0.245 & $2 \%$ \\
\hline & \multirow{2}{*}{$\rho=1.2$} & $\mathrm{D}=4921.3$ & 1.627 & $11.6 \%$ & 0.446 & $10.8 \%$ & 0.223 & $6.8 \%$ \\
\hline & & $\mathrm{C}=5533.2$ & 1.842 & $0.1 \%$ & 0.502 & $0.4 \%$ & 0.501 & $0.2 \%$ \\
\hline & \multirow{2}{*}{$\rho=1.3$} & $\mathrm{D}=4921.3$ & 1.562 & $15 \%$ & 0.433 & $13 \%$ & 0.238 & $5 \%$ \\
\hline & & $\mathrm{C}=5549.8$ & 1.844 & $0.2 \%$ & 0.502 & $0.4 \%$ & 0.252 & $0.8 \%$ \\
\hline & \multirow{2}{*}{$\rho=1.4$} & $\mathrm{D}=4921.3$ & 1.496 & $18.7 \%$ & 0.433 & $13 \%$ & 0.171 & $31 \%$ \\
\hline & & $\mathrm{C}=6310.2$ & 1.844 & $0.2 \%$ & 0.503 & $0.6 \%$ & 0.252 & $0.8 \%$ \\
\hline
\end{tabular}

Table 18: Actual, Observed, and Relative Error in Heights for Solutions of Different Densities at $35^{\circ} \mathrm{C}$ with Default and Corrected Sound Speed.

\begin{tabular}{|c|c|c|c|c|c|c|c|c|}
\hline \multirow{2}{*}{$\begin{array}{l}\text { Tem } \\
\text { perat } \\
\text { ure }\end{array}$} & \multirow{2}{*}{$\begin{array}{l}\text { Density } \\
\left(\mathrm{g} / \mathrm{cm}^{3}\right)\end{array}$} & \multirow{2}{*}{$\begin{array}{c}\text { Sound } \\
\text { speed } \\
(\mathrm{ft} / \mathrm{s})\end{array}$} & \multicolumn{2}{|c|}{$\begin{array}{l}\text { Height of sonar } \\
\qquad(1.84 \mathrm{ft} .)\end{array}$} & \multicolumn{2}{|c|}{$\begin{array}{c}\text { Height of cube } \\
(0.50 \mathrm{ft} .)\end{array}$} & \multicolumn{2}{|c|}{$\begin{array}{c}\text { Height of rectangle } \\
(0.25 \mathrm{ft} .)\end{array}$} \\
\hline & & & $\begin{array}{c}\text { Observe } \\
\mathrm{d}(\mathrm{ft})\end{array}$ & $\begin{array}{l}\text { Relativ } \\
\text { e error }\end{array}$ & $\begin{array}{c}\text { Observe } \\
\mathrm{d}(\mathrm{ft})\end{array}$ & $\begin{array}{l}\text { Relativ } \\
\text { e error }\end{array}$ & $\begin{array}{c}\text { Observe } \\
\mathrm{d}(\mathrm{ft})\end{array}$ & $\begin{array}{l}\text { Relativ } \\
\text { e error }\end{array}$ \\
\hline \multirow{8}{*}{$35^{\circ} \mathrm{C}$} & \multirow{2}{*}{$\rho=1.1$} & $\mathrm{D}=4921.3$ & 1.706 & $7.2 \%$ & 0.472 & $5.6 \%$ & 0.223 & $10 \%$ \\
\hline & & $\mathrm{C}=5346.0$ & 1.837 & $0.1 \%$ & 0.499 & $0.2 \%$ & 0.249 & $0.4 \%$ \\
\hline & \multirow{2}{*}{$\rho=1.2$} & $\mathrm{D}=4921.3$ & 1.640 & $10.8 \%$ & 0.433 & $13.4 \%$ & 0.210 & $16 \%$ \\
\hline & & $C=5687.6$ & 1.838 & $0.1 \%$ & 0.501 & $0.2 \%$ & 0.248 & $0.8 \%$ \\
\hline & \multirow{2}{*}{$\rho=1.3$} & $\mathrm{D}=4921.3$ & 1.562 & $15 \%$ & 0.420 & $16 \%$ & 0.210 & $16 \%$ \\
\hline & & $\mathrm{C}=5838.1$ & 1.873 & $0.1 \%$ & 0.500 & $0.0 \%$ & 0.250 & $0.0 \%$ \\
\hline & \multirow{2}{*}{$\rho=1.4$} & $\mathrm{D}=4921.3$ & 1.509 & $17 \%$ & 0.446 & $10.8 \%$ & 0.171 & $31 \%$ \\
\hline & & $\mathrm{C}=6237.6$ & 1.839 & $0.0 \%$ & 0.499 & $0.2 \%$ & 0.245 & $2 \%$ \\
\hline
\end{tabular}




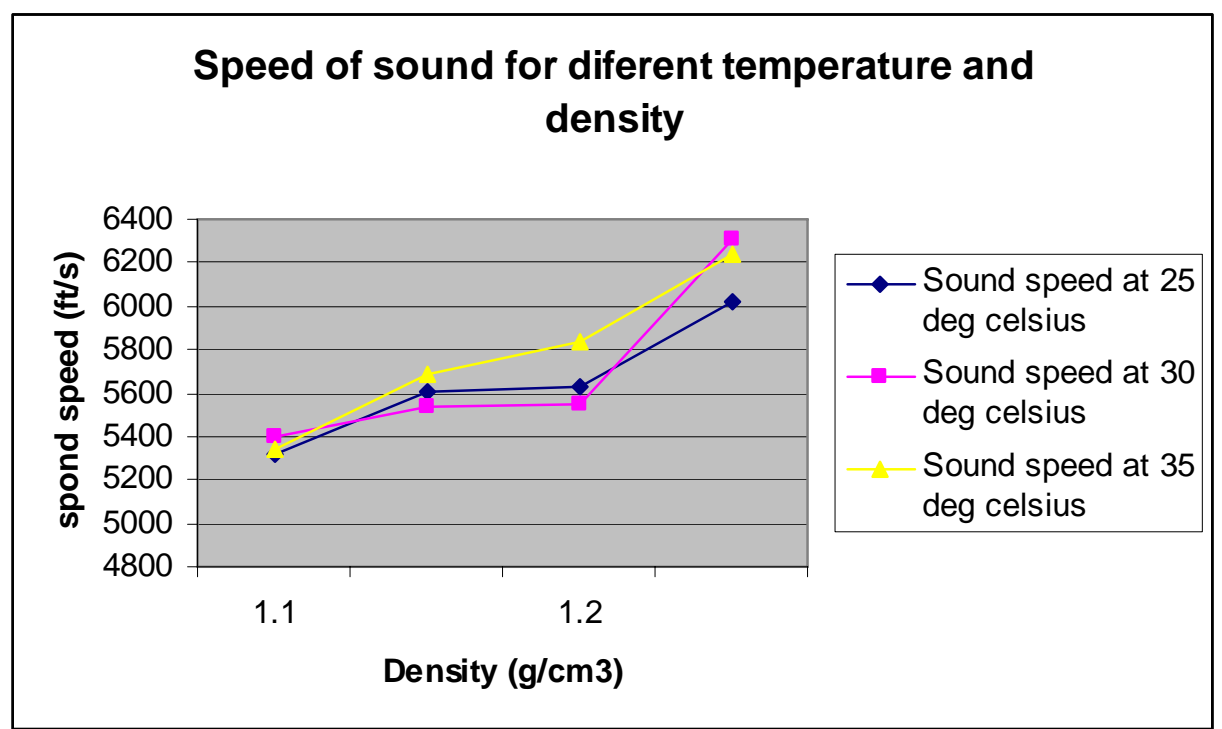

Figure 77: Plot of change in sound speed at different temperature when density of the water is increased from $0.99 \mathrm{~g} / \mathrm{cm}^{3}$ to $1.4 \mathrm{~g} / \mathrm{cm}^{3}$.

From the graph of the Figure 77 it can be analyzed that sound speed increases with density and it shows an abnormal rise at $1.4 \mathrm{~g} / \mathrm{cm}^{3}$. Also the sound speed increases with temperature. The trend is almost linear with temperature. From the graph it can also be concluded that sound speed in the actual HLW tanks will vary in the range of $5200 \mathrm{ft} / \mathrm{s}$ to $6400 \mathrm{ft} / \mathrm{s}$ as the density and the temperature will vary in the same range as discussed above. 


\section{Chapter 4}

\section{CONCLUSIONS AND POTENTIAL FUTURE WORK}

Following inferences can be drawn from the results of the experiments conducted

1. Profiling sonar can be used effectively for real-time monitoring of the solid-liquid interface.

2. Profiling sonar images several square meters of interface of solids surface in the large HLW tank. The accuracy of the height of the interface is $\pm 0.36 \mathrm{~cm}$ or better at a $2 \mathrm{~m}$ range.

3. Even with $30 \%$ by weight of solids suspended in the liquid sonar is able to accurately measure the solid-liquid interface with $\pm 0.91 \mathrm{~cm}$ or better at a $2 \mathrm{~m}$ range..

4. Sonar can detect with accuracy of $\pm 0.36 \mathrm{~cm}$ (at $2 \mathrm{~m}$ range) settled solids having a density of $4 \%$ greater than the fluid in which they are immersed. These lighter solids are easily suspended and can take some time to resettle.

5. To have the accuracy of $\pm 0.36 \mathrm{~cm}$ (at $2 \mathrm{~m}$ range) profiling sonar head should be at least $7.2 \mathrm{in.}$ above the interface and at least $10 \mathrm{in}$. away from a wall to image accurately.

6. Based upon the material selection for the sonar head (Titanium hull) and its cable (Polyurethane outer coating), caustic solution $(\mathrm{pH}>14)$ does not have an effect on the sonar's ability to generate images and function properly.

7. Sonar's imaging is not effected by changing either the solution density or the temperature. Only measurements between the relative points are effected. This is due to the change in sound speed with density and temperature. The error in 
measurements can be corrected by putting the correct sound speed in the sonar software.

\section{Future Scope}

Further studies are warranted to verify the capability of the sonar to detect the solid-liquid interface for volume larger than 1205.16 cubic feet (demonstrated here). To be considered as a solution for high-level waste monitoring, the system would have to first be tested: in larger volume of caustic solution as HLW tanks have 615752.16 cubic feet volume per tank. To best simulate the field conditions, the following test-bed is recommended

1. The exact placement of the sonar in the HLW tank is not know (the riser from which sonar is going to be deployed is not known), the effect of objects near to sonar head (less than $2 \mathrm{ft}$. from sonar head) when the range is $20 \mathrm{ft}$ or more needs to be studied.

2. Sound speed in sonar should be corrected before taking measurements for specific density or temperature change.

3. Sonar performance should also be tested when multiple density solutions are present as each solution will give rise to different sound speed and may result in faulty sonar measurements.

4. There may be formation of colloids in the caustic solution due to pumping and mixing. It would be useful to study the effect of colloids on sonar performance.

5. Effect of electromagnetic wave on the sonar circuitry should be studied as it may effect the working of sonar transducer and other electronic components. 
6. Since the image generated by sonar is in $2-\mathrm{D}$, a software to visualize tank bottom in 3-D is necessary. 


\section{BIBLIOGRAPHY}

[1] Hunt, F.V., Electrostatics, Chapter 1, John Wiley \& Sons Inc., New York, 1954

[2] Hill, S., Mystery of Titanic: A Case of Brittle Fracture, Materials worlds, 1996, Volume 4, pages 334-335.

[3] Atkins, P., Tutorial Introduction and Historical Overview of the need for Heading sensors in sonar applications, IEE Colloquium, 1995,Volume 4, pages 1-28

[4] Wade, G., A History of Acoustic Imaging, Acoustic Imaging, 1987, Volume 12, pages 1-28.

[5] Hayes, H.C., Detection of Submarines, Proc. Am. Phil. Soc., 1920, pages 1-59

[6] Klien, E., Underwater Sound and Naval Acoustical Research and Applications before 1939, J. Acoustic Society American., 1968, Volume 33 pages 43-93

[7] Harrison ,T., L., Sonar Engineering Handbook. Peninsula publishing, 1992.

[8] Morgan, L., A., Shanks, W., C., Lovalov, D., Wering, M., Lee, G., Stephenson, W., J., Johnson, S., Y., Mapping the Floors of Yellow Stones Lake: New Discoveries from Higher-Resolution Sonar Imaging, Seismic-Reflection Profiling, and Submersible Studies, U.S. Geographical survey, 2001, Denver Federal Center.

[9] Doug, L., Edward, S., William, G., Multi beam scatter comparisons: A Comprehensive Analysis of Comparative Systems and Manufacturers for Water Depths between 6.0 - 6000 meters, National Marine Fisheries Service, 2004, C.A.

[10] Abraham, D., A., Willett, P., K., Active Sonar Detection in Shallow Water Using the Page Test, IEEE Journal of Oceanic Engineering, 2002, Volume. 27, pages 3546.

[11] Shin, B., N., Kil, H., D., Wayland, F., R., Active Impulse Echo Discrimination in Shallow Water by Mapping Target Physics-Derived Features to Classifiers, IEEE Journal of Organic Engineering, 1997, Volume 22, pages 66-80

[12] Mindell, D., A., Bingham, B., A High Frequency, Narrow-Beam Sub Bottom Profiler for Archeological Survey, Massachusetts Institute of Technology, 1997, Research Group in Deep Sea Archaeology.

[13] Schock, S., G., Tellier, A., Wulf, J., Sara, J., Ericksen, M., Buried Object Scanning Sonar, IEEE Journal of Oceanic Engineering, 2001, Volume 26, pages 677-689 
[14] Kim, K., M., Lee, C., Youn, D., H., Adaptive Processing for Enhanced CFAR Technique Detecting Performance in Active Sonar Systems, IEEE Journal of Oceanic Engineering, 1998, Volume 22, pages 693-700.

[15] Elfes, A., Sonar Based Real-World Mapping and Navigation, IEEE Trans. Robotics Automation, 1987, Volume 3, pages 249-265.

[16] Kuc, R., Viard, B., V., A physically-based navigation strategy for sonar-guided vehicles, International Journal of Robotics, 1991, pages 75-87.

[17] Barshan, B., Ayrulu, B., Comparative analysis of different approaches to target differentiation and localization with sonar, Department of Electrical Engineering, 2002, Bilkent University, Turkey.

[18] Schalder, M., D., Willem, B., M., An Innovative Approach to In Service Above Ground Storage Tank Testing, American Society of Mechanical Engineering, 1993, Volume 54, pages 33-38.

[19] Waite, A., D., Sonar For Practicing Engineers, $3^{\text {rd }}$ Edition, John Wiley \& sons Inc., New York, 2002.

[20] Urick., R., J., Principles of Underwater Sound, $2^{\text {nd }}$ Edition, Chapter 3 and 4 McGraw-Hill Book Company, New York, 1975.

[21] Urick, R., J, Generalized Form of Sonar Equations, Journal of Acoustic Society of America, 1962, pages 34-547.

[22] Harrision, C., H., Robins, A., J., Modelling Acoustic Propogation In the Time Domain, IEEE Oceanic Engineering Society, 1998, Volume 1, pages 1-5

[23] Harrison, C., H., Robins, A., J., Cowley, A., A Comparison of Modelled Time Series with Measurements, IEEE Oceanic Engineering Society, 1998, Volume 1, pages 6-9

[24] Mazumdar, A., Roelant, D., Srivastava, R., Near-Real-Time Solid-Liquid Interface Monitor, DE-FG01-03EW15334, Hemispherical Center for Environmental Technology, Florida International University, 2003, Florida.

[25] Sood, N., Roelant, D., Srivastava, R., Monitoring The Solid Liquid Interface In Tanks using Profiling Sonar And 3-D Visualization Techniques, SPIE-International Society of for Optical Engineering, 2005, Volume 13, page 70-83. 
[26] Imagenex 881A manual version 2.2 software, 2003, operating manual available at www.Imagenex.com 


\section{APPENDICES}

\section{Appendix A}

Table 1: Imagenex model $881 \mathrm{~A}$ sonar hardware specifications.

\begin{tabular}{|c|c|}
\hline \multicolumn{2}{|c|}{ Hardware Specifications } \\
\hline Frequency & $\begin{array}{l}310 \mathrm{kHz}, 675 \mathrm{kHz} \text {, or } 1 \mathrm{MHz} \text { (default settings) } \\
\text {-Other frequencies can be selected through programmable } \\
\text { software configurations } \\
\text { Tunable from } 280 \mathrm{kHz} \text { to } 1.1 \mathrm{MHz} \text { in } 5 \mathrm{kHz} \text { steps }\end{array}$ \\
\hline Transducer & Imaging type, fluid compensated \\
\hline $\begin{array}{l}\text { Transducer Beam } \\
\text { Width }\end{array}$ & $\begin{array}{l}310 \mathrm{kHz}: 4^{\circ} \times 40^{\circ} \\
675 \mathrm{kHz}: 1.8^{\circ} \times 20^{\circ} \\
1 \mathrm{MHz}: 0.9^{\circ} \times 10^{\circ}\end{array}$ \\
\hline Range Resolution & $\begin{array}{l}1 \mathrm{~m}-4 \mathrm{~m}: 2 \mathrm{~mm}\left(0.08^{\prime \prime}\right) \\
5 \mathrm{~m} \& \text { up: } 10 \mathrm{~mm}\left(0.4^{\prime \prime}\right)\end{array}$ \\
\hline $\begin{array}{l}\text { Min. Detectable } \\
\text { Range }\end{array}$ & $150 \mathrm{~mm}(6 ")$ \\
\hline $\begin{array}{l}\text { Max. Operating } \\
\text { Depth }\end{array}$ & $1000 \mathrm{~m}$ and $3000 \mathrm{~m}$ available \\
\hline Max. Cable Length & $1000 \mathrm{~m}$ on typical twisted shielded pair (RS-485) \\
\hline Interface & RS-485 serial interface $@ 115.2$ kbps(or optional RS-232) \\
\hline Connector & $\begin{array}{l}\text { Side mounted, four conductor, wet malleable(Impulse MCBH- } \\
\text { 4-MP-SS) }\end{array}$ \\
\hline Power Supply & $20-36$ VDC. \\
\hline $\begin{array}{l}\text { Dimensions } \\
\text { (for both depth } \\
\text { ratings) }\end{array}$ & $79.4 \mathrm{~mm}(3.125 ")$ diameter x182 mm (7.125") \\
\hline $\begin{array}{r}\text { Weight : In Air } \\
\text { In Water }\end{array}$ & $\begin{array}{l}3000 \mathrm{~m} \text { unit: } 2 \mathrm{~kg}(4.4 \mathrm{lbs}) \\
3000 \mathrm{~m} \text { units } 1.1 \mathrm{~kg}(2.2 \mathrm{lbs})\end{array}$ \\
\hline Materials & $\begin{array}{l}3000 \text { m unit: Titanium, Polyurethane \& } 300 \text { series stainless } \\
\text { steel }\end{array}$ \\
\hline Finish & Hard Anodize \\
\hline
\end{tabular}




\section{Appendix B}

Table 1: Imagenex model 881A sonar software specifications.

\begin{tabular}{|l|l|}
\hline \multicolumn{2}{|l|}{ Software Specification : Win881A.exe } \\
\hline $\begin{array}{l}\text { Windows } \\
\text { Operating System }\end{array}$ & Windows \\
\hline Modes & Sector, Polar and Side Scan \\
\hline Range Scales & $\begin{array}{l}1 \mathrm{~m}, 2 \mathrm{~m}, 3 \mathrm{~m}, 4 \mathrm{~m}, 5 \mathrm{~m}, 10 \mathrm{~m}, 20 \mathrm{~m}, 30 \mathrm{~m}, 40 \mathrm{~m}, 50 \mathrm{~m}, 60 \mathrm{~m}, 80 \mathrm{~m}, \\
100 \mathrm{~m}, 150 \mathrm{~m}, 200 \mathrm{~m}\end{array}$ \\
\hline Train Angles & $0^{\circ}-357^{\circ}, 3^{\circ}$ increments \\
\hline $\begin{array}{l}\text { Sector Size: } \\
\text { Sector mode } \\
\text { Polar mode }\end{array}$ & $\begin{array}{l}0^{\circ}-180^{\circ}, 3^{\circ} \text { increments } \\
0^{\circ}-357^{\circ}, 3^{\circ} \text { increments }\end{array}$ \\
\hline Step Size & $\begin{array}{l}\text { Slow }\left(0.3^{\circ}\right), \text { Medium }\left(0.6^{\circ}\right), \text { Fast }\left(0.9^{\circ}\right), \text { Faster }\left(1.2^{\circ}\right), \\
\text { Fastest }\left(2.4^{\circ}\right)\end{array}$ \\
\hline Grid Types & Polar and Rectangular \\
\hline File format & (Filename). 81a \\
\hline $\begin{array}{l}\text { Recommended } \\
\text { Minimum Computer } \\
\text { Requirements: }\end{array}$ & $\begin{array}{l}100 \mathrm{MHz} \text { Pentium, } 16 \mathrm{MB} \text { RAM, } 1 \text { GB Hard Disk, } 800 \times 600 \mathrm{x} \\
256 \text { color graphics }\end{array}$ \\
\hline
\end{tabular}

Figure 3 shows an image generated by acoustic sonar when specifications used in Figure

1 and Figure 2 were fed into the software.

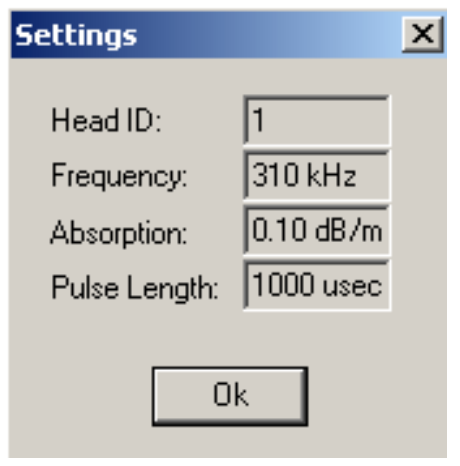

Figure 1: Selected settings for the sonar.

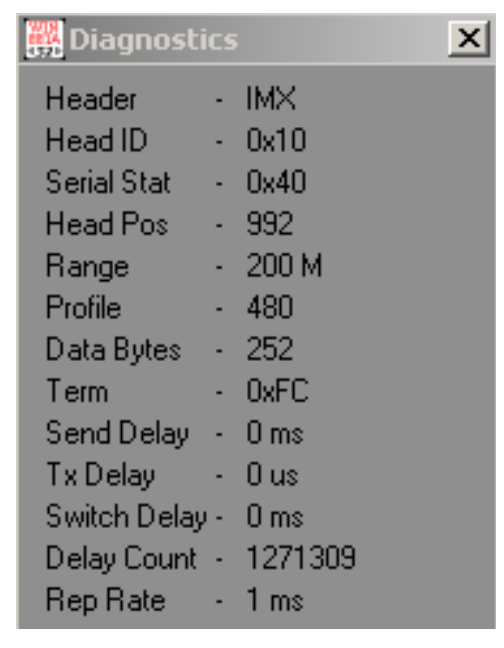

Figure 2: More selections for the settings. 


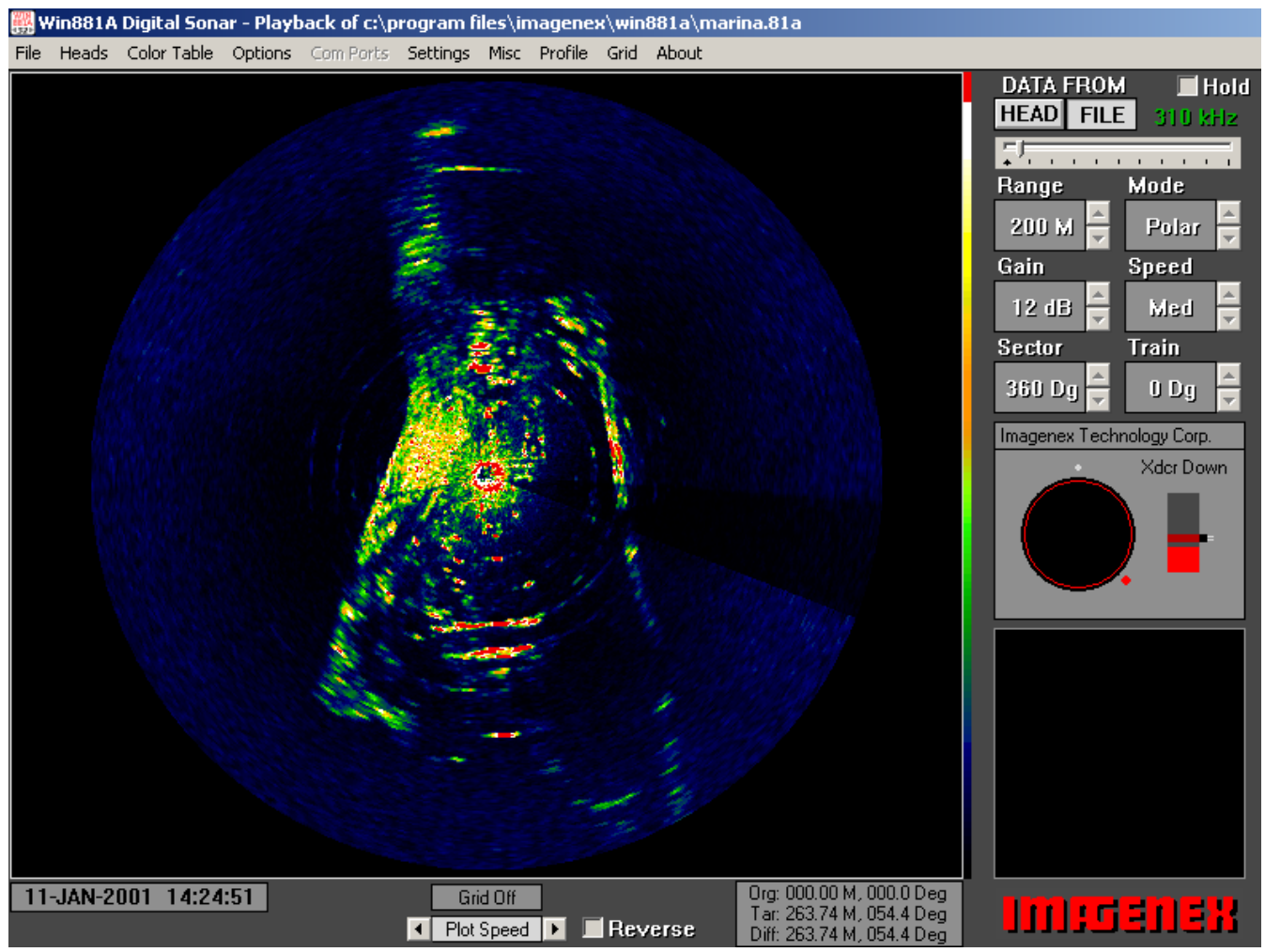

Figure 3: Screen shot of the displayed image with selected Parameters.

Figure 4 similar to Figure 3 but uses pixel zoom command that allows the user to have a closer view of any desired part of an image. 


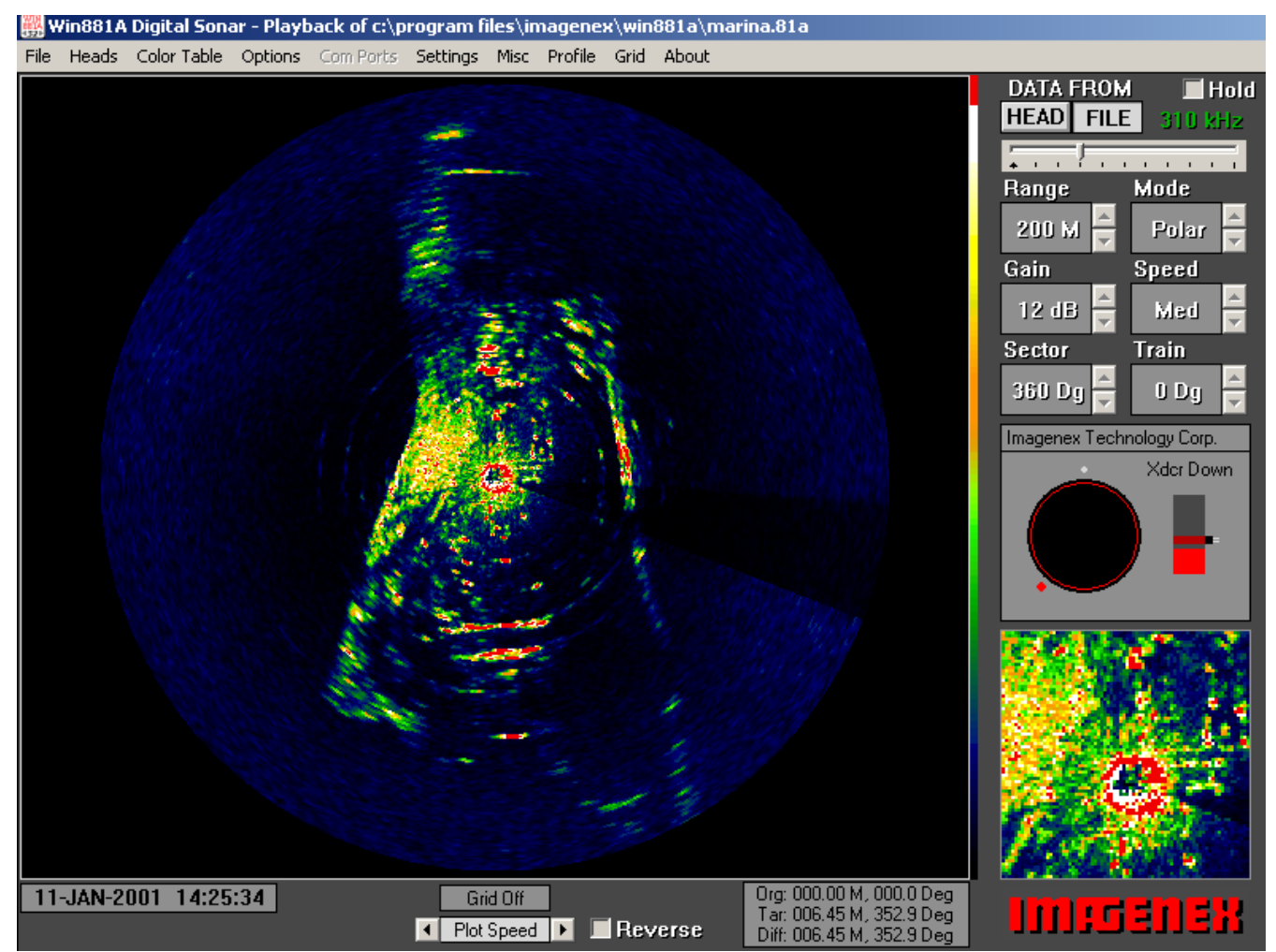

Figure 4: Screen shot of output image using pixel zoom command.

There are nine options available to view the output image generated, examples of 2 images are shown former one in grey scale (Figure 5) and latter one in brown-yellow color (Figure 6). 


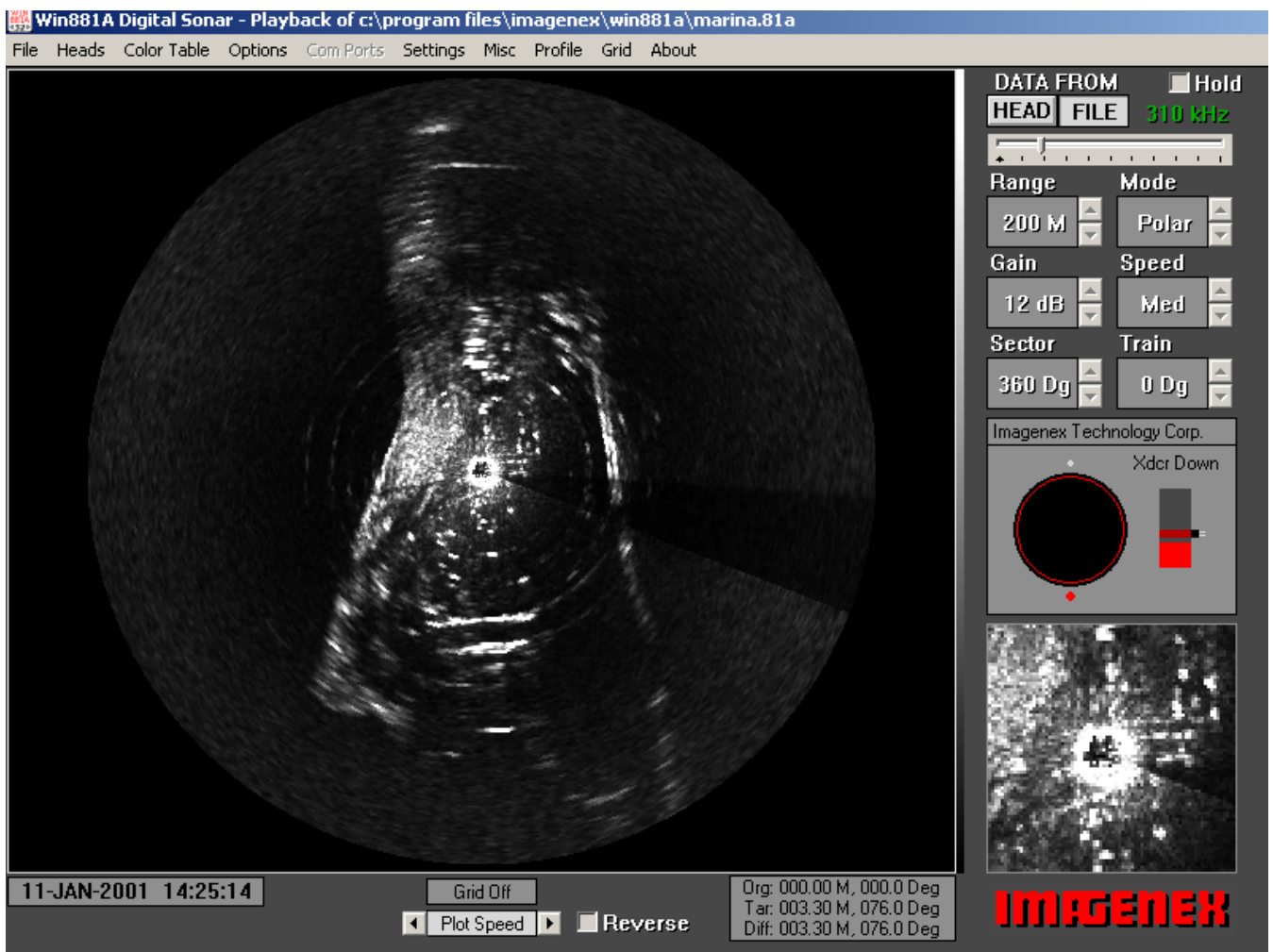

Figure 5: Output image displayed in grey scale color.

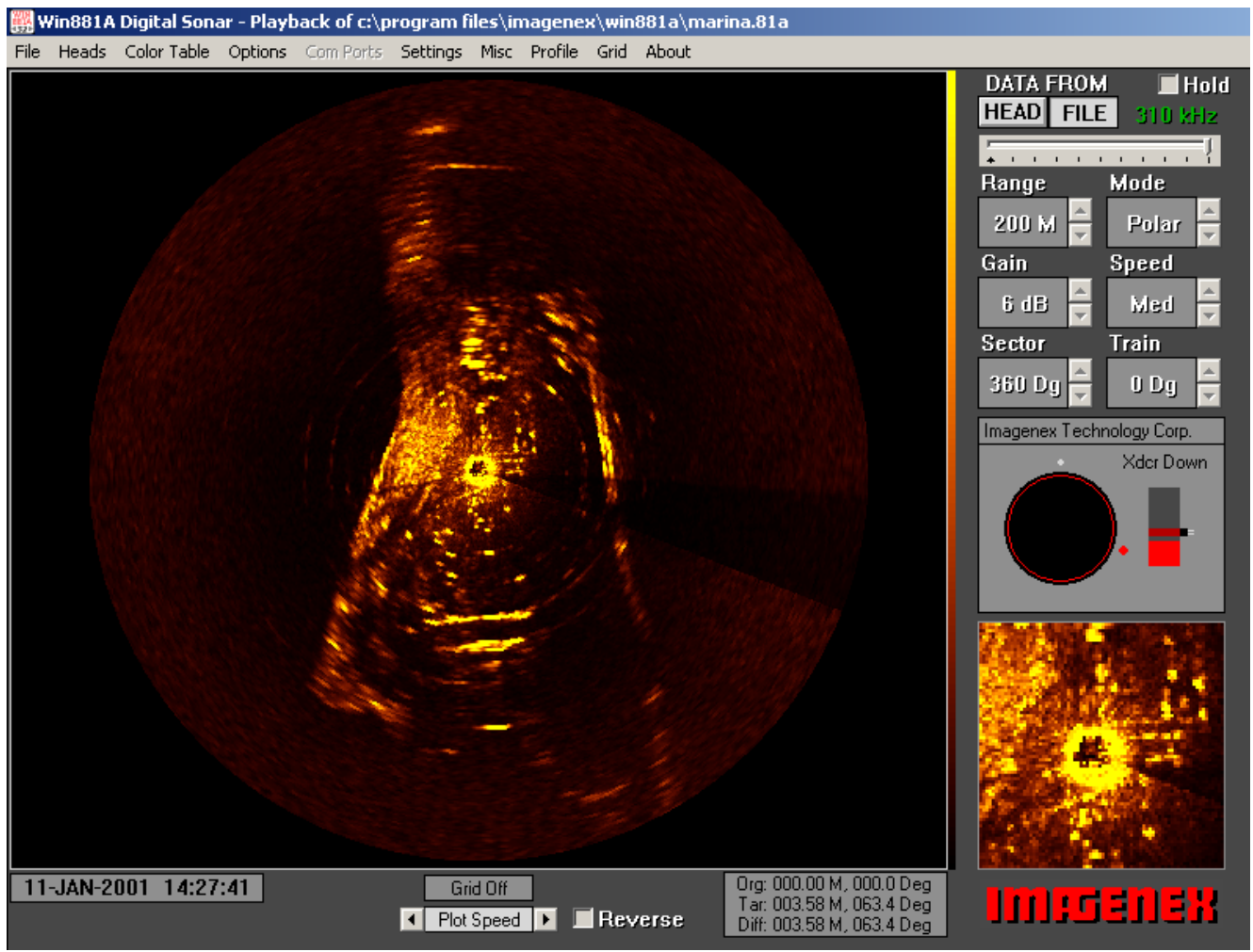

Figure 6: Output image generated in brown-yellow color. 
Figure 7 shows an image with Grid cells on, the grids can also be seen in the pixel zoom window that enables better tracking and detecting of the scanned matter.

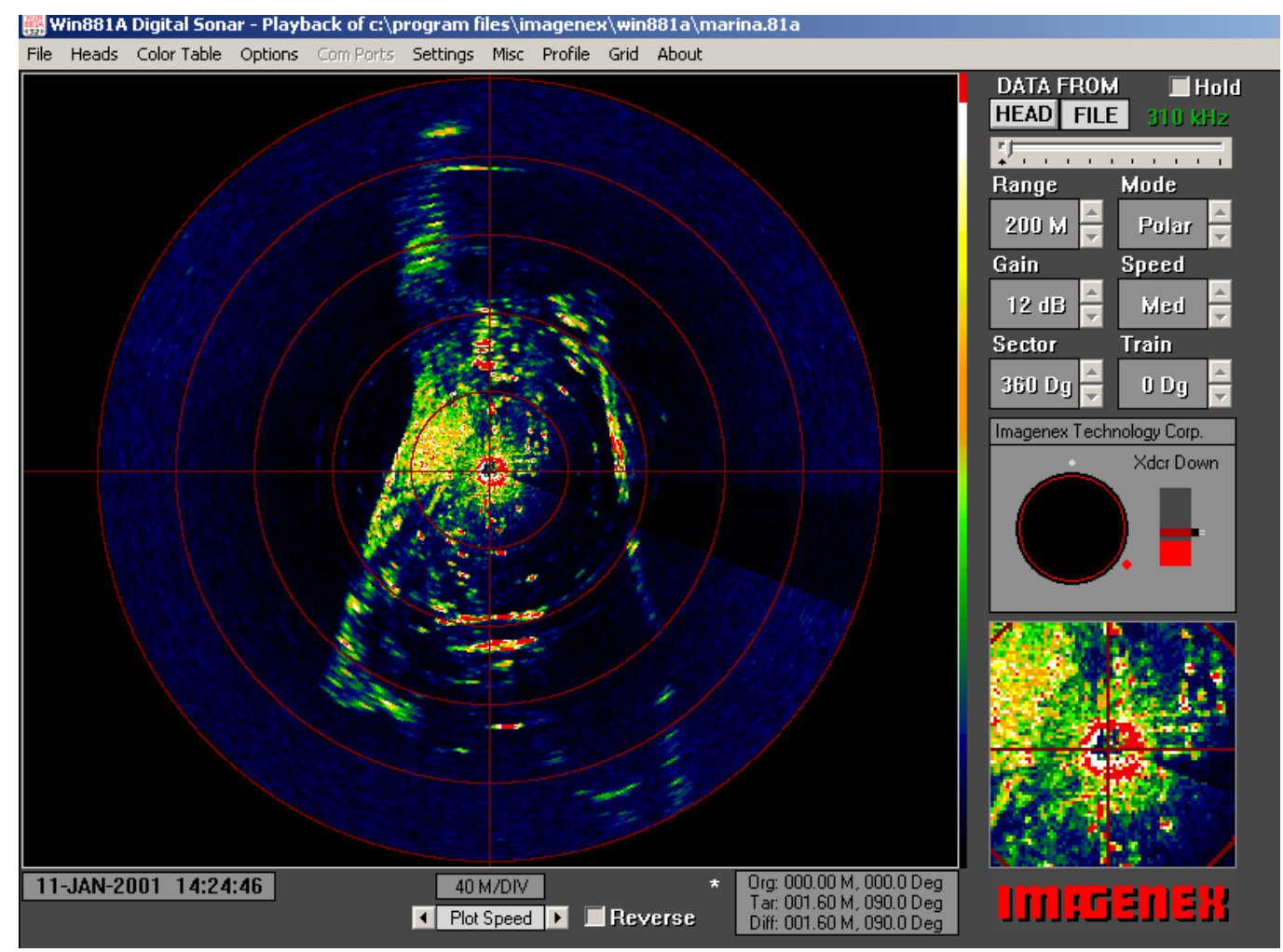

Figure 7: Screen shot of output image with grid on.

Figure 8 shows alternative representations of the grid cells. This representation is used when the sonar head is facing up (red color of the sensor on top). The diagram also includes the profile set up. 


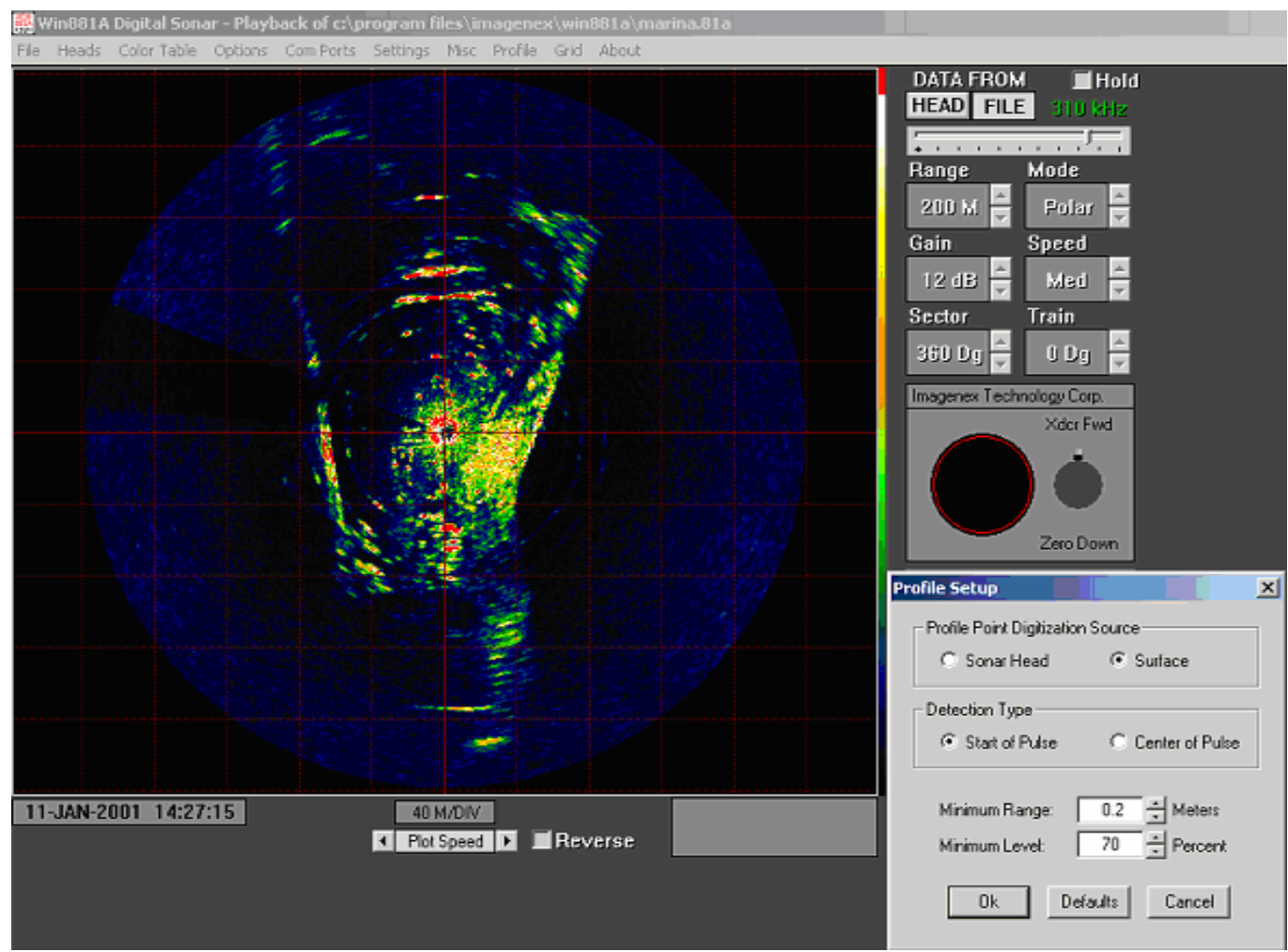

Figure 8: Screen Shot of Output Image showing alternative grid representation

Figure 9 show the image generated by sonar to identify the location of originating points that are used in the construction of the complete image. The image can also be adjusted to low-mix, medium-mix, and high-mix display modes. This property is used to generate points scanned by the sonar rather than having an complete image. 


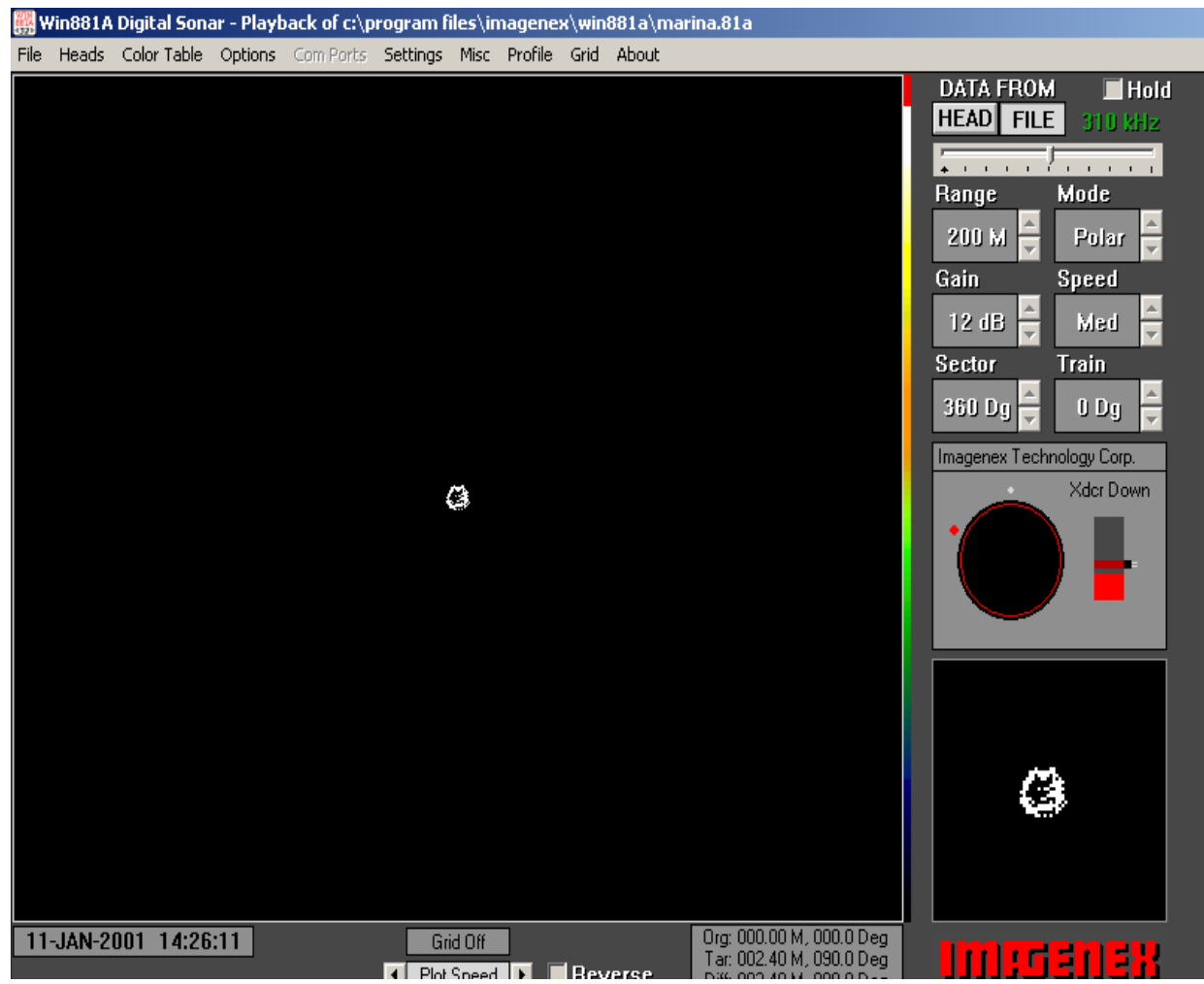

Figure 9: Output Image showing location of originating points

Figure 10 shows an example of another image (obtained from demo file). This image was generated in sector mode and the changes can be observed in Mode Gain, Range and Sector switches on the screen. 


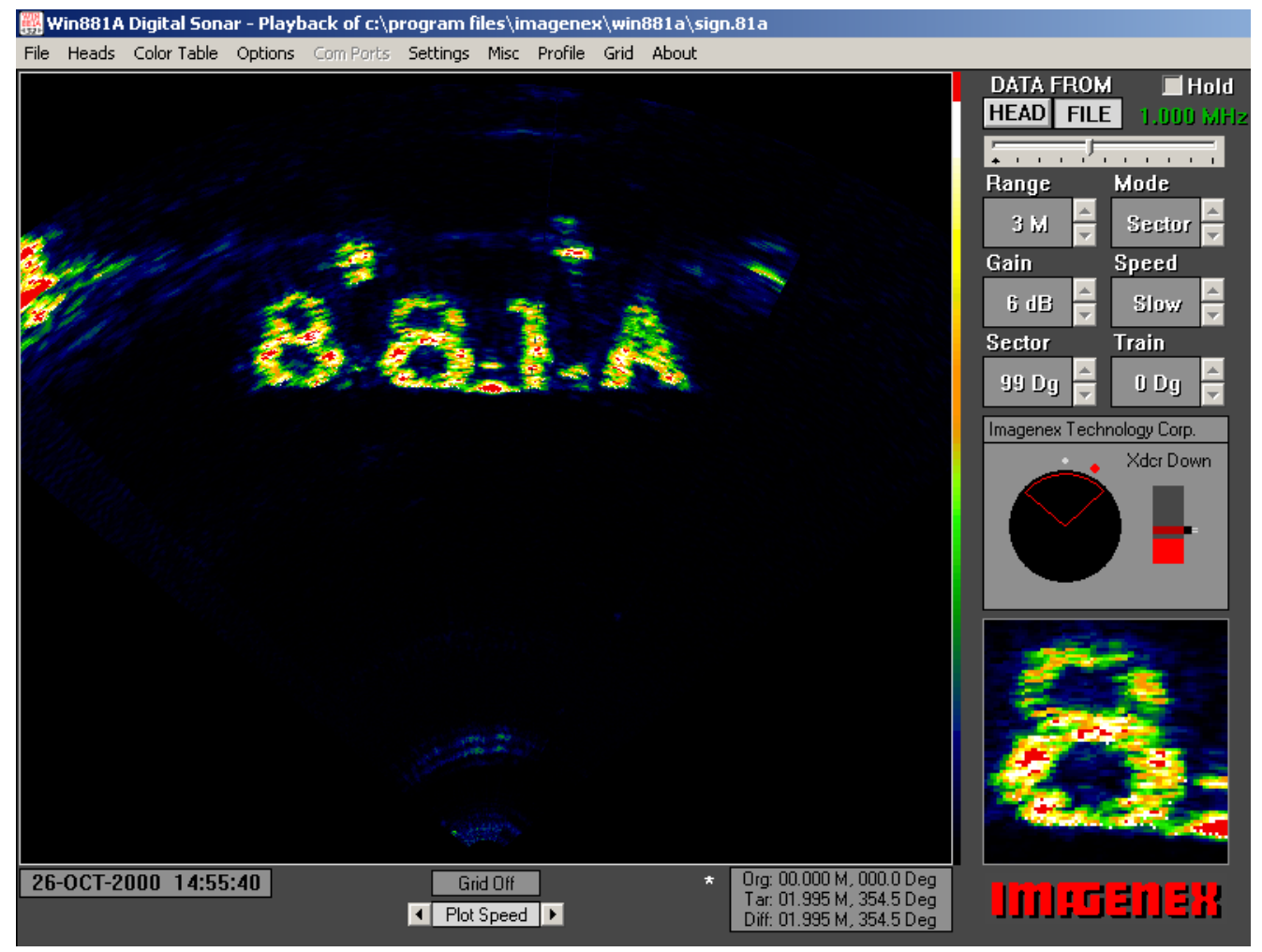

Figure 10: Screen shot of another output image generated in the sector mode

Figure 11 shows output image displaying two-cursor measurement that is used to calculate the distances between any two points on the screen. 


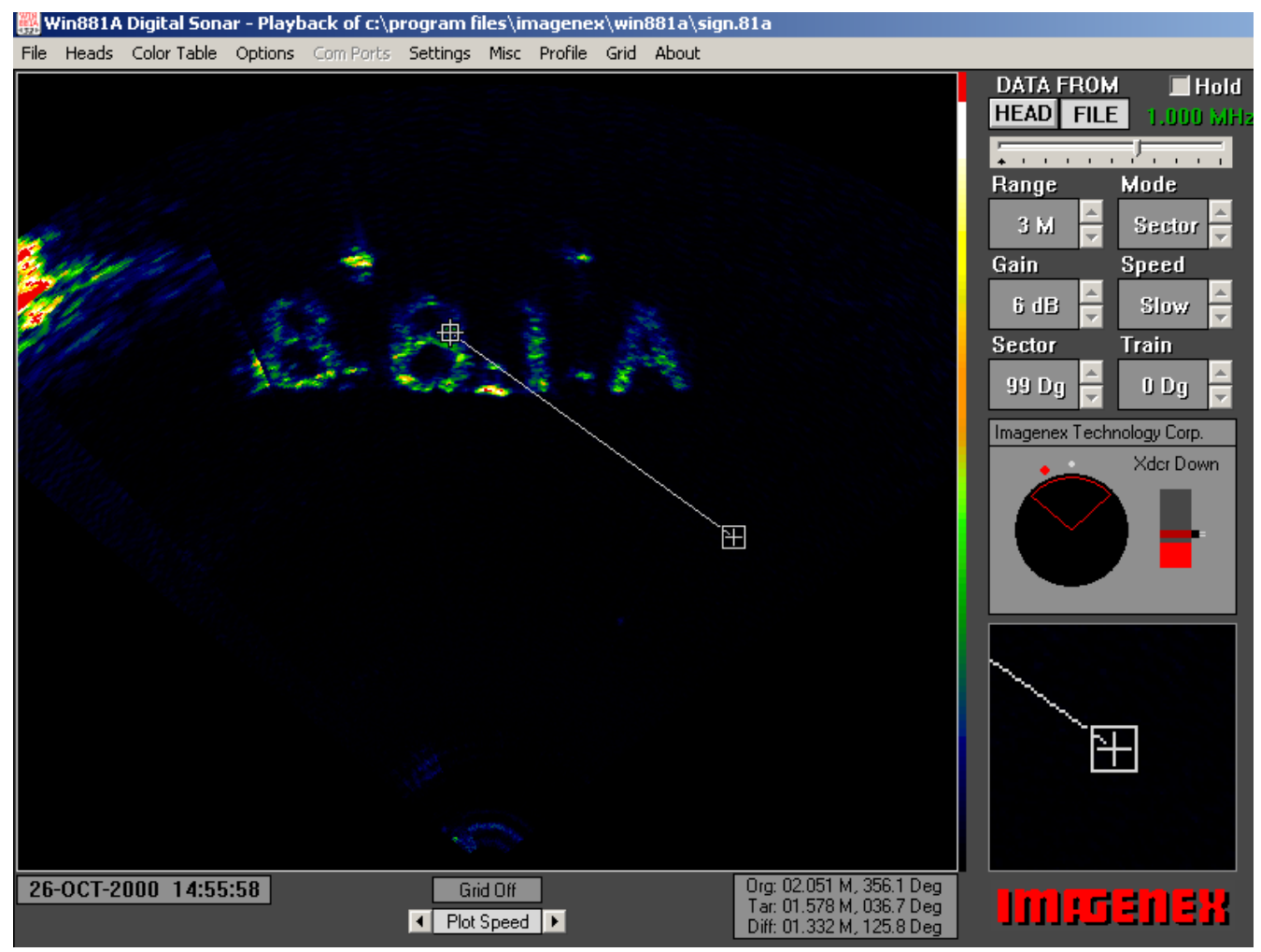

Figure 11: Output Image displaying two-cursor measurement.

Figure 12 shows the table showing the measurement when Double cursor movement is used and also shows the comparison table when this function is not used

\begin{tabular}{|l} 
Org: 02.051 M, 356.1 Deg \\
Tar: 01.741 M, 034.2 Deg \\
Diff: 01.270 M, 118.5 Deg
\end{tabular}

\begin{tabular}{|l|}
\hline Org: 00.000 M, 000.0 Deg \\
Tar: 02.054 M, 353.3 Deg \\
Diff: 02.054 M, 353.3 Deg
\end{tabular}

Figure 12: Figure shows the difference between two cursor and single cursor measurements 


\section{Appendix C}

Code to generate correct sound speed when correct distance are known

Private Sub Command1_Click()

Dim s1, s2, s3, s4, s5 As String

Label8. Visible $=$ True

Text7.Visible $=$ True

Label11. Visible $=$ True

' formula to calculate the correct sound speed

$$
\begin{aligned}
& \text { If } x=1 \text { Then } \\
& i=4921.3 *(p / a) \\
& \text { End If } \\
& \text { If } x=2 \text { Then } \\
& s 1=4921.3 *(p / a) \\
& s 2=4921.3 *(q / b) \\
& i=(s 1+s 2) / 2 \\
& \text { End If } \\
& \text { If } x=3 \text { Then } \\
& \text { s1 }=4921.3 *(p / a) \\
& s 2=4921.3 *(q / b) \\
& s 3=4921.3 *(r / c) \\
& i=(s 1+s 2+s 3) / 3 \\
& \text { End If }
\end{aligned}
$$

$$
\begin{aligned}
& \text { If } \mathrm{x}=4 \text { Then } \\
& \mathrm{s} 1=4921.3 *(\mathrm{p} / \mathrm{a}) \\
& \mathrm{s} 2=4921.3 *(\mathrm{q} / \mathrm{b}) \\
& \mathrm{s} 3=4921.3 *(\mathrm{r} / \mathrm{c}) \\
& \mathrm{s} 4=4921.3 *(1 / \mathrm{d}) \\
& \mathrm{i}=(\mathrm{s} 1+\mathrm{s} 2+\mathrm{s} 3+\mathrm{s} 4) / 4
\end{aligned}
$$

End If

$$
\begin{aligned}
& \text { If } \mathrm{x}=5 \text { Then } \\
& \mathrm{s} 1=4921.3 *(\mathrm{p} / \mathrm{a}) \\
& \mathrm{s} 2=4921.3 *(\mathrm{q} / \mathrm{b}) \\
& \mathrm{s} 3=4921.3 *(\mathrm{r} / \mathrm{c}) \\
& \mathrm{s} 4=4921.3 *(1 / \mathrm{d}) \\
& \mathrm{s} 5=4921.3 *(\mathrm{~m} / \mathrm{e})
\end{aligned}
$$


$\mathrm{i}=(\mathrm{s} 1+\mathrm{s} 2+\mathrm{s} 3+\mathrm{s} 4+\mathrm{s} 5) / 5$

End If

Text7 $=\mathrm{i}$

End Sub

Private Sub Command2_Click()

Form1.Visible $=$ False

Exit Sub

End Sub

Private Sub Form_Load()

'to hide all the labels and text box

Text2. Visible $=$ False

Text3. Visible $=$ False

Text4.Visible $=$ False

Text5. Visible $=$ False

Text6.Visible $=$ False

Text7.Visible $=$ False

Text8.Visible $=$ False

Text9. Visible $=$ False

Text10.Visible $=$ False

Text11.Visible $=$ False

Text12.Visible $=$ False

Label3.Visible $=$ False

Label4.Visible $=$ False

Label5.Visible $=$ False

Label6.Visible $=$ False

Label7.Visible $=$ False

Label8. Visible $=$ False

Labe19. Visible $=$ False

Label10.Visible $=$ False

Label11.Visible $=$ False

End Sub

Private Sub Text1_Change()

' to check the number entered

If Text $1>6$ Then

MsgBox ("PLEASE ENTER ANY NUMBER BETWEEN 1 to 5")

Form1.Refresh

Else 


$$
\begin{aligned}
& \mathrm{x}=\text { Text1 } \\
& \text { Label9. Visible }=\text { True } \\
& \text { Label10. Visible }=\text { True } \\
& \text { End If }
\end{aligned}
$$

' loop to make label and text box visible

If Text $1=1$ Then

Label3. Visible $=$ True

Text2. Visible $=$ True

Text8. Visible $=$ True

End If

If Text $1=2$ Then

Label3. Visible $=$ True

Text2. Visible $=$ True

Text8. Visible $=$ True

Label4. Visible $=$ True

Text3. Visible $=$ True

Text9. Visible $=$ True

End If

If Text $1=3$ Then

Label3. Visible $=$ True

Text2. Visible $=$ True

Text8. Visible $=$ True

Label4. Visible $=$ True

Text3. Visible $=$ True

Text9.Visible $=$ True

Label5.Visible $=$ True

Text4. Visible $=$ True

Text10.Visible $=$ True

End If

If Text $1=4$ Then

Label3. Visible $=$ True

Text2. Visible $=$ True

Text8. Visible $=$ True

Label4. Visible $=$ True

Text3. Visible $=$ True

Text9. Visible $=$ True

Label5. Visible $=$ True

Text4. Visible $=$ True

Text10. Visible $=$ True

Label6.Visible $=$ True

Text5.Visible $=$ True 
Text11. Visible $=$ True

End If

If Text $1=5$ Then

Label3. Visible $=$ True

Text2. Visible $=$ True

Text8. Visible $=$ True

Label4. Visible $=$ True

Text3. Visible $=$ True

Text9. Visible $=$ True

Label5. Visible $=$ True

Text4. Visible $=$ True

Text10.Visible $=$ True

Label6. Visible $=$ True

Text5. Visible $=$ True

Text11.Visible $=$ True

Labe17. Visible $=$ True

Text6. Visible $=$ True

Text12. Visible $=$ True

End If

End Sub

Private Sub Text10_Change()

$\mathrm{c}=$ Text10 'putting the value of text 10 in variable $\mathrm{c}$

End Sub

Private Sub Text11_Change()

$\mathrm{d}=$ Text11 'putting the value of text 1 in variable $\mathrm{d}$

End Sub

Private Sub Text12_Change()

$\mathrm{e}=$ Text12 'putting the value of text 2 in variable $\mathrm{e}$

End Sub

Private Sub Text2_Change()

$\mathrm{p}=$ Text2 'putting the value of text 2 in variable $\mathrm{p}$

End Sub

Private Sub Text3_Change() 
$\mathrm{q}=$ Text3 'putting the value of text 3 in variable $\mathrm{q}$

End Sub

Private Sub Text4_Change()

$\mathrm{r}=$ Text4 'putting the value of text 4 in variable $\mathrm{r}$

End Sub

Private Sub Text5_Change()

$1=$ Text5 'putting the value of text 5 in variable 1

End Sub

Private Sub Text6_Change()

$\mathrm{m}=$ Text6 'putting the value of text 6 in variable $\mathrm{m}$

End Sub

Private Sub Text8_Change()

$\mathrm{a}=$ Text8 'putting the value of text 8 in variable a

End Sub

Private Sub Text9_Change()

$\mathrm{b}=$ Text9

End Sub 'putting the value of text 9 in variable $b$

'Module to declare global variables

Public $\mathrm{p}$ As String

Public q As String

Public r As String

Public 1 As String

Public m As String

Public x As String

Public i As String

Public a As String

Public b As String

Public c As String

Public d As String

Public e As String 